Cahiers Charlevoix

Études franco-ontariennes
Cahiers Charlevoix Études franco-ontariennes

or Crevenerix of

\title{
De la " tradition " à la " participation " : les années 1960 et les mouvements de jeunesse franco-ontariens
}

\section{Michel Bock}

Volume 8, 2010

URI : https://id.erudit.org/iderudit/1039319ar

DOI : https://doi.org/10.7202/1039319ar

Aller au sommaire du numéro

\section{Éditeur(s)}

Société Charlevoix

Presses de l’Université d'Ottawa

ISSN

1203-4371 (imprimé)

2371-6878 (numérique)

Découvrir la revue

Citer cet article

Bock, M. (2010). De la « tradition " à la " participation " : les années 1960 et les mouvements de jeunesse franco-ontariens. Cahiers Charlevoix, 8, 111-196.

https://doi.org/10.7202/1039319ar
Résumé de l'article

Michel Bock continue son étude des mouvements de jeunesse franco-ontariens du milieu du $\mathrm{xx}^{\mathrm{e}}$ siècle. Cette deuxième tranche, qui toise son évolution durant les années 1960, retrace les conflits que cette position engendra. En effet, de nouveaux mouvements de jeunesse (CCJFO, APMJOF et DJ), largement influencés par les valeurs de la contre-culture et l'idéologie " participationniste ", cessèrent de poser le problème franco-ontarien en termes de nation et de nationalité pour adopter, graduellement, la logique d'une identité dont les fondements seraient définis de moins en moins explicitement. Ce renouvellement idéologique se solda par la marginalisation, au nom d'un idéal égalitariste, de la référence nationale canadienne-française, perçue désormais comme trop traditionaliste, élitiste et contraire aux « nouvelles » valeurs de la jeunesse. L'article de Michel Bock analyse le discours, les activités et les relations parfois houleuses qu'entretinrent les quatre principaux mouvements de jeunesse franco-ontariens de 1960 à 1971, période de l'agonie et de la disparition définitive de l'AJFO, que ses concurrentes jugeaient, malgré son évolution, trop peu « représentative » de la jeunesse franco-ontarienne. 


\title{
De la « tradition » à la « participation » : les années 1960 et les mouvements de jeunesse franco-ontariens
}

\author{
Michel Bock \\ Chaire de recherche sur l'histoire de la francophonie canadienne \\ Université d'Ottawa
}




\section{SOMMAIRE}

INTRODUCTION

I - Débats et Remises en CAuse À L'AJfo (1960-1966) 120

A. Former l'élite ou représenter les masses ? 120

B. Identité nationale ou identité personnelle? 125

C. L'AJfo à l'œuvre 134

II - De l'Ajfo À Direction-Jeunesse (1966-1971) 150

A. La Conférence-consultation

de la jeunesse franco-ontarienne (CCJFO) 150

B. L'Association provinciale des mouvements

163

C. Direction-Jeunesse (DJ) 


\section{De la « tradition » à la «participation » : les années 1960 et les mouvements de jeunesse franco-ontariens ${ }^{1}$}

\section{INTRODUCTION}

Pour les mouvements de jeunesse de l'Ontario français, les années 1960 représentent une période d'hésitations, de remises en question et d'interrogations qui se solda par l'évacuation du traditionalisme et de la référence nationale canadienne-française de leur conception de l'identité franco-ontarienne. Certains chercheurs, tels Joseph-Yvon Thériault et Jacques Beauchemin, proposent l'hypothèse que les années 1960 marquèrent le début de la «dénationalisation » de l'identité franco-ontarienne, processus qu'ils définissent comme la liquidation de toute velléité « de se représenter et de s'organiser comme société globale (ce qui est le propre d'une nation), pour se représenter et s'organiser de plus en plus comme une communauté ethnique ou un groupe identitaire $^{2} \gg$. Bien qu'il ne nous appartienne pas, dans le cadre de cette étude, de vérifier cette hypothèse, il est possible, à tout le moins, de retracer la marginalisation de la référence nationale canadienne-française dans l'évolution de la représentation identitaire des mouvements de jeunesse franco-ontariens pendant les années 1960. Sous l'impulsion de facteurs à la fois internes et

1. Nous tenons à remercier le Conseil de recherches en sciences humaines du Canada (CRSH), le Centre interdisciplinaire de recherche sur la citoyenneté et les minorités (CIRCEM) et le Centre de recherche en civilisation canadienne-française (CRCCF) de l'Université d'Ottawa, dont l'appui financier a permis de réaliser cette étude.

2. Joseph-Yvon Thériault, "L'Institution en Ontario français », Mens. Revue d'histoire intellectuelle de l'Amérique française, vol. 6, $\mathrm{n}^{\circ}$ 1, automne 2005, p. 9 ; voir aussi Jacques Beauchemin, «De la nation à l'identité. La dénationalisation de la représentation politique au Canada français et au Québec », dans Simon Langlois et Jocelyn Létourneau (dir.), Aspects de la nouvelle francophonie canadienne, Québec, Presses de 1'Université Laval, 2004, p. 165-187. 
externes, ces derniers cessèrent de poser le problème francoontarien en termes de nation et de nationalité pour adopter, graduellement, la logique d'une identité dont les fondements seraient définis de moins en moins explicitement.

Les mouvements de jeunesse que nous avons retenus pour les fins de cette étude sont au nombre de quatre : l'Association de la jeunesse franco-ontarienne (AJfo, 1949-1971), la Conférence-consultation de la jeunesse franco-ontarienne (CCJFO, 1967-1968), l'Association provinciale des mouvements de jeunes de l'Ontario français (APMJOF, 1968-1971) et Direction-Jeunesse (DJ, 1970-2002). Il s'agit de regroupements dont l'ambition était de parler au nom de la jeunesse franco-ontarienne dans son ensemble et de veiller à la sauvegarde de ses intérêts, intérêts dont la définition évoluerait de manière significative, par ailleurs, durant les années $1960^{3}$. Ont donc été exclues les associations spécialisées comme les mouvements d'Action catholique francoontariens (qui attendent toujours leur historien) et les organismes à portée géographique plus limitée, tels les cercles paroissiaux non affiliés ou encore les nombreux « centres des jeunes » qu'a produits l'Ontario français, surtout en milieu urbain.

Dans une étude antérieure, nous avons analysé les premières années d'existence de l'Association de la jeunesse francoontarienne en soutenant que cette dernière représentait un exemple éloquent de « groulxisme appliqué ${ }^{4}$ ». Nous entendions par là que l'AJFo professait une idéologie conforme aux grandes lignes du nationalisme canadien-français qu'avait défini et véhiculé, entre autres, le chanoine Lionel Groulx. Ce dernier avait proposé une définition traditionaliste de la nation canadienne-française, qu'il concevait comme une communauté de mémoire fondée sur le partage d'une langue, d'une culture et d'une foi, une communauté dont l'existence, qui avait été voulue par la Providence et forgée

3. Les archives de ces associations sont conservées au Centre de recherche en civilisation canadienne-française (dorénavant CRCCF) de l'Université d'Ottawa.

4. Voir Michel Bock, «Un exemple de "groulxisme" appliqué : l'Association de la jeunesse franco-ontarienne de 1949 à $1960 »$, Cahiers Charlevoix, n 7, 2006, p. 277-331. 
par l'Histoire, ne devait rien aux structures politiques ou aux frontières géographiques, auxquelles elle était même antérieure ${ }^{5}$. Des années 1920 aux années 1950, Groulx et son œuvre avaient exercé une influence puissante sur la pensée des milieux nationalistes de tout le Canada français, y compris ceux de l'Ontario avec lesquels il était d'ailleurs étroitement liéc. L'AJfo, au moment de sa fondation en 1949, avait reçu de sa génitrice, l'Association canadienne-française d'éducation d'Ontario (ACFÉO), le mandat de donner aux jeunes « la connaissance exacte de ce qu'ils [étaient] comme [F]ranco-[O]ntariens et la volonté d'accorder leur vie aux exigences de cette personnalité francoontarienne ${ }^{7} \gg$. L'identité que $1^{\prime}$ 'on proposait ainsi aux jeunes ne devait donc pas leur renvoyer le reflet de leur réalité culturelle primaire, de leur condition « anthropologique » pour ainsi dire. Elle avait pour fonction d'infléchir cette réalité, d'affranchir la jeunesse des contingences du quotidien, de lui proposer une manière d'être qui se conformait à une certaine idée du bien commun ou, pour reprendre la belle idée de Fernand Dumont, à un « horizon de la culture ${ }^{8} »$. Le projet national canadien-français auquel souscrivait l'AJFO, durant les premières années de son existence, ne devait pas « refléter », mais « élever », c'est-à-dire « transformer».

Il s'agissait d'un projet pour le moins exigeant. L'AJfo n'était pas, au départ, un club social. Au contraire, ses membres devaient se livrer prioritairement à l'étude nationale et religieuse - ce qui ne les empêchait pas, toutefois, de prendre part à des loisirs « sains » et conformes à leur « personnalité franco-ontarienne».

5. Sur le traditionalisme canadien-français, voir Pierre Trépanier, Qu'est-ce que le traditionalisme? Causerie-débat tenue à Montréal, le samedi 8 juin 2002, [s.1.], Club du 3 juillet, [2002], $53 \mathrm{p}$.

6. Voir Michel Bock, Quand la nation débordait les frontières. Les minorités françaises dans la pensée de Lionel Groulx, Montréal, Hurtubise HMH, 2004, 454 p.

7. [Roger Charbonneau], « Ottawa recevra la jeunesse franco-ontarienne dimanche prochain », [communiqué diffusé à la presse française de l'Ontario le $1^{\text {er }}$ décembre 1949], CRCCF, Fonds Association de la jeunesse franco-ontarienne (dorénavant FAJFO), C9/2/1 (souligné dans l'original).

8. Fernand Dumont, Le Lieu de l'homme. La culture comme distance et mémoire, Montréal, HMH, 1968, 233 p. 
Si la mission de l'Association était d'élever la jeunesse francoontarienne au-dessus des « contraintes » du quotidien, c'était aussi pour former les futurs « chefs » dont la nation canadiennefrançaise aurait besoin, demain, en Ontario. Le traditionalisme de l'Association avait donc comme corollaire une certaine forme d'élitisme que l'on saisissait non pas dans son acception socioéconomique, mais plutôt morale et culturelle ${ }^{9}$. C'était cette élite qui était et qui serait en mesure d'inventer pour le Canada français ce que Benedict Anderson appelle un « imaginaire national ${ }^{10} »$, qui n'est rien d'autre que cet « horizon de la culture » sur lequel les jeunes devaient impérativement mettre le cap. Les années 1960 vinrent cependant bouleverser le nationalisme traditionaliste de l'Ajfo, qui ne s'en remettrait pas. La référence nationale (canadienne-française et catholique) fut progressivement écartée du discours des mouvements de jeunesse au profit d'une définition beaucoup plus floue de l'identité franco-ontarienne. Bien sûr, ce phénomène est partiellement attribuable à des facteurs externes, dont la «québécisation » du discours nationaliste canadienfrançais (bien que les jeunes aient été beaucoup moins prompts à dénoncer le mouvement indépendantiste que leurs aînés ${ }^{11}$ ). Il faut

9. Dans une belle étude de la contribution des intellectuels de droite à la Révolution tranquille, Xavier Gélinas rappelle que, pour eux, l'Homme « universel », c'est-à-dire celui que l'on concevait comme étant libre de toute prédétermination sociale ou culturelle et dont les Lumières avaient souhaité l'avènement, n'était rien d'autre qu'une chimère. L'homme n'était pas «libre » de se recréer entièrement comme s'il n'avait été qu'une page vierge demandant à être noircie. L'homme, au contraire, naissait dans un contexte culturel spécifique qui, sans le contraindre, lui permettait de s'incarner dans la réalité, donc de s'humaniser. Il existait donc, aux yeux des intellectuels de droite, une telle chose qu'un ordre hiérarchique dans la société (et, plus largement, dans la grande société humaine) qui permettait aux divers éléments qui la composaient (individus et groupes sociaux) d'interagir harmonieusement les uns avec les autres. L'existence d'une élite éclairée était donc nécessaire à toute société qui souhaitait progresser normalement et sainement. On constate ici l'influence, encore très profonde, qu'exerçaient sur ces intellectuels le nationalisme organiciste et, depuis le début du $\mathrm{xx}^{\mathrm{e}}$ siècle, le corporatisme social. Voir Xavier Gélinas, La Droite intellectuelle québécoise et la Révolution tranquille, Québec, Presses de l'Université Laval, 2007, $504 \mathrm{p}$.

10. Benedict Anderson, L'Imaginaire national. Réflexions sur l'origine et l'essor du nationalisme, Paris, La Découverte, 1996, 216 p.

11. Sur la position de l'élite nationaliste franco-ontarienne face à la Révolution tranquille et au démantèlement du projet national canadien-français, on consultera Gaétan Gervais, Des gens de résolution. Le passage du Canada français à l'Ontario 
aussi en imputer certains aspects au processus de laïcisation des structures sociales du Canada français, tant au Québec qu'en Ontario, laïcisation qui paraissait même commandée, au moment du concile Vatican II (1962-1965), depuis les plus hautes sphères de la hiérarchie ecclésiastique ${ }^{12}$. À ces deux facteurs, il faut toutefois en ajouter un troisième, encore plus déterminant : l'adhésion croissante des jeunes, dans le contexte de la montée du mouvement contre-culturel, à l'idéologie de participation et aux valeurs égalitaristes, antitraditionalistes et anti-élitistes dont elle était porteuse.

En effet, il n'était pas difficile de croire au « début d'un temps nouveau », pour citer la célèbre chanson de Stéphane Venne interprétée par Renée Claude, un temps qui promettait de conférer à la jeunesse du monde occidental, à la génération de plus en plus bruyante du baby boom, une place centrale. Depuis la fin de la Deuxième Guerre mondiale (1939-1945), l'économie des pays occidentaux avait connu une croissance sans précédent, les gouvernements ne semblaient en avoir que pour le keynésianisme et l'État-providence, alors même que les innovations technologiques foisonnaient et avaient déjà profondément transformé le regard que l'on posait sur le monde et sur ses contraintes matérielles. L'amélioration des conditions de vie et la croissance de la classe moyenne avaient atteint des sommets sans précédent, ce qui entraîna une augmentation non moins spectaculaire de la fréquentation scolaire et universitaire ${ }^{13}$. Dans un tel contexte, l'avenir et ses promesses pouvaient paraître plus agréables à contempler que la «tradition » et les « pesanteurs » du passé. C'est à ce moment, aussi, que les enfants du baby boom, parvenus à l'adolescence et aux premiers jours de leur vie adulte,

Sudbury, Prise de parole, 2003, 230 p. ; Marcel Martel, Le Deuil d'un pays imaginé. Rêves, luttes et déroutes du Canada français, Ottawa, Presses de l'Université d'Ottawa, 1997, $203 \mathrm{p}$.

12. Sur l'impact du concile Vatican II au Canada français, voir Jean Hamelin, Le XXe siècle, tome II : De 1940 à nos jours [dans Nive Voisine (dir.), Histoire du catholicisme québécois], Montréal, Boréal, 1934, 425 p. ; Lucia Ferretti, Brève Histoire de l'Église catholique au Québec, Montréal, Boréal, 1999, 206 p.

13. Doug Owram, Born At the Right Time. A History of the Baby Boom Generation, Toronto, University of Toronto Press, 1996, 392 p. 
investirent l'espace public, forts de leur nombre, de leur assurance et de leur effervescence. Désormais, il suffisait de le désirer, semblait-on croire, pour que la chose se fît. Dans une belle réflexion sur ce qu'il appelle la " génération lyrique », François Ricard estime que, pendant les années 1960 et sous l'impulsion des jeunes du baby boom,

[...] le monde, littéralement, change de régime. Il entre dans un nouvel âge, sous une nouvelle "domination », qui va se répercuter dans l'ensemble de la vie sociale, sinon de la vie tout court. Car la jeunesse, projetant partout ses idées, ses sentiments et ses besoins, les imposera à la communauté tout entière, elle-même rajeunie dès lors, elle-même devenue comme une immense, une unanime jeunesse ${ }^{14}$.

S'agissant du Canada anglais, Doug Owram tient des propos semblables, quoique plus prosaïques, lorsqu'il parle d'une « révolution de la jeunesse ». Nés dans l'abondance matérielle, les jeunes du baby boom étaient parfois plus prompts au matérialisme et à l'hédonisme que leurs aînés qui, eux, avaient connu l'instabilité et les bouleversements de la Crise et de la Guerre $^{15}$; nés au lendemain de l'Holocauste et en pleine guerre froide, ils apprirent à critiquer l'autorité morale dont se prévalait l'élite dirigeante; ayant grandi à l'époque de la multiplication des théories psychologiques sur le bien-être et l'épanouissement personnels, ils cultivèrent l'individualisme et le droit à la différence, voire à l'excentricité : "If society didn't meet [their] needs then society should adjust. [...] If a person or a group [did] not fit into society, then the assumption [was] that society must adjust its perspective ${ }^{16}$. » Autrement dit, la jeunesse exigeait

14. François Ricard, La Génération lyrique. Essai sur la vie et l'œuvre des premiers-nés du baby-boom, Montréal, Boréal, « Boréal Compact », 1994, p. 94.

15. Bien entendu, il faut apporter d'importantes nuances à l'idée du confort matériel dans lequel seraient nés les jeunes du baby boom. Elle n'a de valeur que lorsqu'on la compare à la situation économique beaucoup plus précaire qu'avait connue la génération de leurs parents, qui arrivèrent à l'âge adulte durant la Crise des années 1930 et la Deuxième Guerre mondiale.

16. Doug Owram, Born At the Right Time, op. cit., p. 315. S'agissant cette fois du Québec, Madeleine Gauthier analyse, de son côté, l'apport de la jeunesse - et plus précisément de la jeunesse étudiante - à l'élaboration du « mythe » de la Révolution 
que la société lui renvoyât sa propre image et non qu'elle lui proposât - ou imposât - un modèle identitaire "élitiste » vers lequel elle l'aurait mise au défi de tendre. L'individu - le jeune, en particulier - n'avait de leçon à recevoir de personne. Il devait plutôt chercher à s' " autonomiser » et à construire sa propre identité en toute liberté. Voilà, pourrait-on dire, la logique foncière du mouvement contre-culturel des années 1960, de l'idéologie de participation et de sa critique de l'establishment.

La contestation des jeunes, la mobilisation étudiante, la remise en question de l'ordre établi, la critique de l'élite traditionnelle, de son autoritarisme, de ses valeurs et de ses convictions idéologiques, rien de cela n'était propre à la jeunesse franco-ontarienne, bien entendu. Durant les années 1960, ces phénomènes avaient pris, au contraire, une envergure quasi planétaire. L'Ontario français ne serait pas épargné. En effet, c'est dans un tel contexte de « démocratisation », de « participationnisme » et d'individualisme qu'évoluèrent, durant les années 1960, les mouvements de jeunesse franco-ontariens, qui eurent droit, d'une certaine manière, à leur propre Mai 68. La contestation fut sans doute moins bruyante ici qu'ailleurs dans le monde, mais cela ne l'empêcha pas d'avoir raison du traditionalisme canadien-français que la jeunesse, en une décennie, largua par-dessus bord. Dans les pages qui suivent, nous tenterons de retracer cette évolution idéologique, qui se solda par la marginalisation, au nom d'un idéal égalitariste, de la référence nationale canadienne-française, perçue désormais comme étant trop traditionaliste, élitiste et contraire aux « nouvelles » valeurs de la jeunesse. Nous analyserons le discours, les activités et les relations parfois houleuses qu'entretenaient les quatre principaux mouvements de jeunes franco-ontariens de 1960 à 1971, moment de la disparition définitive de l’AJFO, que ses concurrentes jugeaient, malgré son évolution, trop peu « représentative » de la jeunesse franco-ontarienne.

tranquille (Madeleine Gauthier, « Le Mouvement étudiant des années soixante comme aspect du mythe de la Révolution tranquille ? ", dans Raymond Hudon et Bernard Fournier (dir.), Jeunesse et politique, tome II : Mouvements et engagements depuis les années trente, Sainte-Foy, Presses de l'Université Laval ; Paris, L'Harmattan, 1994, p. 233-255). 


\section{I - Débats et Remises en CAuse À L’AJfo (1960-1966)}

\section{A. Former l'élite ou représenter les masses ?}

La mutation idéologique la plus profonde que subiraient, durant les années 1960, les mouvements de jeunesse franco-ontariens est sans aucun doute leur adhésion progressive à une conception moins élitiste, voire carrément anti-élitiste et égalitariste de l'ordre social, tant et si bien qu'ils rejetteraient jusqu'à la notion même de hiérarchie. Comme nous l'avons déjà expliqué, l' « élitisme » qui avait présidé à la création de l'AJFo n'était pas fondé sur des considérations d'ordre socioéconomique. L'Association avait plutôt la mission de former les futurs chefs de la nation canadienne-française en Ontario, des chefs dont l'autorité serait imputable d'abord et avant tout à leurs qualités morales et intellectuelles. Le culte du chef, de l'homme providentiel qui parviendrait à réveiller la « masse » de sa « torpeur » et à la diriger vers de plus beaux lendemains avait été un thème récurrent dans le nationalisme canadien-français depuis le XIX ${ }^{\mathrm{e}}$ siècle. Il traduisait une certaine conception des relations sociales selon laquelle l'autorité jaillissait non pas du peuple en tant que tel, mais d'une source plus élevée, voire providentielle, sans égard aux régimes politiques qui s'étaient succédé dans l'histoire. Pendant plus d'un siècle, d'ailleurs, des années 1840 aux années 1950, les propagandistes et idéologues nationalistes avaient été nombreux à répéter que le peuple canadien-français était investi d'une mission providentielle, celle de propager la civilisation française et catholique, mission qui justifiait seule son existence et son expansion en Amérique ${ }^{17}$.

Déjà, durant les années 1950, l’AJfo avait dû résoudre la contradiction apparente qui découlait de son double mandat, celui de former des chefs et de regrouper l'ensemble de la jeunesse franco-ontarienne ${ }^{18}$. Elle y était parvenue en postulant que tout

17. Voir Michel Bock, « Se souvenir et oublier : la mémoire du Canada français, hier et aujourd'hui », dans Joseph-Yvon Thériault, Anne Gilbert et Linda Cardinal (dir.), L'Espace francophone en milieu minoritaire au Canada. Nouveaux enjeux, nouvelles mobilisations, Montréal, Éditions Fides, 2008, p. 161-203.

18. Michel Bock, "Un exemple de "groulxisme" appliqué ... », op. cit., p. $277-331$. 
jeune Franco-Ontarien pouvait espérer devenir un chef dans le milieu qui lui était propre, qu'il se fût agi de son environnement de travail, de sa paroisse ou, tout simplement, de son foyer. Durant ses premières années d'existence, toutefois, l'Association était demeurée largement fidèle à sa mission de former la future élite dirigeante du réseau institutionnel de l'Ontario français et avait élevé l'étude nationale et religieuse au premier rang de ses activités.

Après 1960, l'idéal démocratique et le souci de représentativité réaliseraient des incursions de plus en plus profondes dans le discours et les préoccupations de 1'AJFo. L'Association devaitelle continuer à former les futurs chefs nationaux de l'Ontario français, comme elle avait toujours tâché de le faire, ou bien se transformer en un véritable mouvement de masse, question de mieux tenir compte des «besoins » de la jeunesse francoontarienne dans son ensemble ? Dès 1961, le Bureau central de l'AJfo se livra à un douloureux examen de conscience. Depuis environ un an et demi, l'Association avait perdu une douzaine de cercles locaux, de sorte qu'il n'en restait plus, officiellement, que vingt. De ce nombre, seulement quinze fonctionnaient avec un minimum de régularité ${ }^{19}$. La « formule » traditionnelle du cercle paroissial consacré à l'étude nationale et religieuse était-elle épuisée ? L'AJfo devait-elle tenter de ratisser plus large, si elle comptait conserver sa pertinence et franchir les obstacles qui se dressaient sur son passage à l'aube des années 1960 ? Certains étaient de cet avis. En juillet 1961, lors d'une réunion du Bureau central, Jean Léveillé, qui avait été élu à la présidence générale quelques mois auparavant, exposa de la manière suivante l'orientation qu'il souhaitait imprimer au mouvement qui se situait, d'après lui, à la croisée des chemins :

[À] son début, l'AJFo a été conçue par des adultes, pour but adulte $[$ sic]. Nous voyons présentement que l'AJfo doit être

19. Pierre Gravelle, «L'A.J.F.O. actuelle », Le Chaînon, février-mars 1960, p. 7, CRCCF, FAJFO, C9/36/4 ; « Procès-verbal - Réunion régulière du 24 juillet 1961 », p. 1, CRCCF, FAJFO, C9/11/1. 
construite par des jeunes, pour des jeunes, avec des buts qui attirent les jeunes. La jeunesse du vingtième siècle veut tout et elle ne veut rien. Il faut donc lui présenter quelque chose qui saura l'intéresser éminemment. C'est pourquoi le but de la nouvelle formule sera de grouper la jeunesse, et toute la jeunesse. Il ne sera plus question de masse ou d'élite. Il faut grouper la jeunesse et lui infuser avant tout une mentalité française ${ }^{20}$.

Dans cet extrait, ce qui saute aux yeux, c'est le désir de démocratiser les structures du mouvement - et d'en assurer l'indépendance face aux « adultes » (c'est-à-dire l'ACFÉO) -, bien que l'objectif plus traditionnel d' "infuser» aux jeunes une "mentalité française » ne fût pas encore totalement écarté. Léveillé proposa d'amender les statuts de l'AJFO, de façon à autoriser l'admission en son sein non seulement de cercles d'étude, mais aussi de « clubs » pouvant œuvrer dans un nombre élevé de domaines : le sport, la littérature, le divertissement, etc. Bien qu'il ne fût pas question, précisa-t-il, de reléguer l'étude nationale et religieuse aux oubliettes, il n'en était pas moins impératif de « répondre aux appels urgents que lan[çait] toute la jeunesse ${ }^{21} \gg$. Les membres du Bureau central se divisèrent immédiatement en deux clans, celui des « réformateurs » et celui des « traditionalistes », pour ainsi dire. Dans le premier, on répéta que l'AJFo devait « englober toute la jeunesse franco-ontarienne, plutôt qu'une petite minorité, comme elle le fai[sai]t [déjà] ». On souligna que le mot " "étude" fai[sai]t peur à un jeune de 14-15 ans, qui en a[vait] déjà beaucoup de poursuivre ses études scolaires ${ }^{22} »$. Le jeune, au contraire, préférait plutôt « se récréer ». Au lieu de l'austérité d'un cercle d'étude nationale et religieuse, l'AJFo devait plutôt lui offrir un endroit où il pouvait se divertir, tout en développant des habiletés d' « organisateur » qui lui seraient, plus tard, d'une grande utilité lorsque viendrait le temps pour lui de s'engager dans les mouvements adultes. De toute manière, le but non avoué de l'AJFO

20. Ibid., p. 2.

21. Ibid., p. 4.

22. Ibid., p. 4. 
était d'enrayer les mariages mixtes, ce que la souplesse de la nouvelle formule aurait été en mesure de faciliter.

Dans l'autre camp, celui des « traditionalistes », on opposa un refus catégorique à cette interprétation des buts et de l'avenir de l'Ajfo. Le secrétaire général, Michel Gaulin, protesta " énergiquement» et réitéra sa conviction que la mission du mouvement était de fournir à l'individu une «formation intégrale ». En modifiant aussi radicalement la raison d'être et les structures de l'Association, en se lançant frénétiquement dans l'organisation d'activités de tout genre, le danger était grand qu'elle dispersât inutilement ses énergies et, semblait-il dire, qu'elle perdît un peu de son âme. L'heure était plutôt à la consolidation qu'à l'éparpillement ${ }^{23}$. Un autre membre du Bureau central, Jean Gobeil, abonda dans le même sens. La solution au dilemme de l'AJfo se trouvait non pas dans l'abandon de sa mission traditionnelle, mais plutôt dans la persévérance. « Celui qui veut former la jeunesse ne doit pas lui donner ce qu'elle veut $^{24}{ }^{2}$, affirma-t-il sans détour. Que les jeunes préfèrent le jeu à l'étude, rien de plus normal. Ce n'était pas la peine de s'en formaliser : la masse «ne fai[sai]t pas la révolution » et elle ne formait pas un « corps ». Elle suivait, voilà tout, d'où le besoin qu'elle éprouvait d'avoir à sa tête des chefs éclairés et capables de relever les défis du moment.

Les opinions étaient pour le moins tranchées, si bien que les directeurs furent incapables d'en arriver à un commun accord sur le fond des choses. De guerre lasse, manifestement, les « traditionalistes » acceptèrent d'amender les statuts dans le sens de leurs adversaires, mais formulèrent le souhait que le Bureau central eût suffisamment de sagesse pour « établir un programme d'action à la hauteur de la tâche ${ }^{25} \gg$. Cette réunion du Bureau central de l'AJfo revêt une importance toute particulière dans l'histoire du mouvement, puisqu'elle donnerait le ton à l'ensemble des débats qui suivraient sur son orientation générale. En réalité,

23. Ibid., p. 3.

24. Ibid., p. 4.

25. Ibid., p. 4-5. 
l'Association était confrontée à un choix difficile entre deux modes de légitimation radicalement différents : ou bien elle offrirait à la jeunesse ce qu'elle « voulait » et recevrait d'elle ses mots d'ordre, ou bien elle lui offrirait ce dont elle avait « besoin » et continuerait de lui proposer un modèle plus austère d'engagement national qui ne trouverait sans doute jamais preneur qu'auprès d'une minorité, d'une élite. Autrement dit, la légitimité de l'AJfo provenait-elle d'en bas, de la « masse » des jeunes, ou, au contraire, d'en haut, de son élite dirigeante ? Dans quelle mesure était-il nécessaire de choisir entre les deux ?

Chose certaine, le Bureau central déploya, dès lors, de nombreux efforts pour convaincre le congrès général - qui eut lieu trois mois plus tard - d'entériner l'orientation nouvelle qu'il souhaitait imprimer au mouvement. Le nouveau secrétaire général, Pierre Galipeau, fit valoir auprès des membres de l'Association que les nouveaux statuts permettraient à l'Association de combler les « lacunes » du mouvement et d'« unir la jeunesse francoontarienne de toute classe et de tout milieu $^{26} »$. Le thème du congrès était, d'ailleurs, «Unissons-nous ». Dans son mot de bienvenue, le président Léveillé fit de la «refondation » du mouvement le principal enjeu du congrès. L'AJFo devait mieux se « plier» aux « exigences de notre époque » et devenir « plus accessible à la jeunesse franco-ontarienne catholique », toujours sous le «signe », cependant, de la «fierté nationale et du patriotisme ${ }^{27} \gg$. Malheureusement, nous ignorons la suite que donna le congrès général au projet, le procès-verbal des délibérations n'ayant pas été conservé ${ }^{28}$. La discussion n'était pas

26. Lettre circulaire de Pierre Galipeau aux membres de 1'AJfo, 5 septembre 1961, CRCCF, FAJfo, C9/5/5. Michel Gaulin démissionna du secrétariat général le $1^{\text {er }}$ septembre 1961 afin de poursuivre ses études à l'Université de Montréal (lettre de Michel Gaulin aux membres du Bureau central de l'AJfo, 20 août 1961, CrCCF, FAJfo, C9/16/6).

27. Jean Léveillé, mot du président général, programme du huitième Congrès général de l'AJFO, Ottawa, 7-9 octobre 1961, CRCCF, FAJFO, C9/5/6.

28. Les statuts et règlements de l'AJFo en date de décembre 1961 ne font aucune mention de l'existence de « clubs » dans le mouvement. Ils indiquent, tout simplement, que la fonction du cercle est « d'organiser les différentes activités de la jeunesse de son territoire, en conformité avec le but du mouvement » qui, pour sa part, est « de grouper et d'unir la jeunesse franco-ontarienne en vue de développer chez elle une mentalité 
close, toutefois, puisque le Bureau central sentit encore la nécessité de débattre de son orientation dès l'année suivante, en mars 1962 : «L'AJfo est-elle un mouvement d'élite ou un mouvement de masse ? L'AJfo doit-elle tenter de devenir un mouvement dominant tous les autres mouvements de jeunes ${ }^{29}$ ?» se demandat-on, sans qu'une réponse claire fût apportée à la question.

Encore en 1965, l'AJfo tenta, une fois de plus, de ménager la chèvre et le chou et d'offrir " quelque chose aux deux groupes » qui continuaient d'alimenter le « conflit» sur son orientation

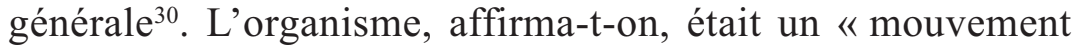
d'élite » en ce sens qu'il offrait un « idéal » à ses membres (bien que cet « idéal» ne fût pas clairement défini). Sa mission était d' " éveiller les jeunes aux problèmes sociaux et nationaux » par l'intermédiaire de l' « étude » et de l'« action ». Si son rôle était de préparer la relève franco-ontarienne à assumer ses responsabilités, il pouvait donc assumer son «élitisme ». Cependant, l'AJfo, qui souhaitait également regrouper l'ensemble de la jeunesse franco-ontarienne, était aussi un "mouvement de masse » : « En effet, c'est dans la masse que se trouvent les chefs que nous voulons former et, partant, l'élite dont nous parlions plus haut ${ }^{31}$. » Et pour l'attirer, cette masse, il ne fallait pas que l'Association limitât ses activités à l'étude.

\section{B. Identité nationale ou identité personnelle ?}

L'AJFo ne rejeta pas d'un seul trait son adhésion au nationalisme canadien-français traditionnel. En fait, ce qui se dégage de ses activités pendant les premières années de la décennie, c'est plutôt la manifestation d'une incertitude idéologique, en même temps qu'un changement graduel de sensibilité, pour ainsi dire, par rapport aux questions touchant à la question nationale. Lors d'une

canadienne-française et catholique par l'étude et par l'action » («Constitution [de l'AJFo] », décembre 1961, p. 1 et 4, CRCCF, FAJFO, C9/1/1).

29. «Association de la jeunesse franco-ontarienne. Réunion spéciale du Bureau central. Le 29 mars 1962 », CRCCF, FAJFO, C9/11/3.

30. «Association de la jeunesse franco-ontarienne. Carnet 1-A. Qu'est-ce que l'AJfo ? », [1966], p. 2, CRCCF, FAJFO, C9-2/1/2.

31. Ibid. 
journée d'étude à Ottawa, en 1962, le président général du mouvement, Louis Vincent, proposa une définition parfaitement traditionaliste de la nation canadienne-française, définition composée des « valeurs » suivantes :

$[\mathrm{U}]$ ne nation est une réunion d'hommes et de femmes ayant une origine et une langue communes ou des intérêts longtemps communs. Il y existe en plus une communauté de sentiments par la communauté même de langue et de religion. Une nation n'est pas nécessairement constituée en [É]tat.

[L] peuple canadien-français est une nation. Il est uni comme tel par sa langue, sa religion, ses coutumes et traditions, sa mentalité, sa culture et ses institutions.

[T]oute nation a le désir normal et naturel de grandir, de se développer et de se répendre [sic].

$[\mathrm{P}]$ our réaliser ce désir, une nation bien organisée doit voir au développement éducationnel, culturel et social des individus la composant. La nation sera forte par la force même des individus.

[P]our ce, il y a donc nécessité de créer des institutions et des associations. Par contre, le mot association se définit comme tel : union de personnes en vue d'un intérêt, d'un but commun.

L'AJFo est ainsi située dans un contexte national ${ }^{32}$.

Interrogés par les participants sur l'importance relative des activités sociales, sportives et intellectuelles à l'AJFO, les membres du Bureau central animant la discussion ${ }^{33}$ répondirent que l'Association était « essentiellement un mouvement national dont le but immédiat [était] de garder la jeunesse, de la former, de lui inculquer ce désir de devenir l'élite de demain ${ }^{34} \gg$.

Cependant, ces convictions seraient bientôt mises à rude épreuve. Si, lors du congrès général de 1963, les participants pouvaient encore écouter le père dominicain Richard Migneault

32. «Journée régionale [Ottawa-Eastview], 23 avril 1962 », p. 1, CRCCF, FAJFO, C9/11/3.

33. Jean Léveillé (ancien président général), Robert de Cotret (premier viceprésident général) et l'abbé René Denis (aumônier général).

34. "Journée régionale [Ottawa-Eastview], 23 avril 1962 », p. 2, CRCCF, FAJFO, C9/11/3. 
disserter sur un thème on ne peut plus orthodoxe, « Ton identité dans la nation », ils assisteraient, le lendemain, à une conférence de Roger Dion, fonctionnaire au ministère de la Santé et du Bienêtre social, traitant un sujet à saveur beaucoup plus individualiste, «Ton identité dans la formation personnelle ${ }^{35}$ ». Le thème du congrès général de 1960 avait été « Deviens un chef ${ }^{36}$ » et s'était encore conformé au traditionalisme des fondateurs de l'Association. En mars 1964, la journée d'étude qu'organisa l'AJFO serait plutôt une exclamation individualiste, majuscules comprises : « $\mathrm{MOI}^{37}$ !». Considérons un autre exemple qui abonde dans le même sens. En 1961, l'AJfo mit sur pied un « cours de chef ». Le responsable du projet était Jean Gobeil, celui-là même qui s'était opposé catégoriquement à la transformation de l'Association en mouvement de masse. Le cours devait se donner en sept séances « par des experts » et comportait deux composantes, orale et écrite ${ }^{38}$. Bien entendu, il s'agissait d'une façon pour l'Association de traduire en gestes concrets sa mission de former les futurs dirigeants nationalistes de l'Ontario français. Manifestement, le caractère élitiste du projet finit par en déranger quelquesuns, puisque trois ans plus tard, en 1964, le « cours de chef» fut transformé en « cours de personnalité sociale ». L'initiative fut conçue, expliqua-t-on, en réponse aux « besoins pressants de la jeunesse franco-ontarienne » et dans le dessein de « définir » le jeune « dans un contexte qui lui [était] propre ${ }^{39} »$. Encore une fois, on aperçoit le caractère de plus en plus hybride du discours identitaire de l'AJFO : si le cours devait encourager les jeunes à développer leur «personnalité », entendue ici comme « ce qui

35. «Association de la jeunesse franco-ontarienne. Neuvième Congrès général, 9, 10 et 11 novembre 1963. Thème : Être ou ne pas être ! ", CRCCF, FAJfo, C9/7/4.

36. Programme du septième congrès général de l'AJFO, 8-10 octobre 1960, CRCCF, FAJFO, C9/4/10.

37. «Association de la jeunesse franco-ontarienne. Journée d'étude, 30 mars $1964 »$, CRCCF, FAJFO, C9/8/1.

38. « Procès-verbal - Réunion régulière du 14 août 1961 [du Bureau central de l'AJfo] », p. 4, CRCCF, FAJFO, C9/11/1. Les documents relatifs au cours de chef de l'AJfo sont contenus dans les dossiers suivants : CRCCF, FAJFO, C9/33, dossiers 10 à 12.

39. «Prospectus. Association de la jeunesse franco-ontarienne. Programme d'étude 1964-[19]65. Cours : "Personnalité sociale" », p. 2, CRCCF, FAJFO, C9/11/8. 
fai[sai]t qu'il[s] [étaient] [eux]-mêmes et [ce] qui le[s] différenci[ait] des autres », il visait aussi à « former des chefs de file compétents $\mathrm{s}^{40} \gg$. On voit bien qu'il subsistait encore quelques traces de l'élitisme traditionnel de l'AJfo dans cette définition de la «personnalité sociale », mais notons, dans le même souffle, que l'objectif était de former non pas des chefs « nationaux », mais bien des chefs « de file », tout simplement. L'évacuation de la référence nationale, qui avait jusqu'ici donné un sens précis à la mission de ces futurs « chefs », était-elle innocente ?

Le texte de la première leçon du cours de personnalité sociale fournit quelques éléments de réponse à cette question. Intitulée « Brise-glace », cette leçon proposait un certain nombre de balises pour aider les jeunes à « comprendre l'individu » ainsi que sa place « dans la société ${ }^{41} »$. Dans un jargon tantôt psychologique, tantôt sociologique, on décrivit abondamment la triple « nature » de la personne (intellectuelle, physique et sociale), les caractéristiques propres à l'adolescence (la confusion et l'incertitude, pour l'essentiel) et les divers agents de socialisation agissant sur la personnalité de l'individu et du jeune en particulier. On y décomposa la société en trois grandes catégories : les « individus », les « grandes organisations » (politiques, religieuses, éducatives, professionnelles, associatives) et, entre les deux, les « groupes médiateurs » (la famille, les amis, le groupe de travail, le cercle de l'AJFo). Dans ce document de dix-huit pages, aucune référence n'est faite à l'appartenance nationale, ni au chapitre de la « nature » de la personne humaine ni parmi les "grandes organisations » qui regroupent, par divers intermédiaires, les individus. Quelques années plus tôt, le programme de l'AJfO comprenait encore des séances d'étude sur les problèmes « nationaux » des Franco-Ontariens, sur la nécessité de faire respecter les droits du peuple canadien-français en Ontario et sur l'œuvre des grands héros de la nation, dont les saints Martyrs

40. Ibid.

41. "Association de la jeunesse franco-ontarienne. Programme d'étude 1964[19]65. Première leçon. "Brise-glace" ", CRCCF, FAJFO, C9/11/8. 
canadiens et Dollard des Ormeaux, pour ne nommer que ceux-là. Les temps avaient bien changé ${ }^{42}$.

Il est impossible de discuter de la marginalisation de la référence nationale canadienne-française dans l'œuvre des mouvements de jeunesse franco-ontariens pendant les années 1960 sans aborder la question du Québec. Le démantèlement du projet national canadien-français, les historiens et les sociologues l'ont bien démontré, est dû en partie aux conséquences politiques, idéologiques et structurelles de la Révolution tranquille. L'AJFO, contrairement à son association mère, l'ACFÉO, consacra assez peu de temps et d'énergie, cependant, à l'analyse de l'impact du phénomène sur l'Ontario français (nous y reviendrons). Comment ne pas voir dans cette indifférence relative un autre signe de la distance croissante que prenait l'AJFO par rapport à la question nationale ? Ses dirigeants étaient pourtant bel et bien conscients de la situation québécoise, qui devait plutôt servir de modèle à la jeunesse franco-ontarienne, comme l'affirmèrent les organisateurs du congrès général de 1965 :

Face à un Québec toujours en ébulition [sic] et envisageant une redéfinition de plus en plus à point du rôle du Canadienfrançais $[s i c]$ dans tout le pays, le congrès étudiera le « leadership » chez les jeunes et chez leurs structures [sic] afin de doter l'AJFo de cet esprit nécessaire à la formation de la jeunesse franco-ontarienne et par conséquent nécessaire à une plus grande ouverture de cette jeunesse sur la société qui les $[$ sic $]$ entoure ${ }^{43}$.

Le thème du congrès était d'ailleurs « Renouveau ». Dans l'esprit des dirigeants de l'AJfo, le « renouveau » dont il était question devait passer par le « réveil à ce que nous sommes » et se manifester dans l'adoption, par les Franco-Ontariens, d'une nouvelle attitude, plus confiante face à l'avenir et affranchie des pesanteurs du passé : «Il ne faut pas avoir peur d'être canadien-français en

42. Mentionnons qu'après 1965, l'AJfo utiliserait les deux appellations en alternance, cours de chef et cours de personnalité, pour désigner le programme.

43. «AJfo. Communiqué. Congrès général prochain de l’AJfo à Ottawa Eastview », [1965], CRCCF, FAJFO, C9/8/5. 
Ontario, parce que minorité ne signifie pas infériorité. Aussi, le terme survivance devrait-il être banni de notre langage. Si les jeunes prenaient leur place dans la société canadienne-française, un monde solide serait bâti où l'on entendrait les mots "vivre", "aller de l'avant", liberté, mais non plus de défense ou de revendication $^{44}$.» Si le concept de « survivance », dans le nationalisme canadien-français traditionnel, avait été doté, le plus souvent, d'une signification romantique et chevaleresque associée à une certaine noblesse, on en réduisit considérablement l'envergure, dans cet extrait, de sorte qu'il ne traduisait plus qu'un complexe d' " infériorité » dont il eût fallu se départir de toute urgence. Il faut également constater, dans ce passage, l'attribution d'une fonction révolutionnaire aux jeunes, présentés comme les dépositaires du progrès, de la liberté et de l'harmonie. Le compte rendu du congrès laisse entrevoir les débats qui avaient cours à l'AjFo depuis le début de la décennie. On y discuta longuement des qualités du chef, de la formation qu'il devait recevoir, ainsi que de ses responsabilités. On reconnut également le rôle essentiel des sociétés adultes, dont il fallait profiter de l'expérience, dans l'orientation générale du mouvement. Si l'Association devait rassembler toute la jeunesse « francophone ${ }^{45}$ » de l'Ontario, elle devait aussi prendre position plus fermement sur les grandes questions de l'heure, dont « la reconnaissance officielle du français en Ontario et la mise sur pied d'un système d'écoles secondaires publiques bilingues (c'est-à-dire françaises $)^{46} \gg$.

Autre signe des temps : c'est durant le congrès de 1965 que les membres de l'AJFo posèrent un geste de rupture déterminant avec le passé de leur mouvement, geste qui alla, cette fois, dans le sens de sa sécularisation. Quatre ans plus tôt, au moment de la révision de ses statuts, l'AJFo ne s'était guère éloignée de la

44. «Association de la jeunesse franco-ontarienne. Dixième congrès général. Rapport préliminaire », [1965], CRCCF, FAJFO, C9/8/6.

45. Bien que nous n'ayons effectué aucune analyse lexicographique du discours identitaire de l'AJFO, il est fort probable qu'il s'agisse là d'une des premières occurrences du terme « francophone » dans les documents de l'organisme.

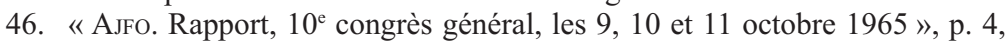
CrCCF, FAJFo, C9/8/6. 
mission nationale et religieuse qui avait été la sienne depuis sa fondation en 1949. On y lit, en effet, que « [1]'Association a[vait] pour but de grouper et d'unir la jeunesse franco-ontarienne en vue de développer chez elle une mentalité canadienne-française et catholique par l'étude et par l'action ${ }^{47} \gg$. Les statuts qu'adopta l'AJfo lors du congrès de 1965 trahissaient la distance qu'elle avait déjà prise par rapport à cette conception de l'identité canadienne-française : «L'[A]ssociation a pour but de grouper et d'unir la jeunesse franco-ontarienne en vue de développer chez elle une mentalité canadienne-française, chrétienne et responsable, un esprit de civisme et de la rendre plus consciente de son héritage culturel, par l'étude et par l'action ${ }^{48}$. » La dimension religieuse ne fut pas complètement écartée, mais de «catholique » qu'elle avait été, l'AJFo était maintenant devenue «chrétienne », tout simplement. Ce changement de vocabulaire et de ton est sans doute partiellement imputable au souci d'œcuménisme et d'aggiornamento qui animait le monde catholique alors que le concile Vatican II tirait à sa fin. L'introduction plus explicite des notions de « responsabilité » et de « civisme » dans la description de la mission de l'AJfo trahissait, elle aussi, ce changement de sensibilité. Les jeunes n'étaient pas que des individus en formation. Ils avaient, d'ores et déjà, des responsabilités à assumer pour le plus grand bien de la société. Mais la sécularisation du mouvement ne s'arrêta pas là. Les statuts de 1965 allèrent jusqu'à éliminer, parmi les critères d'admissibilité de l'Association, l'adhésion de ses membres à la foi catholique (et à toute forme de croyance reli-gieuse, même «chrétienne »). Pour sa part, l'« aumônier», autrefois nommé par «l'autorité religieuse compétente », fut transformé en un simple « conseiller moral nommé par les autorités adultes compétentes ${ }^{49} \gg$. Précisons que

47. «Constitution [de l'AJFo] », décembre 1961, p. 1, CRCCF, FAJFO, C9/1/1.

48. «AJfo. Constitution telle qu'amendée par le dixième congrès général », [1965], p. 1, CRCCF, FAJFO, C9-2/1/1.

49. Michel Bock, «Un exemple de "groulxisme” appliqué... », op. cit., p. 277331 ; «Procès-verbal de la réunion régulière [du Bureau central de l'AJfo] du 17 septembre 1963 », CRCCF, FAJFO, C9/11/6; «Procès-verbal de la réunion régulière du Bureau central de l'AJfo du 24 septembre 1964 », CRCCF, FAJfo, C9/11/8; « Procès- 
l'AJFo était sans aumônier général depuis le départ de l'abbé René Denis en pays de mission à l'été 1963. Malgré les demandes répétées qu'avait adressées le Bureau central à l'archevêché d'Ottawa, le poste de «conseiller moral» ne fut comblé qu'à l'automne 1966 avec la nomination de l'abbé Jean-Claude Proulx. Il semble aussi que quelqu'un, vraisemblablement de l'AcFÉo, ait avisé le Bureau central de demeurer prudent dans cette démarche, si l'on s'en remet au procès-verbal de la réunion du 17 mai 1965 : « On nous conseilla de faire attention et d'attendre qu'on ait un homme sûr à proposer, car un aviseur moral est une arme à deux tranchants : d'un côté, il peut faire beaucoup pour le mouvement; d'autre part, si les choses ne vont pas à son goût, ce même aviseur peut devenir antipathique au mouvement et ainsi nuire au mouvement en ne distinguant pas entre les ordres spirituel et temporel. » Rappelons également que l'abbé Denis avait fait montre d'un conservatisme très rigide lors de son passage au Bureau central de l'AJFo.

L'AJFo était bel et bien consciente de son virage individualiste et de la distance qu'elle avait prise par rapport à ses anciennes convictions nationalistes. À ses yeux, il s'agissait d'une nécessité dictée par le nouveau contexte à l'intérieur duquel évoluait, désormais, la jeunesse franco-ontarienne. Le congrès de 1965 représente un point tournant dans ce sens, le mouvement se faisant dorénavant plus critique que jamais face aux adultes et à toute forme de traditionalisme. Dans un document publié en 1966 et intitulé «Qu'est-ce que l'AJfo ? », la «nouvelle » Association afficha clairement ses couleurs. Le traditionalisme et la modernité ne faisaient pas bon ménage, pas plus que le nationalisme et l'individualisme. Entre la nation et l'individu, la tradition et la modernité, le mouvement avait choisi son camp :

verbal de la réunion du 17 mai 1965 [du Bureau central de l'AJFo] », CRCCF, FAJFo, C9/ $12 / 1$; « Réunion régulière du Bureau central [de l'AJFo] tenue le 22 juin 1966 », CRCCF, FAJFo, C9/12/3 ; « Procès-verbal de l'assemblée générale [du Bureau central de l'AJFo] du 9 août 1966 », CRCCF, FAJFo, C9/12/4 ; l'abbé [Jean-Claude] Proulx, « Message », Nom du bulletin, décembre 1966, p. 17, CRCCF, FAJFO, C9/36/10. 
Si l'AJFo se tourne vers l'aspect formation individuelle, délaissant un peu l'aspect nationaliste, cela provient d'une double nécessité, la compréhension de laquelle conditionne la compréhension de la philosophie actuelle de notre mouvement. À l'heure présente, l'avancement d'un individu dans le monde moderne est non seulement fortement lié à son instruction, mais également à ses qualités personnelles; la complexité des techniques exige l'instruction, tandis que la multiplicité des contacts sociaux humains font $[s i c]$ appel aux qualités que l'individu a su perfectionner. Et l'AJfo est un excellent moyen pour ce faire. La formation individuelle, en plus d'être nécessaire à l'individu pour faire son chemin, l'est aussi pour la société qui a besoin de personnes pour la diriger. La conscience d'appartenir à une société qui a besoin de soi aide grandement l'individu à prendre sa place dans la société et à y assumer ses responsabilité ${ }^{50}$.

Le Bureau central avait débattu de la teneur de ce « carnet », dont la première version avait été carrément rejetée, certains ayant reproché à son auteur, Jean-Louis Renaud, d'y avoir fait « passer le nationalisme avant la personnalité », persuadés qu'une formation «en douce » pouvait « mieux s'effectuer en développant en premier lieu la personnalité du jeune [F]ranco$[\mathrm{O}]$ ntarien $^{51} »$. Dans sa version finale, le carnet se termine presque sur un désaveu du nationalisme et du traditionalisme canadiensfrançais : « L'AJFo n'est pas un endroit où l'on s'attend à écouter sermon sur sermon et discours patriotique sur discours patriotique. » Au contraire, le mouvement visait plutôt « à permettre à l'individu de se former et de se récréer dans une atmosphère qui répond[ît] à son tempérament de chrétien francophone et qui lui perm[ît] de se réaliser et de s'épanouir comme tel ${ }^{52} »$.

50. «Association de la jeunesse franco-ontarienne. Carnet 1-A. Qu'est-ce que l'AJfo ? », [1966], p. 4, CRCCF, FAJFO, C9-2/1/2.

51. «Procès-verbal de l'assemblée générale du 9 août 1966 [du Bureau central de l'AJfo] », CRCCF, FAJfo, C9/12/4.

52. «Association de la jeunesse franco-ontarienne. Carnet 1-A. Qu'est-ce que l'AJfo ? », [1966], p. 3-4, CRCCF, FAJfo, C9-2/1/2. 
Dans le discours de l'AJFo, la « personne » et la « société » étaient en passe de remplacer la « nation ». Son mandat, tel qu'elle l'interpréterait après 1965, était de conduire l'individu francoontarien à assumer ses responsabilités de citoyen dans la société, que l'on présentait parfois - mais de plus en plus rarement comme faisant partie de la nation canadienne-française ${ }^{53}$. Certaines de ces responsabilités étaient à caractère linguistique. L'Association prenait note, en effet, de la gravité du phénomène de l'assimilation, mais la reproduction linguistique n'était plus nécessairement, d'après elle, une nécessité " nationale ». Il s'agissait aussi - et surtout? - d'une nécessité « pratique », qui n'exigeait plus la conscience de partager, collectivement, une " référence », dans le sens dumontien du concept, se déployant dans le temps et transcendant les contingences du quotidien ${ }^{54}$. « Il y a donc une nécessité, expliquait l'AJFo, de démontrer au jeune que l'utilisation de sa langue ne représente pas seulement un atout culturel, mais peut être utile d'une façon pratique et immédiate. Ce n'est [pas] par fierté nationale seulement que les [F]ranco-[O]ntariens doivent parler le français ; la société ellemême doit leur fournir cette motivation. L'AJfo peut devenir un facteur intermédiaire de la société pour fournir cette motivation $^{55}$. »

\section{L'AJfo à l'œuvre}

Les activités de l'AJFo, entre 1961 et 1966, reflètent la diversification et la multiplication de ses intérêts et de ses priorités, en même temps que la distance qu'elle avait prise vis-à-vis de la question nationale. Ses dirigeants, répétons-le, n'écartèrent pas du jour au lendemain la dimension strictement nationaliste de leurs préoccupations. L'organisme, par exemple, déploya de

53. «Association de la jeunesse franco-ontarienne. Carnet 1-B. Les buts l'AJFo ? », [1966], p. 1, CRCCF, FAJFO, C9-2/1/2.

54. Fernand Dumont, Genèse de la société québécoise, Montréal, Boréal, 1996, p. $342-348$.

55. «Association de la jeunesse franco-ontarienne. Carnet 1-A. Qu'est-ce que l'AJfo ? », [1966], p. 1, CRCCF, FAJFO, C9-2/1/2. 
nombreux efforts pour faire respecter les droits de la langue française dans les instances publiques fédérales et provinciales ${ }^{56}$, pour favoriser l'expansion de la radio et de la télévision françaises en Ontario (en particulier dans le Sud) ${ }^{57}$, en même temps qu'elle continua de nouer des liens avec les autres minorités canadiennesfrançaises du pays, soit en participant à leurs activités, soit en accueillant à Ottawa des délégations provenant de leurs propres mouvements de jeunesse ${ }^{58}$. Dans ce dernier cas, précisons que les initiatives de l'AJFo étaient devenues plutôt ponctuelles et circonstancielles depuis la disparition du Conseil national de la jeunesse canadienne-française ( $\mathrm{C} \mathrm{NJCF})$, organisme qu'elle avait fondé en 1958 avec l'Association de la jeunesse canadienne du Québec (AJC) et la Relève albertaine ${ }^{59}$. Il semble que le CNJCF n'ait plus existé que sur papier après 1960. L'AJfo prétexta le

56. Voir, par exemple : lettres de Jean Léveillé à John Diefenbaker, premier ministre du Canada, 20 mars 1961 et 17 octobre 1961, CRCCF, FAJFO, C9/16/5 et C9/17/1 ; lettre de Jean Léveillé à la Commission du service civil, 10 avril 1961, CRCCF, FAJFO, C9/16/5 ; «Procès-verbal - Réunion régulière du 14 avril 1965 [du Bureau central de l'AJFO] », CRCCF, FAJFO, C9/10/15 ; lettre de Michel Gaulin à H. L. Rowntree, ministre des Transports de l'Ontario, 22 août 1961, CRCCF, FAJFO, C9/16/6 ; " Procèsverbal de la réunion régulière du 29 décembre 1963 [du Bureau central de l'AJFo]", CrCCF, FAJfo, C9/11/6; «Procès-verbal de la réunion régulière [du Bureau central de l'AJfo] tenue le 16 avril $1966 »$, CRCCF, FAJFo, C9/12/3.

57. Voir, par exemple : lettre de Rémy Beauregard à W. D. Mills, secrétaire du Bureau des gouverneurs de la Radiodiffusion, 15 janvier 1963, CRCCF, FAJFO, C9/17/ 6 ; lettre de Rémy Beauregard aux membres du Bureau de direction de Radio-Canada (Montréal), 29 mai 1963, CRCCF, FAJFO, C9/18/1 ; lettre de Georges Bellefleur, président du sous-comité des finances du Comité de Radio-Télévision française de la péninsule du Niagara, s.d., CRCCF, FAJFO, C9/18/1 ; lettre de Mario Lalanne, publiciste, Association de radio-télévision française du Sud de l'Ontario, à Louis Vincent, 8 juillet 1963, CRCCF, FAJFO, C9/41/2 ; " Procès-verbal de la réunion régulière du 15 juillet 1963 », CRCCF, FAJFO, C9/11/6 ; « Procès-verbal de la réunion régulière tenue le 24 mars 1966 », CRCCF, FAJFO, C $9 / 12 / 3$.

58. Voir, par exemple : lettre de Maurice Lavallée, Association canadiennefrançaise de l'Alberta, à Michel Gaulin, 24 avril 1961, CRCCF, FAJFO, C9/16/5 ; « Procèsverbal - Réunion régulière du $1^{\text {er }}$ septembre 1961 [du Bureau central de l'AJFo] », CRCCF, FAJFO, C9/11/1 ; «Procès-verbal - Réunion régulière du 25 septembre 1961 [du Bureau central de l'AJFO] », CRCCF, FAJFO, C9/11/2; « Procès-verbal de la réunion régulière du 17 juin 1964 [du Bureau central de l'AJfo] », CRCCF, FAJFo, C9/11/7 ; « Procès-verbal de la réunion du 29 juillet 1965 [du Bureau central de l'AJfo] », CRCCF, FAJfo, C9/12/1 ; Pierre Allard, « Les Francos se rencontrent - L'Alberta en Ontario », Nom du bulletin, juin 1966, p. 14-15, CRCCF, FAJFO, C9/36/10.

59. Voir Michel Bock, "Un exemple de "groulxisme" appliqué... », op. cit., p. $277-331$. 
manque de financement pour expliquer l'oubli dans lequel l'organisme pancanadien était tombé (sans compter que la Relève albertaine paraissait avoir elle aussi du plomb dans l'aile $)^{60}$, mais il y a fort à parier que la désaffection de l'AJc n'était pas étrangère au problème, elle dont l'AJFo avait déjà déploré la faiblesse du sentiment national à l'aube de la Révolution tranquille ${ }^{61}$.

C'est d'ailleurs au début des années 1960 que les critiques de l'AJfo furent les plus nombreuses vis-à-vis de la redéfinition, au Québec, de la doctrine nationaliste traditionnelle. En juillet 1961, une rencontre au Québec avec le directeur des Équipiers de SaintMichel, l'abbé Jean-Paul Tremblay, le président de l'Asc, Paul Simard, et le Frère Untel, pseudonyme du frère mariste JeanPaul Desbiens, fit au Bureau central une « mauvaise impression » en raison, notamment, de leurs idées « difficiles à définir » sur le nationalisme, l'internationalisme et les mouvements de jeunesse ${ }^{62}$. La décision fut alors prise de n'envoyer qu'un seul délégué à la « Semaine de fraternité française » qu'organisaient les Équipiers de Saint-Michel la semaine suivante, soit Pierre Galipeau. Ce dernier revint de la rencontre fort déçu, à la fois du nombre peu élevé de participants et de la place, « à peu près nulle », qui avait été faite durant la rencontre à l'étude nationale ${ }^{63}$.

Par rapport au mouvement indépendantiste comme tel, l'AJFO n'adopta cependant pas de position très claire. En 1961, elle projeta de mener une enquête sur la question, mais il semble qu'elle n'ait pas donné suite au projet ${ }^{64}$. Lorsque l'AcFéo se prononça sur la question du « séparatisme » en faisant montre de

60. «Procès-verbal - Réunion régulière du $1^{\text {er }}$ mars 1962 [du Bureau central de l'AJFO] », CRCCF, FAJFo, C9/11/3 ; lettre de Léonard Fournier, Association canadiennefrançaise de l'Alberta, 11 février 1963, « Procès-verbal - Réunion régulière du 14 août 1961 [du Bureau central de l'AJfo] », CRCCF, FAJfo, C9/17/6.

61. «Procès-verbal - Réunion régulière du 14 novembre 1960 [du Bureau central de l'AJFo] », CRCCF, FAJFO, C9/10/14.

62. «Procès-verbal - Réunion régulière du 4 juillet 1961 [du Bureau central de l'AJfo] », CRCCF, FAJFO, C9/11/1.

63. «Procès-verbal - Réunion régulière du 14 août 1961 [du Bureau central de l'AJfo] », CRCCF, FAJfo, C9/11/1.

64. «Procès-verbal de la réunion régulière du 30 novembre 1961 [du Bureau central de l'AJFo] », CRCCF, FAJFo, C9/11/2. 
sympathie envers le principe de la chose, mais en réitérant sa foi en l'avenir de la Confédération, l'Ajfo se contenta, tout simplement, d'en prendre note ${ }^{65}$. L'Association comptait aussi soumettre un mémoire à la Commission royale d'enquête sur le bilinguisme et le biculturalisme, mais le surmenage l'aurait empêchée de mettre le projet à exécution ${ }^{66}$. Elle trouva le temps, tout de même, de participer aux États généraux du Canada français. Dans le «schéma de mémoire » qu'elle présenta à l'ACFÉo le 5 octobre 1966, soit un mois et demi avant la tenue des assises préliminaires à Montréal, elle constata que le Canada français, « [d] epuis quelque temps déjà, cherch[ait] à se définir » et à rattraper le retard qu'il accusait sur le reste du pays en matière d' « économie », de « technique », d' " éducation », de « sécurité sociale » et de « politique ${ }^{67} »$. En revanche, le seul domaine dans lequel les gains avaient été moins substantiels était le domaine constitutionnel. Rejetant d'emblée aussi bien la centralisation fédérale que la solution indépendantiste, l'AJfo opta pour l'octroi au Québec d'un statut particulier qui en aurait fait un État associé à l'intérieur de la fédération canadienne : « L'option d'État associé permettrait aussi au Québec de se gérer d'une façon souveraine et de répondre plus facilement aux besoins de la nation canadienne-française ». L'AJFo enchaîna, toutefois, en affirmant

65. «Déclaration de l'Association canadienne-française d'éducation d'Ontario sur le mouvement séparatiste », 11 décembre 1961, CRCCF, FAJFO, C9/40/5 ; « Procèsverbal - Réunion régulière du 22 décembre 1961 [du Bureau central de l'AJfo] », CRCCF, FAJFO, C9/11/2.

66. «Procès-verbal de la réunion du 29 juillet 1965 [du Bureau central de l'AJfo] », CRCCF, FaJfo, C9/12/1. Dans le plan du mémoire, qui fut toutefois conservé, on affirma que « le besoin des deux langues [était] essentiel et [que] la participation égale des deux peuples à l'œuvre commune [était] un principe de base qui favorisera[it] l'épanouissement normal du Canada », mais qu'il n'[était] pas nécessaire de définir les mots « culture », « bilinguisme » et « biculturalisme », que cela n'aurait représenté qu'une perte de temps pouvant porter à confusion. On projeta aussi de « commenter le multiculturalisme en admettant l'apport des autres cultures » et on s'interrogea sur ce qui devait être fait pour les «premiers Canadiens », c'est-à-dire les populations autochtones (« Mémoire à [la] [C]ommission royale d'enquête sur le bilinguisme et le biculturalisme », CRCCF, FAJFO, C9/11/8).

67. « Schéma du mémoire à être présenté par la délégation ontarienne aux États généraux du Canada français. Préparé par : l'Association de la jeunesse francoontarienne », 5 octobre 1966, CRCCF, Fonds Association canadienne-française de l'Ontario, C2/355/5. 
qu'il fallait que toute révision constitutionnelle tînt compte du fait fondamental que « la nation canadienne-française ne se limit[ait] pas au Québec » et que les minorités françaises devaient bénéficier de la même reconnaissance et des mêmes droits que ceux qui, historiquement, avaient été accordés à la minorité angloquébécoise. Tout en plaidant, en conclusion, pour que la « société assur[ât] à la jeunesse la possibilité de vivre selon sa mentalité et sa culture », l'Association réitéra sa foi dans un des éléments centraux du credo nationaliste traditionnel, soit que le Québec, dont les minorités françaises dépendaient pour assurer leur développement, continuât de les considérer, en revanche, « comme le prolongement de la nation canadienne-française » ou encore comme des « zones tampons pour la survie du Canada français ».

L'on constate aisément, encore une fois, que la référence nationale canadienne-française, en 1966, n'avait pas été entièrement écartée de la conception que se faisait l'AJFo de l'identité de la jeunesse franco-ontarienne. Ce mémoire se conformait encore à plusieurs aspects du nationalisme canadien-français traditionnel - référence religieuse en moins, ce qui est toutefois loin d'être insignifiant. Il faut néanmoins reconnaître que l'AJFO semblait nettement moins hostile au mouvement indépendantiste et à la «québécisation » du discours nationaliste que ne le deviendrait l'ACFÉO, si l'on peut en tenir pour preuve sa proposition d'accorder au Québec le statut d'État associé à l'intérieur de la fédération canadienne ${ }^{68}$. Au retour des assises préliminaires de 1966, les quatre délégués de l'Ajfo se dirent relativement satisfaits de la tournure des événements ${ }^{69}$. Manifestement, ils

68. Pour une analyse exhaustive de la participation des Franco-Ontariens et de l'AcFÉo aux États généraux, on lira Gaétan Gervais, «L'Ontario français et les "États généraux du Canada français" (1966-1969) », Cahiers Charlevoix, n 3, 1998, p. 231-364.

69. Il s'agissait de Jules Labelle, de Jean-Louis Renaud, de Réjeanne Beauregard et du conseiller moral général, l'abbé Jean-Claude Proulx (« Procès-verbal de l'Assemblée générale du Bureau central [de l'AJFo], le 17 novembre 1966 », CRCCF, FAJFO, C9/12/4; Les États généraux du Canada français. Assises préliminaires tenues à l'Université de Montréal, du 25 au 27 novembre 1966, [s.1.], [s.é.], 1966, p. 121-122). 
avaient craint le pire, c'est-à-dire que les représentants « d'outrefrontières » fussent noyés parmi les délégués du Québec, beaucoup plus nombreux. L'option indépendantiste avait été contenue, estimèrent-ils, et « on s'aperçu[t] d'une présence réelle de la nation canadienne[-] française, surtout des minorités ${ }^{70} \gg$. Aux assises de 1967, l'AJFo dépêcha cette fois cinq délégués ${ }^{71}$. Les procès-verbaux de l'Association ne font malheureusement pas état de leur passage à Montréal. Nous savons, cependant, que l'un deux, Jean-Louis Renaud, prit la parole lors du débat sur l'adoption de la « déclaration préliminaire », qui affirmait que les « Canadiens-Français [sic] constitu[ai]ent une nation », que « le Québec constitu[ait] le territoire national et le milieu politique fondamental de cette nation » et que « la nation canadiennefrançaise a[vait] le droit de disposer d'elle-même et de choisir librement le régime politique sous lequel elle entend[ait] vivre ${ }^{72} »$. Cette déclaration de principes, qui était destinée à donner le ton à l'ensemble des délibérations, contribua à soulever la colère de l'ACFÉO, entre autres, qui déciderait de se retirer des assises de 1969. Renaud, pour sa part, affirma que les jeunes FrancoOntariens - dont il se faisait le « porte-parole »- appuyaient la proposition, mais voulut y apporter, dans le même souffle, un certain nombre d'amendements. Si le Québec avait le droit de « disposer de lui-même » et de « choisir librement le régime politique sous lequel il entend[ait] vivre », il fallait préciser, d'une part, que les minorités ne possédaient pas de telles «potentialités ». D'autre part, Renaud souhaitait que l'assemblée ajoutât un élément à la déclaration qui eût précisé que « [1]es CanadiensFrançais [sic] établis ailleurs au pays [devaient] se voir garantir les droits essentiels à leur épanouissement collectif aussi bien

70. « Procès-verbal de l'assemblée du Bureau central [de l'AJfo] du 15 décembre $1966 »$, CRCCF, FAJFO, C9/12/4.

71. «Procès-verbal, assemblée régulière du Bureau central [de l'Asfo], le 16 août 1966 ", CRCCF, FAJFo, C9/12/5; Les États généraux du Canada français. Assises nationales tenues à la Place des Arts de Montréal du 23 au 26 novembre 1967, [s.1.], [s.é.], 1967, p. 349-350.

72. Ibid., p. 42. 
qu'individuel ${ }^{73} \gg$. L'assemblée rejeta ces propositions d'amendement, ce qui n'empêcha pas les représentants de la jeunesse francoontarienne d'appuyer la résolution, tranchant ainsi avec l'ensemble de la délégation franco-ontarienne, qui la rejeta à raison de $55 \%{ }^{74}$.

La tolérance relative que semblait manifester l'AJFo à l'endroit de l'indépendantisme québécois découlait-elle de la distance qu'elle avait elle-même commencé à prendre vis-à-vis du nationalisme canadien-français traditionnel ? L'hypothèse nous paraît séduisante. Chose certaine, au fur et à mesure que progressait la décennie, la question nationale balisait de moins en moins la réflexion de l'Association sur l'identité de la jeunesse franco-ontarienne. L'objectif du « cours de personnalité » dont nous avons présenté les grandes lignes ci-dessus était de faire découvrir au jeune la vraie «nature » de l'individu, que l'on présentait comme étant composée de trois dimensions, intellectuelle, sociale et physique. À une autre époque, l'AJFO n'aurait sans doute relevé, pour l'essentiel, que deux dimensions constitutives de la «nature » humaine, soit le national et le religieux. Cette conception de l'individu et de sa place dans la société révèle la distance croissante qui s'interposait désormais entre l'Association et ses convictions nationalistes et traditionalistes. De la triple nature de l'individu découlaient autant de besoins particuliers qu'il lui fallait combler, croyait-elle désormais. Un programme fondé uniquement - ou, à tout le moins, prioritairement - sur l'étude nationale et religieuse ne suffisait plus, puisque l'AJfo ne devait plus limiter le champ de ses activités aux seuls besoins « intellectuels » des jeunes. Les dirigeants de l'Association en vinrent à croire qu'une formation véritablement intégrale devait aussi tenir compte des éléments social et physique de leur personnalité, qui étaient mis sur un pied d'égalité avec

73. Ibid., p. 45-46.

74. Fernan Carrière, « La Métamorphose de la communauté franco-ontarienne, 1960-1985 », dans Cornelius Jaenen (dir.), Les Franco-Ontariens, Ottawa, Presses de l'Université d'Ottawa, 1993, p. 321 ; Les États généraux du Canada français. Assises nationales tenues à la Place des Arts de Montréal du 23 au 26 novembre 1967, p. 49. 
l'élément intellectuel. C'est à une véritable réhabilitation des loisirs et des activités récréatives que l'on assistait, peu à peu, non seulement dans le discours de l'AJFo, mais aussi dans son œuvre.

Les loisirs occupèrent, en effet, une place de plus en plus proéminente dans la programmation de l'Association, et ce, dès le début de la décennie ${ }^{75}$. En 1963, par exemple, l'AJfo s'engagea - sous l'impulsion de l'AcFÉo, précisons-le - à effectuer une vaste enquête sur les loisirs, d'abord à Ottawa, ensuite à l'échelle de la province $^{76}$. Armée d'un questionnaire qu'elle fit circuler dans la capitale fédérale parmi ses membres, l'Association préleva de nombreux renseignements sur leurs habitudes de lecture, la nature de leurs sorties à caractère social et culturel, le temps qu'ils consacraient à la télévision, aux jeux, à la danse et aux sports, ainsi que la langue dans laquelle se déroulait l'ensemble de ces activités récréatives ${ }^{77}$. Après avoir effectué plus de 500 appels téléphoniques et « un nombre incalculable d'entrevues », l'AJFO présenta, en juin 1963, un « Inventaire des loisirs dans l'Ontario français » qui brossa un portrait plutôt sombre des activités récréatives de la jeunesse franco-ontarienne, du moins en ce qui avait trait à la dimension linguistique :

Nous concluons que, à l'exception de la télévision française qui est regardée par un petit pourcentage de jeunes, et à l'exception des milieux universitaires et scolaires où le français trouve une plus grande place, les loisirs des jeunes de l'Ontario se déroulent dans une ambiance presque totalement anglaise.

Nous soulignons que la musique et le vocabulaire sont les deux facteurs qui influent le plus sur l'ambiance de tout loisir. Nous relevons qu'une grande proportion des jeunes communiquent entre eux en anglais de façon habituelle ${ }^{78}$.

75. «Association de la jeunesse franco-ontarienne. Bureau central. Le Secrétariat. Procès-verbal de la réunion spéciale du 26 septembre 1962 », CRCCF, FAJFO, C9/11/4.

76. " Procès-verbal de la réunion spéciale [du Bureau central de l'AJfo] du 24 février $1963 »$, CRCCF, FAJFO, C9/11/5.

77. Le Bureau central, lettre circulaire et questionnaire de l'enquête sur les loisirs, 27 février 1963, CRCCF, FAJFO, C9/17/6.

78. «Inventaire des loisirs dans l'Ontario français, juin 1963 », CRCCF, FAJFO, $\mathrm{C} 9 / 7 / 5$. 
L'AJfo n'avait encore jamais établi de lien aussi direct entre la marginalité des activités récréatives de langue française et la menace de l'assimilation qui pesait sur les Franco-Ontariens et, en particulier, sur les jeunes. On précisa, également, que l'interdiction qu'avaient décrétée les autorités ecclésiastiques dans certains milieux franco-ontariens sur l'organisation d'activités sociales mixtes, telles les soirées dansantes, n'avait eu d'autre conséquence que de pousser les jeunes vers des boîtes de nuit où l'ambiance, encore une fois, était très largement anglaise. Dans le cas de l'AJFO, la mise au rancart progressive de l'étude nationale et religieuse à la faveur d'une programmation davantage axée sur les loisirs n'était donc pas tout simplement la traduction d'un quelconque virage hédoniste. Il en allait carrément, semblait-on dire, du combat contre l'assimilation. Il est à noter, par ailleurs, que les auteurs du document ne posèrent aucun jugement sur la nature comme telle des activités auxquelles se livraient les jeunes FrancoOntariens, au-delà de la question linguistique. Dix ans plus tôt, l'AJFo avait dénoncé les effets pernicieux de la culture populaire américaine, dont la musique rock-and-roll et le cinéma hollywoodien, et avait plutôt cherché à promouvoir l'organisation de loisirs « sains », « formateurs » et en accord avec le caractère « national » des Franco-Ontariens ${ }^{79}$. Il n'avait pas suffi, autrement dit, de déplorer tout simplement que ces activités se déroulassent uniquement en anglais : c'était l'ensemble des valeurs que véhiculait la culture américaine, jugées contraires à la culture nationale des Canadiens français, qui avait excité la méfiance des dirigeants de l'Association. Pendant les années 1960, toutefois, la position de l'AJFO, là-dessus, n'était déjà plus tout à fait la même. Si l'on en tient pour preuve les résultats de cette enquête sur les loisirs, on ne considérait plus le rock-and-roll et la musique populaire américaine, par exemple, comme étant pernicieux en soi, comme étant porteurs de valeurs culturelles et nationales contraires à celles des Franco-Ontariens. Il aurait suffi,

79. Voir Michel Bock, «Un exemple de "groulxisme” appliqué... », op. cit., p. 300-304. 
semblait-on dire, que la jeunesse franco-ontarienne pût accéder à cette culture dans sa propre langue, tout simplement.

Quoi qu'il en fût, l'AJFo en vint progressivement à la conclusion que ce qu'il y avait de mieux à faire pour rendre service et « plaire » aux jeunes Franco-Ontariens, c'était de leur offrir le moyen de se divertir et de se récréer en français. Lorsque le viceprésident de l'Association, Gilles Blache, demanda à l'AJfo de l'appuyer dans ses démarches visant à mettre sur pied une boîte de nuit « à caractère français » à Ottawa, ses collègues du Bureau central l'appuyèrent sans réserve ${ }^{80}$. Le «Bistro », tel qu'on le baptisa, ouvrit ses portes à l'été 1963 et proposait aux jeunes, tous les jeudis, « un endroit où ils pou[vaie]nt se divertir en français $^{81} »$. Le soir de l'ouverture officielle, le populaire Paolo Noël accepta même de monter sur les planches du Bistro et d'y faire un tour de chant. Le Bistro était-il, cependant, une créature de l'AJfo ou une initiative privée et individuelle de son viceprésident, Gilles Blache ? Les relations entre le Bistro et l'Association semblent avoir été pour le moins ambiguës, en effet. Officiellement, ils demeuraient distincts l'un de l'autre et conservaient leur entière indépendance. Dans les faits, le secrétariat de l'AJfo était aussi celui du Bistro, de sorte que, sur le plan administratif, la confusion était grande ${ }^{82}$. Par ailleurs, le président général, Louis Vincent, annonça, à l'automne 1963, que le défunt Conseil national de la jeunesse canadienne-française (CNJCF) allait renaître de ses cendres et prendre sous sa tutelle la gestion du Bistro ${ }^{83}$. En réalité, le C $\mathrm{NJCF}$ serait rapidement rebaptisé le «Syndicat national des œuvres de la jeunesse » (SYNOJ) et n'aurait plus rien à voir avec l'organisme qui, pour un temps, avait ambitionné d'unir la jeunesse nationaliste de tout le Canada

80. « Procès-verbal de la réunion régulière [du Bureau central] du 18 mars 1963 », CRCCF, FAJFO, C9/11/5.

81. Lettre circulaire de Rémy Beauregard, 10 juin 1963, CRCCF, FAJFO, C9/18/1.

82. Lettre de Rémy Beauregard à G.-H. Dagneau, directeur, Service du Canada d'outre-frontières, ministère des Affaires culturelles du Québec, 27 novembre 1963, CRCCF, FAJFo, C9/18/2.

83. « Procès-verbal de la réunion régulière [du Bureau central] du 10 septembre 1963 », CRCCF, FAJfo, C9/11/6. 
français. L'été suivant, le Bureau central irait encore plus loin en faisant du SYNoJ un organisme-parapluie regroupant à la fois l'AJfo, le Bistro et le « Coin du livre », vraisemblablement une librairie sur laquelle les archives de l'AJFo sont malheureusement peu bavardes ${ }^{84}$.

À l'automne 1964, la question des rapports de l'AJFo avec le SynoJ servirait de prétexte à un nouvel affrontement entre « traditionalistes » et " réformateurs » sur l'orientation générale du mouvement. L'Association, manifestement, cherchait sa voie et éprouvait toujours beaucoup de mal à trancher entre les loisirs et l'étude, entre la masse et l'élite. Chose certaine, les critiques dardaient de toutes parts. Au moment d'organiser un « ralliement » de jeunes à North-Bay, en octobre 1964, le Bureau central reçut une missive du père montfortain Onil Perrier, vicaire de la paroisse Saint-Vincent-de-Paul et aumônier du cercle Racine de NorthBay, demandant que l'Association organisât surtout des activités récréatives dans le cadre de l'événement, afin de stimuler l'intérêt des jeunes de la région et d'augmenter leur participation :

[L]'AJFo réussirait beaucoup mieux si elle s'appliquait à former [...] les jeunes dans des stages spéciaux (camps ou autres genres). Je me rends compte aussi que même du point de vue national ou patriotique, ce que fait le P. Régimbal [sic] [du Centre des jeunes de Sudbury] est plus pratique, i.e. nos jeunes n'étant pas assez français de cœur, on ne peut les grouper autour d'un idéal patriotique; mais par contre, si on les unit ainsi dans une expérience profonde de fraternisation humaine par les loisirs, avec des chants et des discussions en français pendant dix jours, ils en ressortent avec la conviction qu'on peut s'amu-ser en français, etc. et avec la détermination de faire quelque chose ${ }^{85}$.

Le père jésuite Albert Regimbal, depuis plusieurs années déjà, avait systématiquement barré la route à l'AJFo dans ses tentatives

84. «Procès-verbal de la réunion spéciale du Bureau central des 4 et 5 juillet 1964 », CRCCF, FAJFO, C9/11/8. Le SyNOJ organisa de nombreuses autres activités sociales et culturelles, dont une série de causeries diffusées sur les ondes de la station de radio hulloise $\mathrm{CKCH}$.

85. Lettre d'Onil M. Perrier, s.m.m., vicaire de la paroisse Saint-Vincent-dePaul de North-Bay, à Rémy Beauregard, 4 septembre 1964, CRCCF, FAJFO, C9/8/2. 
d'étendre son influence dans le Moyen-Nord en général et à Sudbury en particulier. L'orientation de son Centre des jeunes s'inspirait d'abord et avant tout de la doctrine sociale de l'Église et des préceptes de l'Action catholique et avait tendance à réduire la question nationale à la portion congrue. En effet, Regimbal semble avoir été assez largement hostile à l'idéologie que véhiculait l'élite nationaliste d'Ottawa et avait plutôt choisi d'axer son programme sur l'organisation d'activités récréatives et sportives pour la jeunesse franco-ontarienne de sa région ${ }^{86}$. Le père Perrier, pour sa part, tout en appuyant les efforts de l'AJFO, semblait nettement préférer les méthodes du père Regimbal et exhortait l'Association à s'en inspirer afin de rejoindre un nombre plus élevé de jeunes. Au lendemain du ralliement de North-Bay, il ne dissimula pas, non plus, sa déception vis-à-vis de la tournure qu'avait prise l'événement. Alors qu'il s'était attendu à une « rencontre fraternelle où il [devait] y [avoir] peu d'étude mais une intense vie de groupe », il avait constaté, à son grand dam, que « l'accent [avait été] mis sur les études ». L'atmosphère aurait été « pesante », Perrier subodorant un certain « snobisme » de la part des représentants du Bureau central et déplorant que l'AJfo n'eût pas réussi à expliquer aux jeunes la pertinence de sa mission ni la nécessité, pour eux, de se joindre au mouvement. Plutôt que de se mettre à leur écoute, les membres du Bureau central avaient cherché à leur imposer un programme et une philosophie qui leur étaient largement étrangers. Voilà qui était une façon bien peu efficace, conclut-il, d'exercer son leadership : « Quand la question d'autorité est posée, leur reprocha-t-il, qu'il soit clair qu'il s'agit d'une autorité démocratique et amicale, comme l'Église essaie de s'en donner à l'heure actuelle... respectueuse des groupes et des personnes, qui loin de vouloir imposer ses vues et ses décisions, invite chacun à s'exprimer, à partager idées et initiatives et

86. La question des rapports entre l'AJFo et les différentes régions de l'Ontario français, dont le Nord, déborde le cadre de cet article. Nous en avons traité, cependant, dans « Une guerre sourde : la rivalité Ottawa - Sudbury et la jeunesse franco-ontarienne (1949-1965) », Québec Studies, no 46, automne 2008 - hiver 2009, p. 19-31. 
à travailler ensemble ${ }^{87}$. \ Autrement dit, l'AJFo devait s'inspirer de l'Église de Vatican II et faire sien son mot d'ordre : aggiornamento. L'élitisme, le traditionalisme et le nationalisme que Perrier croyait toujours percevoir à l'AJFO semblaient bel et bien s'inscrire en faux contre le zeitgeist des années 1960.

Tous n'étaient pas de cet avis, cependant. En effet, pour d'autres, l'AJfo avait déjà poussé beaucoup trop loin sa logique " démocratisante » en cherchant à se transformer en mouvement de masse voué essentiellement à l'organisation de loisirs et d'activités récréatives, au détriment de l'étude nationale et de la formation d'une élite dirigeante cultivée et éclairée. L'ancien secrétaire général de l'Association, Michel Gaulin, était de ceuxlà. Vers la fin de 1964, le Bureau central voulut organiser de grandes festivités pour célébrer le quinzième anniversaire de 1'Association et invita plusieurs de ses anciens membres à y prendre part, dont Gaulin. Depuis Harvard, où il avait entamé des études doctorales en lettres françaises, ce dernier opposa un refus catégorique à l'invitation du Bureau central dans une lettre massacrante qui lui permit de dénoncer vertement l'orientation idéologique récente de l'AJFo :

Panem et circenses - du pain et des jeux. Voilà ce qu'on trouve de mieux à donner, ces années-ci, à une jeunesse francoontarienne qui s'enlise de plus en plus dans les ténèbres de l'ignorance et dans la fange de l'inconscience. C'est donc en amusant la jeunesse - même si on le fait en français (ou en joual !) qu'on espère résoudre la crise aiguë que traverse présentement le Canada. Belle idée ! C'est en lui enseignant, à toute fin pratique [sic], le mépris des valeurs de l'esprit et de la connaissance de soi par la culture qu'on espère donner à cette jeunesse la force de représenter demain la nation canadiennefrançaise sur la place publique. Belle idée encore, et qui annonce de merveilleux résultats ! C'est triste à faire pleurer $^{88}$ !

87. Lettre d'Onil M. Perrier, s.m.m., vicaire de la paroisse Saint-Vincent-dePaul de North-Bay, à Rémy Beauregard, 26 octobre 1964, CRCCF, FAJFO, C9/8/2.

88. Lettre de Michel Gaulin aux membres du Bureau central, 8 décembre 1964, CRCCF, FAJFO, C9/18/4. 
À la différence des Canadiens français, poursuivit Gaulin, les Canadiens anglais, qui fréquentaient les plus grandes universités, avaient compris, eux, l'importance de la culture et de l'érudition. Le danger était grand, ironisa-t-il, qu'ils en eussent assez de « notre crétinisme » et qu'ils « nous envo[yass]ent paître en nous disant : "Eh bien dansez maintenant" ${ }^{\prime 9} »$. L'Association, d'après lui, était « agonisante », elle avait renié à la fois son passé et sa mission. Devant l'obstacle, elle avait choisi la fuite :

L'Association de la jeunesse franco-ontarienne a été appelée, un jour, à une grande mission. Devant la difficulté de cette mission, elle a choisi de tourner le dos et d'amuser ses adhérents. Plutôt que d'élever la masse vers les sommets de l'idéal, elle a choisi de ramper et de rassasier la plèbe. Qu'elle repose donc en paix, et que son fantôme cesse à jamais de hanter les arcanes de l'Ontario français! Elle fait présentement plus de tort que de bien à la collectivité ${ }^{90}$.

Lors de la réunion suivante du Bureau central, les membres se contentèrent de prendre note des admonestations de leur ancien secrétaire général, sans plus ${ }^{91}$. Dans d'autres circonstances, ils auraient peut-être réagi avec plus de vigueur et d'indignation, mais en ce 16 décembre 1964, ils avaient l'esprit ailleurs. Le but de cette rencontre était de discuter d'un mémoire que leur avait soumis le président général, Carol Kelly, qui avait menacé de démissionner dans l'éventualité où le Bureau central en aurait refusé les recommandations. Le cœur du problème? La nature des rapports entre l'Ajfo et le Synoj et, plus largement, l'orientation générale du mouvement. Tout en soulignant la nécessité d'un organisme comme le Syndicat national des œuvres de la jeunesse, Kelly estimait que l'AJfo et le Synos étaient devenus trop intimement liés l'un à l'autre, situation qui avait engendré beaucoup de « confusion », surtout en ce qui avait trait au mandat de la première. L'AJFo devait prendre les grands

89. Ibid.

90. Ibid.

91. «Procès-verbal de la réunion spéciale du Bureau central du 16 décembre 1964 tenue dans la salle de réunion de la Maison franco-ontarienne », CRCCF, FAJFO, C9/11/8. 
moyens pour retrouver sa vigueur et son dynamisme d'antan et revenir à sa mission initiale, celle de « préparer une relève » en collaboration avec ses "aînés », dont elle avait besoin des « conseils » : « Pour préparer cette relève, il faut la grouper, la faire travailler et la rendre consciente de la position des [F]ranco$[\mathrm{O}]$ ntariens $^{92}$. » Kelly proposa, entre autres choses, que l'AJFO rompît tous ses liens avec le SyNOJ, que les membres du Bureau central redevinssent, comme autrefois, des « pionniers » et qu'ils se rapprochassent de l'AcFÉo. Kelly proposait, autrement dit, que l'AJFo fît un retour aux sources, qu'elle oubliât son engouement récent pour les loisirs et qu'elle revînt à la formule qui, dans les années 1950, avait fait son succès.

L'Association était en crise. Kelly exigea que les membres du Bureau central qui faisaient aussi partie du comité directeur du Syndicat choisissent leur camp : ou bien ils se consacreraient corps et âme à l'AJFO, ou bien ils passeraient définitivement du côté du Synoj. Gilles Blache, le fondateur du Bistro, refusa de s'en laisser imposer ainsi : si l'AJFo se trouvait au bord du gouffre, rétorqua-t-il, il ne fallait pas en imputer la faute au SyNOJ. Les membres les plus actifs et les plus dynamiques du Bureau central étaient, justement, ceux-là mêmes qui participaient également aux activités du Syndicat. L'AJFo avait donc mieux à faire que de chercher des coupables parmi ses membres les plus dévoués : il fallait plutôt qu'elle effectuât son propre examen de conscience, sans chercher de bouc émissaire pour son échec ${ }^{93}$. Ce débat houleux, au final, entraîna la démission de trois membres du Bureau central : Gilles Blache, Jean-Claude Carisse et Jean-Louis Renaud. La suite de cette histoire est cependant difficile à reconstruire, faute de sources. Le Bureau central, qui semble s'être

92. Carol Kelly, « Mémoire présenté au Bureau central de l'Association de la jeunesse franco-ontarienne lors de sa réunion tenue samedi le 19 [sic] décembre à la salle de réunion de la Maison franco-ontarienne », annexé au «Procès-verbal de la réunion spéciale du Bureau central du 16 décembre 1964 tenue dans la salle de réunion de la Maison franco-ontarienne », CRCCF, FAJFO, C9/11/8.

93. «Procès-verbal de la réunion spéciale du Bureau central du 16 décembre 1964 tenue dans la salle de réunion de la Maison franco-ontarienne », CRCCF, FAJFO, C9/11/8. 
carrément écroulé au lendemain de cette rencontre fatidique, ne se réunirait plus avant le 14 avril 1965, soit après quatre mois d'inactivité. Entre-temps, Carol Kelly aurait cédé la présidence générale à Denis Bélanger et accepté le poste de trésorier - qu'il n'occuperait cependant que brièvement -, tandis que Jean-Louis Renaud, l'un des trois membres démissionnaires, aurait retrouvé sa place au Bureau central ${ }^{94}$.

La crise s'était résorbée, momentanément, du moins. L'affrontement entre réformateurs et traditionalistes semblait s'être soldé par la victoire au moins partielle des premiers sur les seconds : l'AJfo avait choisi d'appuyer le SYNOJ. Le Bureau central réussit même à obtenir une subvention de $3000 \$$ du Service du Canada français d'outre-frontières ( $\mathrm{SCFOF}$ ) du ministère des Affaires culturelles du Québec pour la réorganisation du Syndicat, dont le but serait dorénavant de « s'affilier ou [de] fonder des organismes qui, chacun dans [sa] sphère propre, offrir[ai]ent les services requis pour la jeunesse francophone ${ }^{95} \gg$. Dans le bilan qu'il dressa du projet au SCFOF, en janvier 1966, le Bureau central rapporta que la subvention avait servi à mettre sur pied deux autres « Bistros » à Ottawa et à préparer le terrain à la fondation de boîtes de nuit semblables à Cornwall, à Hawkesbury et à Welland. On avoua, dans le même souffle, que l'AJfo avait connu « une crise aiguë » au cours des mois précédents. Elle « venait de répondre aux questions idéologiques qu'elle [s'était posées] depuis quelque temps », alors qu'elle avait eu beaucoup de mal à faire accepter

94. «Procès-verbal de la rencontre du 14 avril 1965 [du Bureau central de l'AJFO] », CRCCF, FAJFO, C9/12/1. Jean-Claude Carisse reviendrait également à l'AJFO à titre de secrétaire général.

95. Lettres de Guy Frégault, sous-ministre des Affaires culturelles du Québec, à Jules Labelle, 21 avril 1965; de Jean-Louis Renaud à Guy Frégault, 28 avril 1965, CRCCF, FAJFO, C9/18/5. Il est intéressant de noter qu'en marge de la lettre de Frégault à Labelle, Gilles Blache transcrivit la note manuscrite suivante : «Heureux que nos démarches aideront l'AJFo dans sa nouvelle orientation. - Bon succès et [b]onne chance. » Le fondateur du Bistro était-il passé à l'emploi du ministère des Affaires culturelles du Québec et avait-il aidé l'AJFo à obtenir cette subvention pour la réorganisation du SYNOJ ? À la réunion suivante du Bureau central, on exprima quelques réserves envers l'attitude parfois dirigiste de cet ancien membre de l'AJFo (« Procèsverbal de la réunion du 17 mai 1965 [du Bureau central de l'AJFo] », CRCCF, FAJFO, C9/12/1). 
sa formule auprès des jeunes : le nombre de cercles affiliés, en effet, avait fondu de moitié, depuis l'année précédente, pour se situer à cinq, dont trois seulement qui fonctionnaient avec une certaine régularité. L'Association « était presque disparue de la scène franco-ontarienne », avoua-t-on candidement. Dans un tel contexte, l'AJFO devait à tout prix accorder la priorité à ses efforts de restructuration et d'expansion ${ }^{96}$. Elle était encore loin de se douter, en ce début de 1966, qu'elle s'apprêtait à semer elle-même le germe de sa propre perte.

\section{II - De l' Ajfo À Direction-Jeunesse (1966-1971)}

\section{A. La Conférence-consultation de la jeunesse franco-ontarienne (CCJFO)}

L'AJFO avait-elle complètement écarté la solution du « retour aux sources » qu'avait proposée, en décembre 1964, Carol Kelly? Son appui au Synos et à l'initiative des Bistros signifiait-il qu'elle avait consommé sa rupture avec sa mission initiale, celle de former une élite dirigeante par le biais de l'étude nationale et religieuse ? Rien n'était moins clair. Encore une fois, il semble que les dirigeants de l'Association aient voulu ménager la chèvre et le chou : incapables, de toute évidence, d'abandonner complètement l'idée de former la future élite dirigeante de l'Ontario français, ils continuaient de valoriser, en même temps, la méthode des loisirs et des activités récréatives pour attirer vers l'Association la masse des jeunes Franco-Ontariens. La décision prise par l'AJFO de soutenir le SyNOJ n'avait cependant pas éliminé le problème de l'enchevêtrement des deux organismes, dont il fallait démêler les relations. À l'été 1965, le vice-président à l'administration, Jules Labelle, proposa au Bureau central un projet pour le moins ambitieux consistant à transformer le SYNOJ en véritable «fédération » pour regrouper carrément l'ensemble des

96. «Rapport moral sur l'utilisation de la subvention de $3000,00 \$$ accordée par le Ministère des Affaires culturelles du Québec à l'AJFO en vue de l'aider à organiser le Syndicat d'Euvres de jeunesse », 29 janvier 1966, CRCCF, FAJFO, C9/12/3. 
organismes et des associations de jeunes de l'Ontario français, y compris 1'AJFO ${ }^{97}$. Le nouveau SYNOJ, selon le projet de Labelle, serait devenu l'organisme-parapluie regroupant le Bureau central de l'AJFo et les représentants des autres organismes de jeunesse. L'AJFo aurait continué de regrouper des cellules non plus locales ou paroissiales, mais désormais régionales et se serait consacrée exclusivement, dans sa programmation, à l'étude. Labelle proposait, somme toute, que l'AJFO devînt une sorte de pépinière de « chefs » pour les autres organismes de jeunesse rattachés à la fédération.

La réflexion autour de ce projet avait débuté dès le printemps précédent, au moment même où 1'AJfo avait commencé à reprendre du poil de la bête. Au lendemain de la crise qui l'avait paralysée pendant quatre mois, le Bureau central avait effectué une demande de financement auprès de Pierre Gravelle, « administrateur » de l'Association culturelle d'Ontario (Aco) afin d'obtenir un coup de pouce dans le processus de restructuration ${ }^{98}$. L'Aco était, selon toute vraisemblance, le prête-nom de l'Ordre franco-ontarien, qui avait succédé à l'Ordre de Jacques-Cartier après la dissolution de ce dernier en février de la même année ${ }^{99}$. Gravelle avait été, par ailleurs, un membre actif de l'AJFo quelques années plus tôt et avait même précédé Michel Gaulin au secrétariat général. Il répondit, toutefois, qu'il était impossible que l'ACO acquiesçât à la demande du Bureau central en raison des efforts de réorganisation qu'elle avait elle-même entrepris ces derniers temps (« pour des raisons que vous connaissez déjà », précisat-il). Il conseilla cependant à Jules Labelle de faire preuve de prudence dans son projet de fédération, tout en l'encourageant à

97. «Projet d'une fédération des organismes et des associations de jeunes de l'Ontario français », annexé au « Procès-verbal de la réunion du 29 juillet 1965 [du Bureau central de l'AJfo] », CRCCF, FAJFO, C9/12/1.

98. Lettre de Pierre Gravelle, administrateur [de l'Association culturelle d'Ontario], à Jean-Louis Renaud, secrétaire général de l'AJfo, 4 juin 1965, CRCCF, FAJFO, C9/18/5.

99. Les limites de cette étude ne nous permettent pas d'aborder les liens entre l'AJFo, l'Ordre de Jacques-Cartier et les autres membres du réseau associatif francoontarien. Cette question fera cependant l'objet d'une analyse ultérieure. 
organiser une « consultation préliminaire auprès des organismes susceptibles de s'affilier à la Fédération ${ }^{100} »$.

La chose se ferait, mais pas avant que deux années ne se fussent écoulées. L'idée d'une vaste consultation, baptisée « Opération - Jeunesse '67 », fut lancée au congrès d'octobre 1965 afin de faire enquête sur les « problèmes spécifiques » de la jeunesse franco-ontarienne » et d'étudier les différentes sources de financement possibles pour la réalisation de ses projets ${ }^{101}$. La proposition, préparée par Jules Labelle, prévoyait qu'on regroupât l'AJfo, les étudiants de l'Université d'Ottawa, les élèves de l'école secondaire de l'université et de l'Académie de La Salle, ainsi que les mouvements d'Action catholique ${ }^{102}$. Les quelque 90 délégués qui participèrent au congrès de 1965 décidèrent, toutefois, que l'initiative méritait qu'on l'étudiât davantage avant qu'on ne pût espérer la réaliser. Ironiquement, c'est de Sudbury, là où l'AJfO avait eu tant de mal à s'implanter, que viendrait, quoique indirectement, le coup de pouce devant la conduire à exécuter le projet. En juin 1966, le Centre des jeunes de Sudbury, de concert avec la Commission du Centenaire et l'Association canadienne des centres de loisirs (ACCL, organisme dont l'AJFo fut membre, à quelques reprises, durant les années 1960), lança une vaste « conférence-consultation de groupements de jeunesse de langue française des provinces minoritaires ${ }^{103}$ » à laquelle 1'AJfo fut conviée. L'événement eut lieu à Sudbury en juin 1966 et se répéterait à Maillardville (Colombie-Britannique) en décembre de la même année et à Pointe-de-l'Église (Nouvelle-Écosse) en septembre 1967, toujours sous l'œil vigilant du père Regimbal. Si le surmenage empêcha l'AJFo d'assurer sa présence à la conférence de Sudbury, elle ne rata cependant pas l'occasion de

100. Lettre de Pierre Gravelle, administrateur [de l'Association culturelle d'Ontario], à Jules Labelle, vice-président à l'administration de l'AJFO, 5 août 1965, CRCCF, FAJFO, C9/18/5.

101. « Opération - Jeunesse ' 67 », document annexé au « Rapport [du] $10^{e}$ congrès général [de l'AJfo, tenu] les 9, 10 et 11 octobre 1965 », CRCCF, FAJFO, C9/8/6.

102. Auxquels devaient s'ajouter quelques représentants indépendants et adultes.

103. « Procès-verbal de la réunion du comité de régie [du Bureau central de l'AJFo] tenue le $1^{\text {er }}$ juin 1966 », CRCCF, FAJFO, C9/12/3. 
participer aux rencontres subséquentes ${ }^{104}$. Et c'est en marge de ces rassemblements qu'elle organiserait, en 1967, un événement qui représenterait, bien malgré elle, le premier pas sur le chemin de son déclin irrémédiable, soit la «Conférence-consultation de la jeunesse franco-ontarienne » $(\mathrm{C} \mathrm{CJFO})$.

Lors de son congrès d'avril 1966, l'ACFÉo invita l'AJFo à effectuer un inventaire de l'ensemble des mouvements de jeunesse franco-ontariens, projet qui découlait de la tentative avortée, l'année précédente, de transformer le SyNOJ en fédération provinciale $^{105}$. L'Association d'éducation n'était pas demeurée indifférente devant les difficultés qu'éprouvait, depuis quelque temps, son « rejeton » et avait même mis sur pied, dès janvier 1964, un «comité adulte-jeunesse » pour tenter d'y voir plus clair ${ }^{106}$. Profitant de l'engouement suscité par les conférencesconsultation de la jeunesse francophone hors Québec qu'organisait le Centre des jeunes de Sudbury au même moment (et souhaitant peut-être, bien que cela ne soit pas facile à démontrer, éviter de se faire damer le pion par le père Regimbal), l'AcFÉO envisageait probablement le projet d'inventaire comme une façon de contribuer à « redynamiser » une AJfo devenue chancelante en lui confiant une mission d'envergure et rassembleuse. Le Bureau central semblait du même avis et accepta sans hésitation l'invitation de son "aînée " ${ }^{107}$. L'enquête se fit en deux temps. L'Association se renseigna d'abord sur le nombre d'organismes de jeunesse « dans toutes les localités et/ou paroisses ayant une

104. Que la collaboration ait été plus grande entre l'AJFo et le Centre des jeunes après plusieurs années de méfiance et de relations difficiles ne peut-il pas être considéré comme un indice de ce que l'Association avait pris certaines distances, encore une fois, vis-à-vis du nationalisme canadien-français que semblait abhorrer le père Regimbal?

105. «Inventaire sommaire des associations de jeunesse en Ontario », 6 mars 1967, CrCCF, FAJFo, C9/19/3.

106. «Rapport bi[s]annuel des activités de l'AJFO », présenté lors du Dixième Congrès général tenu à Eastview (Vanier) en octobre 1965, CRCCF, FAJFO, C9/8/6. Ce comité était dirigé par Jacques Leduc, vice-président de l'AcFÉo et conseiller laïc du Bureau central de l'AJfo.

107. « Procès-verbal de l'assemblée générale du 20 juillet 1966 [du Bureau central de l'AJfo] », CRCCF, FAJfo, C9/12/4 ; « Procès-verbal de l'assemblée du Bureau central [de l'AJfo] du 15 décembre 1966, CrCCF, FAJFO, C9/12/4. 
population française » en prenant appui sur les ressources du réseau paroissial franco-ontarien et des sections locales de l'ACFÉO ${ }^{108}$. Cette démarche lui permit de découvrir l'existence d'au moins 68 organismes de jeunesse œuvrant en Ontario français dans divers domaines ${ }^{109}$. Armée de ces résultats préliminaires, l'AJfo obtint de l'ACFÉo une somme de 4000 \$ qui lui permit d'organiser une grande rencontre de ces associations à NorthBay en mai $1967^{110}$. La deuxième étape de l'enquête consistait donc à intervenir directement auprès des associations recensées afin de les encourager à participer au rassemblement de NorthBay. L'AJFo organisa quatre rencontres préliminaires (à London, à Sudbury, à Cochrane et à Ottawa) et fit parvenir aux divers organismes un questionnaire les interrogeant sur leur histoire, leurs réalisations, leurs projets, leur connaissance du milieu franco-ontarien et, finalement, leur opinion face à la tenue de la Conférence-consultation en mai ${ }^{111}$. Sur les 62 répondants, 55 $(88,7 \%)$ se dirent favorables à la tenue de la rencontre, $54(87 \%)$ prévoyaient y participer, alors que $45(72,6 \%)$ jugèrent opportune l'idée de former une fédération des mouvements de jeunesse franco-ontariens ${ }^{112}$.

108. « Inventaire sommaire des associations de jeunesse en Ontario », 6 mars 1967, CrCCF, FaJfo, C9/19/3 ; Franc-Jeu, mars 1967, p. 8, CRCCF, FAJfo, C9/36/7.

109. Soixante-huit groupes répartis de la manière suivante : 29 groupes de loisirs, 18 associations d'Action catholique, 10 organismes étudiants, 7 cercles de l'AJFo et 4 autres regroupements non identifiés.

110. « Procès-verbal de l'assemblée régulière du Bureau central [de l'AJfo], le 6 mars 1967 », CRCCF, FAJfo, C9/12/5; communiqué de presse sur la tenue de la Conférence-consultation de la jeunesse franco-ontarienne, 26 avril 1967, CRCCF, FAJFO, C9/9/1. C'est Conrad Lavigne, de Timmins, qui fut chargé, par l'AcFÉo, de diriger la « campagne-éclair » organisée pour prélever cette somme au profit de l'AJFo (« Procèsverbal de la réunion du comité exécutif [de l'ACFÉo] tenue à la Maison franco-ontarienne, le 15 mars 1967, à 7 heures 30 du soir », CRCCF, Fonds Rémy-Beauregard (dorénavant FRB), P45/11/7). Soulignons que la rencontre de Sudbury eut lieu dans l'édifice du Centre des jeunes de Sudbury, ce qui semble indiquer, encore une fois, que l'ancienne rivalité entre l'organisme de Regimbal et l'AJfo s'était considérablement estompée.

111. "Conférence-consultation de la jeunesse franco-ontarienne; re : rencontres préliminaires du samedi 6 mai 1967 », CRCCF, FRB, P45/11/7 ; « Rapport d'enquête sur les mouvements de jeunesse franco-ontariens », 15 mai 1967, CRCCF, FAJFO, C9/9/1.

112. « Rapport d'enquête sur les mouvements de jeunesse franco-ontariens », 15 mai 1967, CRCCF, FAJFO, C9/9/1. 
La Conférence-consultation de la jeunesse franco-ontarienne eut bel et bien lieu à North-Bay du 20 au 22 mai 1967. Le comité organisateur, que présidait Rémy Beauregard ${ }^{113}$, avait expédié plus de 200 invitations à des représentants de clubs de loisirs, de mouvements d'Action catholique, d'associations étudiantes, d'écoles secondaires, etc. Il en vint, au total, plus d'une centaine, résultat que les organisateurs considérèrent comme satisfaisant, d'autant plus que les délégués provenaient des quatre coins de l'Ontario français, sans exception ${ }^{114}$. Dans l'ensemble, les débats portèrent sur la viabilité et la nécessité de la création d'une fédération provinciale des mouvements de jeunesse. Il est clair que les organisateurs souhaitaient susciter un sentiment d'appartenance à « une communauté de jeunesse francoontarienne ». Ce sentiment, en effet, ne semblait pas faire l'unanimité, plusieurs des participants déplorant l'absence de structures qui en eussent favorisé l'émergence en unifiant la masse des jeunes Franco-Ontariens. Le consensus était plus grand, cependant, sur l'existence d'une « communauté de fait », fondée sur le partage d'une même langue et d'une même culture (la foi ayant été complètement évacuée, manifestement, de cette conception de l'identité franco-ontarienne). Le défi consistait donc à briser l'isolement des jeunes Franco-Ontariens et à favoriser la communication par l'établissement d'une structure fédérative, projet qui fut tout de suite mis à l'étude. On forma donc un «Comité provisoire » qui veillerait à préparer le terrain pour la fondation d'une éventuelle fédération provinciale. Ce comité serait composé de huit membres, dont quatre délégués des grandes régions de l'Ontario français (Est, Sud, Grand Nord, Petit Nord) et encore quatre représentants des divers champs d'activité dans lesquels œuvraient les mouvements de jeunesse (études, loisirs, culture, action sociale). Le poste de président du Comité provisoire

113. À ce moment, Beauregard était membre à la fois du Bureau central de l'AJFo et secrétaire adjoint de l'AcFÉo.

114. « Procès-verbal de la Conférence-consultation de la jeunesse francoontarienne tenue à l'école St-Thomas, rue King, North[-]Bay, Ontario, les 20, 21 et 22 mai 1967 », CRCCF, FAJFO, C9/9/2. 
fut confié à Rémy Beauregard. Les délégués exprimèrent clairement la volonté que la démarche du Comité provisoire fût transparente, démocratique, non autoritaire et qu'elle respectât les particularités régionales.

Dès sa formation, le Comité provisoire - ou tout simplement la CCJFo, comme on l'appela le plus souvent - se considérait comme la seule voix autorisée à parler au nom de la jeunesse franco-ontarienne. Le mandat qu'on lui avait confié avait été, tout simplement, de jeter les bases d'une fédération provinciale des mouvements de jeunesse, mais qu'à cela ne tienne : la CCJFO multiplia les interventions dans divers dossiers en adoptant un ton qui contrastait singulièrement, au demeurant, avec celui de l'AJfo. Peu de temps après le rassemblement de mai 1967, la CCJfo se pencha, par exemple, sur le rapport du comité de l'Assemblée législative de l'Ontario sur la jeunesse que présidait, depuis 1964, le député conservateur Charles Apps ${ }^{115}$. C'est ce rapport qui conduirait le gouvernement Robarts à créer un Service des Loisirs et de la Jeunesse au ministère de l'Éducation. La CCJFO produisit une étude qui, en huit pages, constituait une véritable dénonciation en règle de l'attitude « paternaliste » du comité Apps vis-à-vis de la jeunesse. Elle accusa le comité d'avoir refusé d'envisager la situation de la masse des jeunes Ontariens, qui était destinée à composer à très brève échéance plus de la moitié de la population totale de la province, pour se concentrer à peu près uniquement sur les problèmes de la jeunesse « anormale » ou « délinquante », c'est-à-dire celle qui souffrait de « tares physiques ou intellectuelles ${ }^{116} »$. Dans un même ordre d'idées, la CCJFo déplora que le comité Apps eût complètement passé sous silence les problèmes de la classe ouvrière, dont était issue, encore une fois, la masse des jeunes Ontariens. On reconnaît facilement, à la lecture de cette étude, l'influence croissante qu'exerçaient quelques-uns des grands thèmes de la contre-culture et de

115. Report of the Ontario Legislature's Select Committee on Youth, Kingston, Hanson \& Edgar, 1967, 408 p.

116. Alain Duhamel, «Étude [de la C CJFo] sur le rapport du comité d'enquête de la [C]hambre législative [de l'Ontario] sur la jeunesse », [1967], CRCCF, FAJFO, C9/9/2. 
l'idéologie de participation sur l'esprit des dirigeants de la CCJFO : critique de l'establishment, conscience d'appartenir à une génération nombreuse et prête à ébranler le traditionalisme des sociétés occidentales, valorisation de la réalité vécue des masses populaires et ouvrières selon une perspective « marxisante », etc. Les jeunes étaient encore présentés comme des adultes en devenir, mais leur identité n'était plus réduite à cette seule dimension. $\mathrm{Au}$ contraire, les enfants du baby boom, en Ontario français comme ailleurs, représentaient une catégorie sociale autonome se croyant autorisée à investir la place publique et à faire valoir ses intérêts comme ses prédécesseurs ne l'avaient encore jamais fait. La société n'avait d'autre choix, semblait-on dire, que de composer avec cette nouvelle réalité.

La CCJfo déplora tout aussi vertement le fait que le comité Apps eût complètement ignoré l'existence d'une jeunesse spécifiquement franco-ontarienne dans la province, bien qu'il eût formulé des recommandations visant les « Néo-[C]anadiens, les [I]ndiens et les [E] squimaux ${ }^{117} \gg$. Il fallait que l'éventuel ministère de la Jeunesse eût la responsabilité de freiner l'assimilation de la jeunesse franco-ontarienne mais, en revanche, le gouvernement ontarien ne devait « en aucun temps et sous aucune considération [sic] s'occuper de la culture française ${ }^{118} \gg$ directement. La CCJFO exigea plutôt, sur un ton fort revendicateur, que l'État finançât « inconditionnellement » le développement de la culture francoontarienne, mais sans plus : les jeunes Franco-Ontariens ne se reconnaissaient d'autre maître qu'eux-mêmes et les autorités politiques ou administratives n'avaient pas à se mêler de l'usage qu'ils feraient des subventions ministérielles obtenues. Dans le même souffle, cependant, la C CJFO reconnut que, si le regard des membres du comité Apps ne s'était pas posé spécifiquement sur la jeunesse franco-ontarienne, la faute en était au moins partiellement imputable aux Franco-Ontariens eux-mêmes : sur les quelque 700 mémoires que reçut le comité, dix seulement

117. Ibid., p. 3.

118. Ibid., p. 7. 
provenaient d'associations françaises. La CCJFO profita de ce constat pour décocher une flèche pour le moins inattendue en direction de sa génitrice, l'AJFo : durant les travaux du comité, cette dernière avait « brill[é] par son absence ${ }^{119}$ » et donc manqué à son devoir de représentation, fallait-il comprendre. La nécessité d'une nouvelle association provinciale pour regrouper l'ensemble de la jeunesse franco-ontarienne paraissait plus grande que jamais, aux yeux de la CCJFO. Pareil organisme était destiné, selon elle, à devenir « un agent revendicateur du groupe franco-ontarien ${ }^{120}$ » et un intermédiaire efficace entre le gouvernement provincial et la jeunesse de l'Ontario français. L'AJFO, de toute évidence, était incapable de s'acquitter de cette tâche de représentation et d'organisation auprès des instances politiques de la province.

La table était mise pour un conflit entre l'AJFo et son rejeton. Les deux organismes, pourtant, demeuraient encore étroitement liés. Des $4000 \$$ obtenus de l'ACFÉO pour l'organisation du rassemblement de mai 1967, 1'AJFo en transféra, après coup, les trois quarts à la $\mathrm{C}$ CJFO, les frais de transport des délégués ne s'étant élevés qu'à 1000 \$. L'Association avait également investi 1400 \$ de ses propres fonds dans l'organisation de la rencontre et mis son bureau et son équipement à la disposition de la CCJFo, « moyennant un certain remboursement ${ }^{121} »$. Le président du Comité provisoire, Rémy Beauregard, continuait, par ailleurs, de siéger au Bureau central de l'AJfo, bien qu'il eût abandonné son poste de directeur de l'expansion afin de se consacrer entièrement à ses nouvelles fonctions ${ }^{122}$. Il n'est pas impossible, cependant, que l'AJFo ait craint que la CCJFo ne la devançât dans le domaine de la représentation politique. Dès juin 1967, soit un mois après

119. Ibid., p. 3.

120. Ibid., p. 8 .

121. En février 1968, l'AJFo permit également à la CCJFo d'organiser son tirage annuel (qui constituait l'une de ses principales sources de revenus) et de conserver $60 \%$ des profits réalisés («Procès-verbal de la réunion du Bureau central de l'Association de la Jeunesse Franco-Ontarienne [sic] tenue lundi le 12 février 1968 à la Maison Franco-Ontarienne [sic] », CRCCF, FAJFO, C9/12/6).

122. Pour tous ces renseignements, voir : "Procès-verbal, assemblée régulière du Bureau central [de l'AJfo], le 29 juin 1967 », CRCCF, FAJfo, C9/12/5. 
la tenue de la Conférence-consultation, le Bureau central discuta de la possibilité de faire de la question des écoles secondaires publiques françaises, dont le gouvernement provincial devait bientôt permettre la création, un « ballon politique ${ }^{123} »$. Le monde franco-ontarien, que l'on présenta comme «croulant», devait « faire quelque chose », alors que l'AJfo, au dire de certains membres du Bureau central, «était dans l'erreur en ne faisant rien ». Il n'est peut-être pas inutile de souligner que ce débat avait été lancé par Pierre Allard, qui avait été secrétaire du comité d'organisation de la Conférence-consultation et qui occupait, depuis, le poste de représentant régional de l'Est au Comité provisoire.

Le Bureau central ne prit aucune décision claire, cependant, par rapport aux écoles secondaires. En réalité, la création du Comité provisoire de la $\mathrm{C}_{\mathrm{CJFO}}$ contribua puissamment à approfondir le dilemme existentiel de l'AJfo. Les choses ne progressaient-elles pas, pourtant, comme le Bureau central l'avait espéré ? N'était-ce pas l'AJfo elle-même qui avait lancé l'idée de fédérer l'ensemble des mouvements de jeunesse francoontariens et le projet n'avançait-il pas bon train ? Sans doute, mais le projet de fédération en vint tout de même à donner naissance, parmi les dirigeants de l'Association, à de nouvelles angoisses par rapport à l'avenir de leur mouvement, aussi incohérent que cela pût paraître. À l'origine, au moment de sa propre fondation, l'AJFo n'avait pas eu l'ambition de regrouper l'ensemble des mouvements de jeunesse franco-ontariens. Il lui avait plutôt incombé de regrouper des individus plutôt que des organismes, et pas n'importe lesquels : elle devait agir surtout auprès des jeunes parmi lesquels l'Ontario français puiserait, plus tard, ses «chefs » nationaux. Il n'avait jamais été question que

123. Ibid. Quelques mois plus tôt, en octobre 1966, l'AJFo avait pris position en faveur de la création d'écoles secondaires françaises publiques, tout en exigeant que des « garanties de confessionnalité » fussent accordées. L'Association devança ainsi l'ACFÉo, qui n'abandonnerait qu'en 1967 l'idée de mettre sur pied un réseau d'écoles secondaires françaises séparées. Voir « Congrès de l'Association de la jeunesse francoontarienne. 8, $9 \& 10$ octobre $1966 »$, CRCCF, FRB, P45/10/2. 
l'AJfo prît la forme d'un mouvement de masse et elle avait dû s'assurer de ne pas empiéter sur le terrain des autres organismes, en particulier celui des mouvements d'Action catholique, auxquels sa propre orientation nationaliste lui interdisait toute participation officielle. Après vingt ans, cependant, le contexte n'était plus le même : l'Action catholique s'éclipserait à peu près entièrement avant la fin des années $1960^{124}$, alors même que l'AjFo, nous l'avons vu, avait déjà pris quelques distances, bien que ce fût de manière ambiguë, par rapport à ses convictions nationalistes et élitistes antérieures. N'aurait-elle pas pu espérer, dès lors, prendre elle-même la forme de ce grand mouvement provincial dont elle avait appelé la création de ses vœux ? Dans l'éventualité où la CCJFo se transformerait en organisme provincial permanent, quel aurait été le champ d'action spécifique de l'AJFo ? De quelle manière se distinguerait-elle du nouvel organisme ? De quelle façon réussirait-elle à maintenir sa pertinence aux yeux de la jeunesse franco-ontarienne ? La montée du mouvement contreculturel parmi les jeunes du monde occidental tout entier à la fin des années 1960 aurait-elle raison, au final, de ce qui restait de sa mission initiale de former la future élite dirigeante de la nation canadienne-française en Ontario ? Quel avenir, somme toute, pour l'AJfo ?

L'organisation de la Conférence-consultation avait peut-être donné un nouveau souffle à l'Association, mais le problème de son orientation générale demeurait entier et continuait de se poser avec une acuité croissante. À l'automne 1967, l'AJfo décida de reporter son congrès afin de ne pas disperser inutilement les énergies et entraver le travail de la CCJFO, qui devait présenter

124. Les mouvements d'Action catholique franco-ontariens attendent toujours leur historien. Cependant, nous savons que la Révolution tranquille signala le déclin, voire la disparition de plusieurs de ces groupes dans le contexte québécois. Rien ne nous permet de supposer que la branche franco-ontarienne de l'Action catholique ait eu la vie plus longue. Voir Louise Bienvenue, Quand la jeunesse entre en scène. L'Action catholique avant la Révolution tranquille, Montréal, Boréal, 2003, 291 p. ; Lucie Piché, Femmes et changement social au Québec. L'Apport de la Jeunesse ouvrière catholique féminine, 1931-1966, Sainte-Foy, Presses de l'Université Laval, 2003, 349 p. ; Gabriel Clément, Histoire de l'Action catholique au Canada français, Montréal, Fides, 1972, $331 \mathrm{p}$. 
son projet de fédération provinciale au début de 1968. L'AJFO reconnut, toutefois, que le ralentissement de ses activités avait des causes encore plus profondes, comme devait l'exprimer candidement son secrétaire général, Jean-Claude Carisse, dans un communiqué destiné aux membres de l'Association :

[C]e n'est pas la moindre des raisons [...] il y a la situation générale. L'AJfo, avouons-le, n'a pas la vigueur et le dynamisme qui la caractérisaient jadis. Depuis quelque temps, le nombre des membres diminue ; le mouvement ne semble plus répondre aux besoins des jeunes.

Le Bureau central - et les cercles, nous en sommes assurés se posent des questions quant à l'avenir du mouvement et la place qu'il doit occuper en Ontario ${ }^{125}$.

Carisse encouragea les membres du mouvement à organiser des « rencontres régionales afin de discuter de l'avenir de l'Association en particulier et [de] la jeunesse franco-ontarienne en général », de concert avec... les organisateurs régionaux de la CCJfo ! Au Bureau central, le vieux débat sur la mission de l'organisme se poursuivait inlassablement. Certains souhaitaient que l'Association se transformât en "mouvement d'étude » regroupant « de petites cellules comportant quelques membres », d'autres préconisaient, au contraire, qu'elle réunît « tous les [F]ranco-[O]ntariens, qu'ils [fussent] médecins, garagistes, avocats, etc., ou encore adultes, adolescents ou enfants ${ }^{126} »$. Jacques de Courville Nicol, un observateur assistant à une réunion extraordinaire du Bureau central en novembre 1967, était de cet avis. Selon lui, la jeunesse avait besoin d'un organisme capable de devenir le « trait d'union entre toutes les régions, sans enlever l'autonomie des associations actuelles ». Mais n'était-ce pas là précisément le mandat de la $\mathrm{C}_{\mathrm{CJFO}}$ ? Tout le monde semblait s'entendre sur une chose, cependant : l'existence et l'utilité de l'AJfo ne paraissaient plus tenir qu'à un fil.

125. Lettre circulaire de Jean-Claude Carisse, secrétaire général de l'AJFo, aux membres de l'Association, 27 août 1967, CRCCF, FAJfo, C9/19/3.

126. « Réunion extraordinaire du Bureau central de l'Association de la Jeunesse Franco-Ontarienne [sic] tenue le 18 novembre 1967 à la Maison Franco-Ontarienne [sic] », CRCCF, FAJFO, C9/12/5. 
La valse-hésitation de l'AJFo quant à son orientation générale semblait destinée à se poursuivre ad nauseam. En décembre 1967, ses dirigeants décidèrent qu'elle se consacrerait désormais entièrement à l'étude, qu'elle abandonnerait toute velléité de se transformer en mouvement de masse et qu'elle ne regrouperait plus, dorénavant, que des jeunes de vingt ans et plus ${ }^{127}$. Cette résolution fut de courte durée, cependant. Lors d'un colloque qu'organisa l'Association à Ottawa en mars 1968 et auquel 30 personnes prirent part, le même affrontement eut lieu entre ceux qui concevaient l'AJfo comme « un mouvement d'élite » devant se limiter « à des noyaux d'individus » et ceux qui auraient voulu en faire « un mouvement de masse très puissant ${ }^{128}$ ». D'autres encore croyaient que c'était plutôt la CCJFO qui était destinée à devenir «LE MOUVEMENT de jeunes en Ontario ${ }^{129}$ ». Il est fort possible que cette opinion ait été émise par l'un des deux représentants de la CCJFO au colloque, Rémy Beauregard et Paul[François] Sylvestre ${ }^{130}$. La rencontre d'Ottawa permit également à l'AJFo de mettre le doigt sur un autre problème devenu fort préoccupant. L'Association ne pouvait plus guère prétendre être un mouvement d'envergure provinciale : bien que ses quartiers généraux fussent situés à Ottawa, la majorité de ses membres provenaient, désormais, du sud de la province. En effet, sur les 30 participants au colloque, pas moins de seize étaient venus de Welland et d'Oshawa. En excluant les sept membres du Bureau central et les deux délégués de la $\mathrm{C}$ CJFO, la rencontre n'avait réussi qu'à susciter l'intérêt de cinq autres participants, tous d'Ottawa ${ }^{131}$.

127. «Procès[-]verbal de la réunion du Bureau central de l'Association de la Jeunesse Franco-Ontarienne [sic] tenue le 9 décembre 1967 », CRCCF, FAJFO, C9/12/5.

128. «Rapport du colloque de l'Association de la jeunesse franco-ontarienne tenu à Ottawa le 23 mars 1963 », CRCCF, FAJFO, C9/9/3.

129. Ibid. Les majuscules sont de l'auteur du rapport.

130. Beauregard, en effet, semblait avoir à peu près entièrement délaissé l'AJFO pour se consacrer exclusivement à la CCJFO.

131. Dans un document qui, vraisemblablement, date de 1968 ou de 1969, on dressa la liste des cercles affiliés à l'AJFo, qui ne se trouvaient plus qu'au nombre de sept, dont trois dans le Sud et quatre dans l'Est. On mentionna également un certain «Centre des jeunes ». Il s'agissait peut-être du centre culturel du père Regimbal, à Sudbury, mais les dirigeants de l'organisme semblaient se situer un peu partout dans la 
Il était clair que l'AjFo n'était plus que l'ombre d'elle-même. Certains de ses membres allèrent jusqu'à proposer que l'Association déménageât son siège social d'Ottawa vers Welland - idée qui ferait, cependant, long feu. Au congrès général de mai 1968, qui eut lieu justement à Welland, les délégués voulurent également amender les statuts de l'organisme afin que ses buts, dorénavant, fussent les suivants : « travailler à la sauvegarde de la culture canadienne-française », « grouper et unir les jeunes [F]ranco-[O]ntariens afin qu'ils puissent se développer librement dans les aspirations conformes à leur origine ethnique » et « voir à développer un programme d'action et de formation dans les loisirs, programme compatible à [sic] notre mentalité de canadienfrançais $[\mathrm{sic}]^{132} \gg$. Les nouveaux statuts devaient être mis à l'essai pendant deux ans afin de déterminer, par la suite, s'il y aurait lieu de relancer le mouvement à l'échelle régionale ou provinciale.

\section{B. L'Association provinciale des mouvements de jeunes de l'Ontario français (APMJOF)}

À l'inverse, la CCJFo avait le vent dans les voiles. À l'automne 1967, elle décida d'entreprendre des démarches pour organiser des « rencontres interprovinciales » afin d'établir des liens avec les jeunes de l'Ouest et des Maritimes, une tâche qui avait longtemps incombé, rappelons-le, à l'AJFO ${ }^{133}$. En ce qui avait trait à son mandat principal, soit la création d'une fédération provinciale, les membres du Comité provisoire de la CCJFo étaient d'avis que «l'essentiel [était] de rejoindre le jeune [F]ranco[O]ntarien afin qu'il [pût] s'exprimer le plus librement possible sur ses problèmes et aspirations ». La méthode de l'« animation de groupe », si prisée à la fin des années 1960 et au début de la

province (Hearst, Pembroke, Toronto, Sudbury, Rockland, Cornwall et Vanier). Voir « Conseil provincial - exécutif [1968 ou 1969] », CRCCF, FAJFO, C9-2/1/7.

132. «Travaux sur la constitution de l'AJFO à être présentés au congrès général convoqué par le Bureau central à Welland pour les 4 et 5 mai 1968 », CrCCF, FAJFo, C9/9/3.

133. « Procès-verbal de l'assemblée du Comité provisoire [de la CCJFo], tenue à la Maison franco-ontarienne, les 14 et 15 octobre 1967 », CRCCF, FrB, P45/11/7. 
décennie suivante, représentait, croyait-on, l'outil le plus efficace et le plus démocratique qui fût pour favoriser le contact souhaité avec la masse des jeunes, qu'il fallait conduire à participer activement à la formation de la fédération provinciale. On tenait également à intervenir non seulement auprès des étudiants, mais aussi - et peut-être surtout - auprès des ouvriers, qui devaient avoir la possibilité de discuter «ouvertement» des loisirs, du monde du travail, de la vie étudiante, etc. Si l'AJfo avait éprouvé beaucoup de mal, depuis le début de la décennie, à choisir entre l'élite et la masse, la $\mathrm{C}_{\mathrm{CJFO}}$, pour sa part, n'avait éprouvé aucune difficulté à trancher la question. Bien qu'elle eût un projet clairement défini auquel elle souhaitait voir adhérer la masse des jeunes, elle percevait son rôle (ostensiblement, du moins) comme consistant à se mettre à leur écoute et à recevoir d'eux ses mots d'ordre. La conception élitiste et hiérarchisée de la société qui avait longtemps été celle de l'AJfo et de l'élite nationaliste traditionaliste du Canada français avait été balayée, de toute évidence, de l'univers mental des dirigeants de la CCJFO, dont le leitmotiv aurait pu être «participation ».

Le Comité provisoire s'engagea, dès lors, à organiser une deuxième Conférence-consultation, toujours à North-Bay, du 18 au 20 mai 1968, environ quinze jours après que l'AJFo eut tenu son propre congrès général à Welland. Il espérait obtenir, cette fois, la participation de 424 délégués et chiffra ses besoins financiers à quelque 16000 \$ qui devaient faire l'objet d'une demande de subvention au Secrétariat d'État ${ }^{134}$. Les gouvernements, manifestement, étaient en passe de devenir des sources de financement incontournables, en cette fin de décennie, sources auxquelles 1'AJfo, historiquement, n'avait guère eu accès. Les organisateurs avaient peut-être vu un peu trop grand, cependant, car le gouvernement fédéral ne leur accorda qu'une subvention de 2500 \$, une somme équivalente, pour le reste, à celle que leur avait fournie le gouvernement provincial. Au troisième rang des bailleurs de fonds de la CCJFO arrivait nulle autre que l'AJFO, avec

134. Ibid. 
ses quelque 1600 \$. L'ACFÉO, pour sa part, se situait loin derrière avec un don de $500 \$ 135$.

Les organisateurs avaient souhaité réunir plus de 400 jeunes au congrès de mai 1968. Il n'en vint qu'une centaine ${ }^{136}$, environ, mais cette participation suffit à jeter les bases de la fédération tant espérée : l'Association provinciale des mouvements de jeunes de l'Ontario français (APMJOF). Pendant trois jours, au moment où la jeunesse de France investissait les rues de Paris et menaçait de faire tomber le régime du général de Gaulle, les délégués à la deuxième $\mathrm{C}_{\mathrm{CJFO}} \mathrm{s}$ 'appliquèrent allègrement à relever les nombreux travers dont la société s'était rendue coupable. En matière d'instruction, on s'en prit au système scolaire, aux cours « trop livresques » qui s'y dispensaient et qui, de l'avis général, rendaient l'étudiant « blasé » en posant un frein au développement de «son sens de la responsabilité et de l'engagement ${ }^{137}$ ». En matière de religion, on constata que la « christianisation fai[sai] $\mathrm{t}$ place de plus en plus à l'humanisation chez les étudiants », tant et si bien que plusieurs d'entre eux avaient carrément rejeté la « pratique religieuse », c'est-à-dire « l'Église [...] ses principes, sa morale, etc. ${ }^{138} »$. Le jeune travailleur, pour sa part, avait besoin d'un « guide » : « Il veut devenir quelqu'un, mais l'adulte d'aujourd'hui ne correspond pas à ce qu'il veut devenir; il se replie sur lui-même. [...] [I]1 n'accepte pas les attitudes paternalistes ${ }^{139}$. » Le travailleur franco-ontarien se serait montré « assez indifférent » vis-à-vis de la politique, qu'il aurait estimée « sale », et du syndicalisme, sans parler du «fait français » qui réussissait rarement à susciter son adhésion : « Il parle français comme il

135. « Liste des donateurs (mai 1967 - mai 1968) [à la CCJFO] », CRCCF, FAJFO, C $9 / 9 / 4$.

136. «Liste des délégués [à la deuxième Conférence-consultation de la jeunesse franco-ontarienne, mai 1968]», CRCCF, FAJFO, C9/9/4.

137. Monique Larouche, «L'Étudiant franco-ontarien », [rapport présenté à la deuxième Conférence-consultation de la jeunesse franco-ontarienne, mai 1968], p. 1, CrCCF, FaJFo, C9/9/4.

138. Ibid., p. 3.

139. Danielle Bourgie, «Le Jeune au travail », [rapport présenté à la deuxième Conférence-consultation de la jeunesse franco-ontarienne, mai 1968], p. 1, CRCCF, FAJFO, C $9 / 9 / 4$. 
est catholique. C'est quelque chose qu'il n'a pas choisi. On travaille en anglais ; l'argent est en anglais. Il est content d'avoir certains droits, mais se battrait-il pour $\operatorname{eux}^{140}$ ?» En réalité, le jeune travailleur franco-ontarien était « le plus délaissé de la société », conclut-on, les loisirs représentant le seul espace de liberté auquel il avait accès.

La problématique des loisirs occupa, d'ailleurs, une place centrale dans les discussions. Si l'AJFo, durant les années 1950, n'avait pas hésité à encourager l'organisation de loisirs « sains ", « formateurs » et en accord avec le caractère « national» des Canadiens français, la réflexion de la CCJFo sur la question se fit à l'extérieur de toute considération morale et nationaliste et se limita à voir dans les loisirs une simple méthode de combat contre l'assimilation linguistique des jeunes Franco-Ontariens : «Les activités sociales composent une grande partie des activités de la jeunesse, qu'elle soit française ou non. La danse est de loin la plus importante de ces activités sociales. Et c'est ici que se situe la plus grande menace au fait français en Ontario à l'heure actuelle ${ }^{141}$. » À l'extérieur des murs de l'école ou du foyer, la jeunesse, libre de toute contrainte, «redev[enait] elle-même », avec comme résultat que « tout se fai[sai]t en anglais ». La solution que l'on proposa : l'animation culturelle. Il eût été possible, de cette manière, de combler les lacunes parfois criantes qui caractérisaient l'offre de «services » culturels dans certaines régions de la province. L'enjeu était crucial : «La culture, expliqua-t-on, fait partie intégrante et inséparable de la nature d'un peuple. Il ne s'agit pas de vivre en français dans un pays à majorité anglaise mais bien de demeurer fidèle à soi-même en conservant et surtout en épanouissant sa culture, en somme, en s'épanouissant soi-même ${ }^{142}$. " La culture et la langue d'une

140. Ibid., p. 4.

141. Jean-Claude Carisse, «Les Loisirs », [rapport présenté à la deuxième Conférence-consultation de la jeunesse franco-ontarienne, mai 1968], CRCCF, FAJFO, C9/9/4.

142. Paul Hébert, « Domaine culturel », [rapport présenté à la deuxième Conférence-consultation de la jeunesse franco-ontarienne, mai 1968], CRCCF, FAJFO, C $9 / 9 / 4$. 
collectivité représentaient donc ses caractéristiques fondamentales. Le nationalisme n'était visiblement pas au rendez-vous, à la deuxième CCJFO, mais l'on s'aperçoit, malgré tout, que l'idée selon laquelle les Franco-Ontariens formaient un «peuple» distinct de la majorité, voire parallèle en Ontario, n'avait pas été entièrement balayée.

Les délégués virent donc dans la fondation d'une fédération provinciale une solution, du moins partielle, à l'ensemble des problèmes que vivait la jeunesse franco-ontarienne. Le mandat du nouvel organisme consisterait à offrir des « services », essentiellement culturels, aux associations qui choisiraient de s'y affilier $^{143}$. Parmi les « services » envisagés, on songea à des " clubs » de livres, de disques ou de cinéma, à des journaux, à l'organisation de tournées d'artistes, etc. Il fallait, par ailleurs, que ces services fussent organisés par un « animateur, social ou culturel ». Ce dernier devait être une « personne de compétence professionnelle » qui « susciterait et conseillerait, dans un groupe donné, une action sociale ou culturelle selon les désirs, aspirations et buts de ce groupe ${ }^{144} \gg$. On prit grand soin, en effet, de souligner le caractère démocratique et représentatif que devait se donner la nouvelle fédération, qui ne devait rien imposer « d'en haut». Il était impératif, au contraire, que cette dernière fît son œuvre «à partir de la base en respectant chaque milieu », compte tenu des disparités régionales parfois très grandes qui menaçaient l'unité des Franco-Ontariens ${ }^{145}$.

Il semble que nous soyons à des années-lumière du contexte intellectuel qui avait prévalu en 1949, au moment de la fondation de l'AJfo. Cette dernière avait reçu le mandat, comme nous l'avons déjà précisé, de donner aux jeunes « la connaissance exacte de ce qu'ils [étaient] comme [F]ranco-[O]ntariens et la volonté d'accorder leur vie aux exigences de cette personnalité franco-

143. « Rapport sur les possibilités d'un organisme provincial », [rapport présenté à la deuxième Conférence-consultation de la jeunesse franco-ontarienne, mai 1968], p. 2, CrccF, FaJfo, C9/9/4.

144. Ibid., p. 2.

145. " Procès-verbal. II ${ }^{\mathrm{e}}$ Conférence-Consultation de la jeunesse francoontarienne, 18, 19 et 20 mai 1968 », p. 4, CRCCF, FAJFO, C9/9/4. 
ontarienne ${ }^{146} »$. Il n'était plus question, de toute évidence, d' « expliquer » aux jeunes « qui » ils étaient. L'esprit « participationniste » des délégués à la deuxième CCJFo et leur souci de représentativité les conduisaient plutôt à se soumettre, du moins en apparence, aux besoins que définirait la jeunesse « à partir de la base ». Pourtant, la démarche de la CCJFo n'était peut-être pas entièrement dépourvue de "dirigisme", pour ainsi dire. La nouvelle fédération provinciale avait, en effet, un projet pour la jeunesse, un projet qui, pour ne pas être aussi clairement défini, du point de vue national et religieux, que celui de l'AJFo vingt ans plus tôt, n'en était pas moins réel : le développement de ce qui manquait le plus, à son avis, à la jeunesse franco-ontarienne, soit une « conscience collective ${ }^{147} »$. Certes, il fallait « représenter » la jeunesse et se mettre à son écoute, mais seulement dans la mesure où elle comprendrait qu'il lui fallait à tout prix éviter l'écueil de l'assimilation. Le relativisme démocratique, sur ce point, avait atteint sa limite. On s'appliqua très peu, pour le reste, à définir cette « conscience collective » dont on souhaitait si ardemment l'émergence. À une autre époque, l'Asfo, encore une fois, se serait évertuée à démontrer à la jeunesse en quoi elle appartenait à une minorité «nationale » qui faisait partie intégrante de l'un des « peuples fondateurs » du Canada et dont l'identité reposait sur le partage d'une langue, d'une culture, d'une foi et d'une mémoire communes avec l'ensemble des Canadiens français de l'Ontario, du Québec et d'ailleurs. Dans le contexte de 1968, la question «Qui sommes-nous ? » ne semblait plus exiger de réponse aussi précise...

Quoi qu'il en fût, l'Association provinciale des mouvements de jeunes de l'Ontario français fut mise à l'essai pendant un an et

146. [Roger Charbonneau], « Ottawa recevra la jeunesse franco-ontarienne dimanche prochain », [communiqué diffusé à la presse française de l'Ontario le $1^{\mathrm{er}}$ décembre 1949], CRCCF, FAJFO, C9/2/1 (souligné dans l'original).

147. " Procès-verbal. II ${ }^{\mathrm{e}}$ Conférence-Consultation de la jeunesse francoontarienne, 18, 19 et 20 mai 1968 », p. 4, CRCCF, FAJFO, C9/9/4. 
demi ${ }^{148}$. En octobre 1969 eut lieu son premier congrès qui rassembla plus de 160 jeunes représentant 72 associations situées un peu partout en province, signe tangible de la réussite du projet $^{149}$. Entre-temps, cependant, l'APMJOF n'avait pas chômé, et c'est bien le moins que l'on puisse dire. L'une de ses premières démarches, par exemple, consista à demander à être représentée au sein de l'ACFÉo à titre de porte-parole officiel de la jeunesse franco-ontarienne ${ }^{150}$. Elle prépara également un mémoire pour le Comité franco-ontarien d'enquête culturelle que le gouvernement Robarts avait mis sur pied en mai 1967 et qui représentait une occasion inespérée pour l'APMJOF de se positionner comme la voix officielle, encore une fois, de la jeunesse francophone de l'Ontario $^{151}$. Elle profita de cette tribune pour réclamer un appui financier de la part des instances gouvernementales dans le dessein d'exécuter son programme d'animation culturelle à l'échelle provinciale. L'importance de l'APMJOF, non seulement pour la jeunesse, mais aussi pour tout l'Ontario français, ne devait faire aucun doute : «L'unité des jeunes [F]ranco-[O]ntariens se fera en autant que 1'A.P.M.J.O.F. est reconnue par les autorités gouvernementales. [...] L'avenir de la communauté francoontarienne sera assuré en autant que l'A.P.M.J.O.F. reçoit un appui

148. Notons que le nom «APMJOF » ne fut pas donné au mouvement au moment même de la deuxième CCJFO, mais plutôt lors de la première réunion du «Comité provincial », qui eut lieu le mois suivant à Ottawa («Procès-verbal de la première réunion du Comité provincial tenue les 15 et 16 juin 1968 à la Maison franco-ontarienne, Ottawa », CRCCF, FRB, P45/1/3).

149. Assemblée provinciale des mouvements de jeunes de l'Ontario français, « Premier congrès général. 11, 12 et 13 octobre 1969 », CrCCF, FAJFo, C9/37/4.

150. Lettre de Pierre-Yves Allard, du Comité provincial de l'Apmjof, à Roger Charbonneau, secrétaire de l'ACFÉo, 19 juin 1968, CRCCF, FrB, P45/1/3. L'ACFÉo accepta la requête de l'APMJOF, mais décida de l'affilier " unilatéralement », sans rencontrer ses dirigeants, qui en furent fortement offusqués. L'ApmJoF accepta la décision, mais lança un avertissement aux « aînés » : «Si la jeunesse ne peut participer aux décisions dans la mesure désirée, le Comité provincial de l'ApmJof se verra dans l'obligation de prendre la décision qui s'impose, décision catégorique s'il le faut » («Représentation à l'Association d'éducation », Bulletin de nouvelles [de l'Apmsof], janvier 1969, p. 2, CRCCF, FAJFO, C9/37/2).

151. «L'Origine, la situation actuelle et les perspectives d'avenir dans le domaine culturel de l'Assemblée provinciale des mouvements de jeunes de l'Ontario français », mémoire présenté au Comité franco-ontarien d'enquête culturelle, 1968, CrCCF, FrB, $\mathrm{P} 45 / 1 / 7$. 
politique et financier du gouvernement ontarien ${ }^{152}$. „) Voilà qui était clair : l'avenir de l'Ontario français était lié à celui de sa jeunesse qui, pour sa part, dépendait de l'Apmjof. L'année suivante, l'Association se mêla de la crise linguistique que venait de déclencher l'adoption, par le gouvernement unioniste de JeanJacques Bertrand, de la loi 63, qui garantissait aux contribuables québécois la liberté de choisir la langue dans laquelle leurs enfants recevraient leur instruction primaire. L'APMJOF monta aux barricades pour dénoncer cette mesure « aliénante » qui n'aurait eu d'autre conséquence que d'affaiblir la culture française au Québec et au Canada dans son ensemble ${ }^{153}$. Elle conclut, par ailleurs, une entente de principe avec la Fédération des centres culturels du Québec dans le dessein « d'établir un réseau complet de diffusion culturelle » en Ontario dans les domaines des arts visuels, de l'artisanat, du cinéma, de la musique, du théâtre, de la chanson, etc. ${ }^{154}$ L'APMJOF travailla également à la mise sur pied d'un « club du livre » qui vit le jour très rapidement en octobre 1968. Regroupant près de 700 membres dès sa première année, le club proposa à la jeunesse franco-ontarienne des titres allant des aventures de Tintin et d'Astérix aux ouvrages de Karl Marx, de Friedrich Engels et de Che Guevara, en passant par les œuvres de Claire Martin, d'Alphonse Daudet, de Pierre Mendès France et d'Émile Nelligan ${ }^{155}$.

Si l'Apmjof avait le vent dans les voiles, l'AJfo, pour sa part, avait du plomb dans l'aile. Déjà, en février 1968, l'administrateur du Bistro (et ancien secrétaire général de l'AJfo), Jean-Claude Carisse, réclama qu'on lui cédât l'ensemble de l'équipement de bureau de l'AJfo qui songeait, rappelons-le, à déplacer ses

152. Ibid., p. 4.

153. Paul-François Sylvestre, secrétaire général de l'APMJOF, « Communiqué de l'Apmjof. Le bill 63 au Québec », s.d., CRCCF, FrB, P/45/1/9.

154. "Accord de principe entre l'Association provinciale des mouvements de jeunesse de l'Ontario français et la Fédération des centres culturels de la province de Québec », communiqué de presse, 19 mars 1969, CRCCF, FrB, P45/1/9.

155. «Premier rapport annuel du "Club du livre" de l'ApMJOF [1969 ?] », CRCCF, FAJFO, C $9 / 37 / 7$. 
quartiers généraux vers Welland ${ }^{156}$. Carisse exigea aussi que l'AJFO renonçât entièrement à sa part des profits du tirage annuel, qui devaient plutôt être partagés également entre le Bistro et la CCJFO. Il ne faisait aucun doute, aux yeux de Carisse, que le déménagement du Bureau central vers le Sud - qui n'aurait pas lieu, en fin de compte - marquerait le « début de la fin » de l'AJfo. En novembre 1968, l'Association ne comptait plus que six cercles, dont cinq dans le Sud ${ }^{157}$. Le cercle de Welland, pour sa part, avait même rejoint les rangs de l'ApMJOF, sans toutefois abandonner son affiliation à l'AJfo. Pour le reste, la cohabitation des deux organismes, AJFo et APMJOF, à l'intérieur des mêmes bureaux à la Maison franco-ontarienne à Ottawa devenait de plus en plus problématique. La première en vint graduellement à voir dans la seconde un regroupement « radical» qui l'empêchait de fonctionner normalement ${ }^{158}$. Le conflit prit une allure cocasse lorsqu'en juin 1969, l'Ajfo exigea de 1'«Union radicale d'Ontario » qu'elle quittât les lieux sur-le-champ :

Le Conseil provincial [l'ancien Bureau central] de l'Association de la jeunesse franco-ontarienne [...] demande aujourd'hui à l'[Union radicale d'Ontario], ce mouvement fantôme qui se vante d'avoir contrôlé la conférence[-]consultation de la jeunesse franco-ontarienne et de contrôler maintenant l'APMJOF, de bien vouloir quitter le bureau de l'AJfo avant le 17 juillet prochain. Depuis plus d'un an, le spectre révolutionnaire de votre organisme hante notre local et la réévaluation de ce bureau nous force aujourd'hui de demander le déménagement de l'URO, ses affiches et ses odeurs... ${ }^{159}$

156. Lettre de Jean-Claude Carisse, administrateur du Bistro, au Bureau central de l'Ajfo, 21 février 1968, CRCCF, FrB, P45/1/16. Notons que Carisse venait tout juste de démissionner du Bureau central de l'AJfo ( ( Procès-verbal de la réunion du Bureau central de l'Association de la jeunesse franco-ontarienne tenue lundi le 12 février 1963 à la Maison franco-ontariennne », CRCCF, FAJFO, C9/12/6.

157. « Procès-verbal de la réunion du comité provincial [de l'AJfo] le 10 novembre 1968, à Toronto », CRCCF, FAJFO, C9/12/6.

158. « Procès-verbal de la réunion du comité provincial de l'Association de la jeunesse franco-ontarienne tenue le 19 mars 1969 à Ottawa », CRCCF, FAJFO, C9/12/7.

159. Lettre de Gérard Lévesque, du Conseil provincial de l'Ajfo, «À qui de droit », 17 juin 1969, CRCCF, FRB, P45/10/2. 
Cet extrait laisse entrevoir que le conflit entre l'AJFo et l'APMJOF ne tenait pas qu'à une simple question de partage de ressources : il possédait également des sources idéologiques plus profondes. La nouvelle Association adhérait corps et âme à l'idéologie de participation qui était en passe de balayer, au même moment, la jeunesse du monde occidental ${ }^{160}$. Dans la deuxième livraison du Bulletin de nouvelles de l'ApmJof, publiée en janvier 1969, le secrétaire général, Paul-François Sylvestre, y alla d'une réflexion fort marxisante sur le rôle de la jeunesse en Ontario français :

En 1969, la jeunesse de l'Ontario français doit se définir en un seul mot : avant-garde. Nous, camarades, nous devons être à l'avant-garde de tous les mouvements. Les premiers dans le travail. Les premiers dans l'étude. Les premiers dans la manifestation d'un Ontario français.

Camarades, 1969 doit être une année où la jeunesse ouvrière, rurale et étudiante pourra accéder à un mieux[-]être culturel, une année où chacun collaborera à la francophonie en Ontario. C'est là une tâche difficile et souvent ardue. Pour réussir, il nous faut prendre les outils nécessaires : la démocratie, la représentation et l'organisation.

Qu'il soit permis de formuler un souhait pour 1969 : une année où partout le jeune sera à l'avant-garde ${ }^{161}$.

Pourtant, 1'AJfo avait cheminé, elle aussi, sur la voie de la contreculture et de la «participation ». En 1970, elle présenta un mémoire à la Commission d'étude sur les laïcs et l'Église que l'épiscopat canadien-français avait mise sur pied, en 1968, au lendemain du concile Vatican II et que présidait le sociologue Fernand Dumont. Les signataires ne cherchèrent pas à discréditer l'Église et le catholicisme, mais ils n'en soulevèrent pas moins le

160. Les historiens et sociologues ont déjà étudié l'impact de l'idéologie de la participation (et du marxisme) sur la jeunesse québécoise au tournant des années 1970. Voir, entre autres : Madeleine Gauthier, « Le Mouvement étudiant des années soixante comme aspect du mythe de la Révolution tranquille? », op. cit., ; Éric Bédard, Chronique d'une insurrection appréhendée. La crise d'Octobre et le milieu universitaire, Sillery, Septentrion, 1998, 199 p. ; Jean-Philippe Warren, Ils voulaient changer le monde. Le militantisme marxiste-léniniste au Québec, Montréal, vLB éditeur, 2007, 252 p.

161. Paul-François Sylvestre, «Une nouvelle année », Bulletin de nouvelles [de l'Apmjof], janvier 1969, p. 1, CRCCF, FAJFO, C9/37/2. 
problème de la désaffection religieuse au sein de la jeunesse qui cherchait, de son côté, à « faire de l'authenticité, de la liberté, de l'amitié vraie (valeurs universellement et profondément ancrées chez tous les jeunes) les bases d'une religion renouvelée, pour ne pas dire nouvelle ${ }^{162} »$. Il arrivait, poursuivit-on, que les « curés » et les autres « personnes constituées en autorité » ne fissent aucune distinction entre « pouvoir » et « autorité ». Cette dernière, « au lieu de venir d'en haut », devait être « partagée » afin de rendre plus représentative et démocratique l'institution ecclésiastique, qui était menacée, sinon, de crouler sous le poids de son inertie et de perdre à jamais la jeunesse :

Les jeunes recherchent l'authenticité. Or, les formes actuelles de l'Église ne leur permettent pas d'exprimer leurs sentiments et leurs idées à leur façon. C'est ce qui en amène plusieurs à refuser totalement l'Église. Ou alors, avec la même sincérité, d'autres veulent adapter les cadres ou en créer de nouveaux. Ils se donnent donc leur propre liturgie tout en se disant que les formes traditionnelles pourront toujours coexister et servir à d'autres ${ }^{163}$.

Que l'AJFo ait décidé d'intervenir dans le débat sur l'avenir de l'Église canadienne-française témoigne peut-être, en dépit de son évolution idéologique récente, d'un reste d'attachement vis-àvis de la chose religieuse. L'APMJOF, de son côté, ne prêta aucune attention à la réforme de l'Église. En définitive, elle avait adopté une attitude beaucoup plus intransigeante sur plusieurs questions chaudement débattues dans le contexte de la fin des années 1960. Nous avons mentionné, ci-dessus, la critique qu'elle fit du projet de loi 63. Dans son journal mensuel, Franc-Jeu, l'AJfo reprit à son compte un éditorial de Marcel Gingras, du Droit, qui voyait dans cette prise de position de l'ApmJof un écart de conduite inacceptable, un « manque de réalisme » flagrant, une « aberration pure et simple » et un « illogisme incommensurable ${ }^{164} »$.

162. «Mémoire de l'AJfo à la Commission Dumont ", Franc-Jeu, juin-juillet 1970, p. 1, CRCCF, FAJFO, C9/36/8. Les parenthèses sont des auteurs du mémoire.

163. Ibid., p. 1.

164. Marcel Gingras, « Un manque de réalisme [article repris du Droit] », FrancJeu, novembre 1969, p. 3, CRCCF, FAJFO, C9/36/8. 
Comment l'APMJOF pouvait-elle prêcher l'unilinguisme français au Québec tout en favorisant 1'épanouissement culturel de la minorité franco-ontarienne - et donc le bilinguisme en Ontario ? Ne venait-elle pas tout juste de recevoir elle-même une subvention substantielle de son propre gouvernement provincial « en faveur de la cause française en Ontario » ? Le Secrétariat d'État n'avaitil pas contribué généreusement, lui aussi, à l'organisation de ses colloques et de ses rencontres ? La nouvelle Association avait mieux à faire pour favoriser le progrès de la communauté francoontarienne, ajouta Gingras, que de s'en prendre à la minorité anglo-québécoise : « Il y a encore tellement à faire en Ontario que l'APMJOF n'aura pas assez d'une génération pour consolider l'œuvre de ses aînés. Qu'elle se donne donc à la tâche qui lui est propre. Elle contribuera ainsi à la gloire du Canada français et de son noyau principal, le Québec ${ }^{165}$. »

Il semble que l'ApMJOF n'ait pas apprécié la critique que lui adressa 1'AJfo par la plume de Gingras ${ }^{166}$. Dans la livraison suivante de Franc-Jeu, le rédacteur en chef, Jacques de Courville Nicol, se livra à une dénonciation en règle de l'ApMJOF, qu'il accusa carrément de censure : les exemplaires de Franc-Jeu destinés au Collège Glendon de l'Université York de Toronto où étudiait le président de l'APMJOF, Jean-François Aubé - avaient été retournés sans cérémonie à Ottawa ${ }^{167}$. La position de Gingras avait été fort critique à l'endroit de l'APMJof, admit de Courville Nicol, mais il ne fallait pas l'imputer à l'AJfo. Le nœud du problème, de toute manière, se situait ailleurs. Franc-Jeu avait sondé ses lecteurs pour connaître leur opinion face au projet de loi 63 et la très grande majorité des répondants s'était opposée à l'unilinguisme français au Québec. De quel droit l'APMJOF osaitelle se prononcer dans ce dossier alors que sa position ne reflétait pas, vraisemblablement, celle de la masse des jeunes Franco-

165. Ibid., p. 3 .

166. Dans la même livraison du journal, l'équipe éditoriale de Franc-Jeu reproduisit un article semblable de Hugues Albert du Voyageur de Sudbury.

167. Jacques de Courville Nicol, « Démocratie d'un seul au Collège Glendon », Franc-Jeu, édition spéciale 1969-1970, p. 2, CRCCF, FAJFO, C9/36/8. 
Ontariens ? Où étaient la représentativité, la liberté d'expression et la démocratie, dans tout cela ? « Nous ne pouvons laisser passer un tel affront aux collaborateurs du journal, aux membres de l'AJfo, aux tenants d'une démocratie saine et efficace, renchérit de Courville Nicol. [N]ous ne pouvons tolérer que la démocratie d'un seul au collège Glendon passe inaperçue ! » Le journal offrit même aux associations membres de l'ApMJof de leur ouvrir ses pages afin qu'elles pussent exprimer librement et directement leur opinion sur le bien-fondé de la loi 63 et sur les agissements de leur président, Jean-François Aubé. Ce dernier avait bien senti qu'il avait peut-être commis une imprudence en condamnant aussi vertement le bilinguisme scolaire au Québec. Dans la même livraison, Franc-Jeu reprit un autre article du Droit dans lequel Aubé tenta, du mieux qu'il put, de tirer son épingle du jeu : l'intention de l'ApmJof n'avait jamais été d'appuyer l'unilinguisme, mais plutôt de favoriser le développement de la culture française au Québec ${ }^{168}$. Il ne s'agissait, autrement dit, que d'un triste malentendu entraîné par la formulation imprécise du communiqué de presse à l'origine de la controverse. Les explications du président de l'ApmJof étaient cependant peu convaincantes, le communiqué ayant carrément présenté la loi 63 comme une mesure « aliénante » qui aurait «affaibli » la culture française non seulement au Québec, mais dans l'ensemble du Canada.

L'année suivante, la question du bilinguisme pousserait les deux associations à croiser le fer de nouveau. Cette fois, le champ de bataille serait le milieu universitaire. Au début de 1970, l'AJFO annonça la mise sur pied d'une « commission d'enquête » sur le bilinguisme à l'Université d'Ottawa, qui venait elle-même de créer un groupe de travail sur la question linguistique ${ }^{169}$. L'Association envisagea de répéter l'expérience dans un avenir rapproché à

168. Solange Plourde-Chagnon, « Opposition de l'Apmuof à l'unilinguisme français [article repris du Droit] », Franc-Jeu, édition spéciale 1969-1970, p. 2, CRCCF, FAJFO, C9/36/8.

169. «L'AJFo crée une commission d'enquête sur le bilinguisme à l'Université d'Ottawa », Franc-Jeu, édition spéciale 1969-1970, p. 1, CRCCF, FAJFO, C9/36/8. 
l'Université Laurentienne de Sudbury et au Collège Glendon, les deux autres institutions universitaires bilingues de la province ${ }^{170}$. La commission de l'AJFo produisit un rapport qui rejeta catégoriquement toute velléité d'imposer l'unilinguisme français dans l'ancienne université oblate, quoiqu'elle proposât que cette dernière donnât immédiatement la «priorité » à la langue française ${ }^{171}$. L'unilinguisme aurait été une solution des plus " rétrogrades » et "néfaste[s] » pour la jeunesse francoontarienne. Il était impératif que les étudiants francophones apprissent l'anglais afin de « soutenir la concurrence » des travailleurs anglophones, de " communiquer efficacement » avec eux et de prendre une part active à la vie politique de la province. L'unilinguisme risquait de provoquer « de grands torts et de grandes injustices » dans une société qui s'orientait de plus en plus vers un « rapprochement de nos deux cultures et de nos deux langues fondatrices ${ }^{172} »$.

À l'ApMJOF, où l'on prêchait plutôt la francisation totale de l'université (comme à l'ACFO, d'ailleurs ${ }^{173}$ ), on en vint à la conclusion que la guerre intestine que se livrait la jeunesse francoontarienne par associations interposées devait prendre fin, coûte que coûte, d'autant plus qu'il semblait y avoir, de plus en plus, un dédoublement d'activités entre les deux organismes ${ }^{174}$. À son congrès de mai 1968, l'AJFo avait effectivement décidé, dans un esprit de « démocratisation », d'offrir des « services » culturels et sportifs à la jeunesse franco-ontarienne, surtout en matière de livres, de films, de musique, d'arts visuels, etc. ${ }^{175}$ Cette démarche

170. Ces enquêtes ne semblent pas avoir eu lieu.

171. «Priorité à la langue française à l'Université d'Ottawa, mais... opposition à l'unilinguisme ! ", Franc-Jeu, février-mars 1970, p. 1, 4, CRCCF, FAJFO, C9/36/8.

172. Ibid., p. 4.

173. «Mémoire présenté au groupe de travail sur le bilinguisme à l'Université d'Ottawa par l'Association canadienne-française de l'Ontario », 12 p., [probablement 1970], CRCCF, FAJFO, C9/40/5.

174. Jean-François Aubé, président du Conseil provincial de l’ApMJOF, « APMJOF, AJFo et la jeunesse », document annexé à une lettre circulaire d'Aubé, 10 avril 1970, CRCCF, FAJFO, C9/37/4.

175. Lorraine M.-A. Messier, Conseil provincial de l'AJfo, document daté du 29 octobre 1969, CRCCF, FAJFO, C9-2/1/6. 
ne recoupait-elle pas précisément une partie du mandat de l'Apmjof ? En octobre 1969, cette dernière reçut $24000 \$$ du gouvernement provincial destinés à l'embauche de trois animateurs culturels ${ }^{176}$. La manne gouvernementale tomberait également sur 1'AJfo, toutefois, ce qui aurait pour effet d'approfondir la rivalité entre les deux associations ${ }^{177}$. Par exemple, toujours en octobre 1969, au moment même où l'APMJOF recevait la subvention du gouvernement ontarien, l'AJfo fit paraître la première livraison du «nouveau » Franc-Jeu, qui venait d'entreprendre une véritable cure de beauté grâce aux bons soins de l'État. Depuis la parution de son premier numéro, deux ans et demi plus tôt, la feuille de l'AJfo n'avait été guère plus qu'un bulletin d'informations polycopié aux allures bien modestes et artisanales. L'appui financier des gouvernements lui permit, d'abord, de faire passer son tirage de 1000 à 25000 et, ensuite, d'avoir recours aux services d'un imprimeur professionnel - les presses du Carillon de Hawkesbury, en l'occurrence ${ }^{178}$. L'AJFO put également embaucher son premier administrateur permanent,

176. «Subvention à l’APMJOF », [probablement 1970], CRCCF, FAJfo, C9/37/5. L'APMJOF avait soumis un mémoire au Service des Loisirs et de la Jeunesse du ministère de l'Éducation de l'Ontario («L'Animation socio-culturelle auprès des jeunes de l'Ontario français ", mémoire au Service de la [J]eunesse et des [L]oisirs, ministère de l'Éducation ", [probablement 1969], CRCCF, Fonds Association provinciale des mouvements de jeunes de l'Ontario français (dorénavant FAPMJOF), C13-1/1/5). Soulignons que l'APMJOF avait effectué cette démarche conjointement avec l'AcFo, qui souhaitait obtenir, pour sa part, le financement nécessaire à l'embauche d'une vingtaine d'animateurs culturels (lettre de Roger Charbonneau, secrétaire général de l'ACFO, et de Paul-François Sylvestre, secrétaire général de l'APMJOF, à Louis-Philippe Poirier, surintendant adjoint, Service de la Jeunesse et des Loisirs du ministère de l'Éducation de l'Ontario, 4 décembre 1969, CRCCF, FAJFo, C9/37/5).

177. En juin 1969, l’AJfo rédigea un mémoire pour obtenir un appui financier du Secrétariat d'État ( "Mémoire de l'Association de la jeunesse franco-ontarienne », 13 juin 1969, 8 pages, CRCCF, FAJFO, C9-2/1/6). Elle obtint une somme de 5000 \$, en plus d'un montant de 1500 \$ qu'elle devait partager avec l'APMJof pour la mise en œuvre d'un programme conjoint d'animation culturelle. Nous y reviendrons (lettre de Gérard Pelletier, secrétaire d'État du Canada, à Rodrigue Landriault, président de l'AJFo, 15 août 1969, CRCCF, FAJFO, C9/37/5).

178. Jacques de Courville Nicol, « 25000 fois plus », Franc-Jeu, octobre 1969, p. 2, CrccF, FaJfo, C9/36/8. Le tirage de Franc-Jeu passerait à 30000 dès le mois suivant. 
Gérard Lévesque, et son propre animateur, Roland Dubue ${ }^{179}$. L'intervention financière des gouvernements réussit à ragaillardir l'Association qui proposa à la jeunesse, par l'intermédiaire de Franc-Jeu, un programme de « services » culturels et sportifs très développé ${ }^{180}$. L'Association comptait maintenant 21 représentants régionaux (à Toronto, à Kapuskasing, à Kirkland-Lake, à Sudbury, à Ottawa, à Alexandria et à Saint-Joachim) et dix cercles situés à Ottawa, à Cornwall, à Oshawa, à Toronto, à Welland, à Hamilton, à Sainte-Catherine, à Peterborough, à Cochrane et à Hearst ${ }^{181}$. Elle s'enorgueillit, par ailleurs, d'être la seule association de jeunesse francophone à posséder une charte provinciale, charte qu'elle avait obtenue dès sa fondation en 1949. Le temps de la « survivance » était révolu, clama l'AJfo, il fallait désormais mettre le cap sur la « croissance et l'expansion ${ }^{182}$ ».

L'AJFo avait effectivement repris du poil de la bête. Pourtant, le rédacteur en chef de Franc-Jeu sentit le besoin de démontrer à ses lecteurs que l'Association n'avait rien perdu de sa pertinence ni de son utilité. Si la fin des années 1960 était marquée par l' « insatisfaction » et la « contestation », Jacques de Courville Nicol était d'avis qu'il fallait modérer l'impulsion qui conduisait plusieurs jeunes à fuir vers l'avant, à vouloir « à tout prix faire du présent l'avenir » :

À tort ou à raison, un certain nombre de nos jeunes désirent pour corriger les inefficacités du passé et du présent, tout détruire ce qui existe aujourd'hui, afin de tout reconstruire dans du neuf pour l'avenir. Il faut savoir par ailleurs quand s'arrêter, car le passé conserve des richesses qu'il ne faut tout de même

179. «L'AJFO a son premier administrateur permanent », Franc-Jeu, octobre 1969, p. 1, CRCCF, FAJfo, C9/36/8; « Notre animateur AJFo!», Franc-Jeu, janvier-février 1970, p. 1, CRCCF, FAJFO, C9/36/8.

180. «Les Services de l'AJfo ... des réponses à des besoins !! », Franc-Jeu, octobre 1969, p. 3, CRCCF, FAJFO, C9/36/8.

181. «Qu'est-ce que l'AJfO ? ", Franc-Jeu, octobre 1969, p. 1, 3, CRCCF, FAJFO, C9/36/8; « 21 jeunes représentent nos sept régions », Franc-Jeu, octobre 1969, p. 2, CRCCF, FAJFO, C9/36/9.

182. «Qu'est-ce que l'AJfo ? ", Franc-Jeu, octobre 1969, p. 1, 3, CRCCF, FAJfO, C9/36/8. 
pas complètement détruire sans quoi on pourrait facilement se retrouver à l'âge de la pierre ${ }^{183}$ !

Si de Courville Nicol se livra à de telles cogitations sur l'esprit de la jeunesse contestataire, c'est qu'il existait, en Ontario, «plusieurs groupes de jeunes francophones qui prêch[ai]ent le limogeage de l'AJFO », qui avaient « émis contre elle un mandat d'arrêt de mort » et qui avaient décrété, sans autre forme de procès, qu'elle était « dépassée », qu'elle ne répondait plus aux besoins des jeunes et qu'elle devait céder la place « aux autres ». D'après lui, on ne pouvait pourtant pas détruire « une vieille association » comme on aurait démoli « un vieil édifice » : « une association, c'est presque humain, ça [sic] a des souvenirs, des allégeances, une histoire, un passé, des attaches ; ça [sic] a une façon de vivre, de penser, travailler et de croire ». L'AJFo qui, en 1969, célébrait son vingtième anniversaire, était donc le dépositaire d'une sagesse et - lâchons le mot - d'une tradition que seule l'expérience du passé pouvait engendrer. La jeunesse franco-ontarienne pouvaitelle renoncer à de telles assises, se demanda de Courville Nicol, sans courir le risque de se retrouver entièrement déboussolée ${ }^{184}$ ? À l'inverse, rien dans le discours de l'ApMjof ne permettait de supposer que ses membres - ou, du moins, ses dirigeants valorisaient la tradition et les enseignements du passé de la même manière. Au contraire, la tradition était présentée, de manière générale, comme un obstacle dont il aurait plutôt fallu s'affranchir afin de rendre possible - enfin ! - l'épanouissement plein et entier de la jeunesse franco-ontarienne. Bien que son évolution idéologique eût été considérable, comme nous avons pu le constater, depuis le début de la décennie, l'AJFo conservait, malgré tout, quelques vestiges de conservatisme qui l'empêchaient

183. Jacques de Courville Nicol, « La Tribune éditoriale », Franc-Jeu, novembre 1969, p. 2, CRCCF, FAJFO, C9/36/8.

184. Au moment de sa démission de la rédaction de Franc-Jeu, un an plus tard, de Courville Nicol se décrirait comme le « DÉFENSEUR de l'ordre établi, des structures existantes et de la continuité », et comme le «PROMOTEUR de la démocratie, du droit au libre choix de l'individu, de l'unification des jeunes Franco-[O]ntariens ». Voir Jacques de Courville Nicol, « Un avant[-]dernier mot... », Franc-Jeu, octobrenovembre 1970, p. 2, CRCCF, FAJFO, C9/36/8. 
d'emboîter le pas à l'APMJOF dans son rejet quasi total du traditionalisme canadien-français.

\section{Direction-Jeunesse (DJ)}

La rivalité entre les deux associations pouvait-elle se prolonger indéfiniment? La jeunesse franco-ontarienne était-elle suffisamment nombreuse pour soutenir deux organismes rivaux qui se disputaient son allégeance avec une âpreté croissante ? Les bailleurs de fonds gouvernementaux, dont dépendaient de plus en plus l'APMJOF et l'AJFO, continueraient-ils pendant encore très longtemps de financer pareille rivalité ? L'année 1969-1970 serait déterminante à cet égard et contraindrait les deux mouvements à faire, de part et d'autre, des compromis. Pourtant, l'AJFo n'avait donné aucun signe qu'elle envisageait de jeter l'éponge, bien au contraire. Le 17 janvier 1970, elle organisa avec grand faste un banquet pour célébrer son vingtième anniversaire. Parmi les invités d'honneur, on compta, entre autres, l'ambassadeur de France à Ottawa et Fernand Guindon, ministre du gouvernement Robarts. L'allocution de circonstance fut prononcée par le député de Nickel-Belt à la Législature ontarienne, Gaston Demers, luimême un ancien membre de l'AJfo. Dans son compte rendu de la soirée, Franc-Jeu rapporta que le député conservateur avait appuyé sans réserve les efforts de l'Association et avait prévenu l'assemblée « qu'il ne fallait pas que l'AJfo et son œuvre se [vissent] défaites par d'autres groupes qui [voulaient] la supplanter ${ }^{185}$ ». Demers enchaîna en critiquant la propension des Franco-Ontariens à se dédoubler dans leurs démarches, ce qui leur coûtait en fin de compte très cher en temps, énergie et ressources. Il termina son allocution en exhortant la jeunesse franco-ontarienne à conquérir le domaine économique en faisant preuve d'autant de cran que les grands héros du Canada français, dont Pierre-Esprit Radisson, Henri Bourassa et, plus récemment,

185. " "Si nous avions $\$ 100.00$ pour chaque effort perdu à la duplication nous serions un peuple très riche..." ", Franc-Jeu, janvier-février 1970, p. 1, CRCCF, FAJFO, C9/36/8. 
Paul Desmarais et Robert Campeau, tout en maintenant la « bonne entente » qui unissait les Franco-Ontariens à leurs « frères » de l'Ontario et du Canada dans son ensemble ${ }^{186}$.

Cet appui à l'AJFO - appui imprégné de «bon-ententisme » et de traditionalisme canadien-français - eut sûrement l'heur de donner des munitions à l'Association dans le conflit qui l'opposait à 1'Apmjof. D'ailleurs, l'administrateur de l'AJfo, Gérard Lévesque, expédia sur-le-champ un communiqué de presse pour rendre public ce qu'il présenta implicitement comme la position officielle du gouvernement ontarien sur la jeunesse francoontarienne ${ }^{187}$. L'allocution de Demers fut cependant suivie de quelques échanges assez corsés avec l'assemblée au cours desquels il dut, à l'aide du ministre Guindon, rendre compte de la position de son gouvernement vis-à-vis du conflit entre les deux mouvements de jeunesse. Était-il vrai que le Service des Loisirs et de la Jeunesse du ministère de l'Éducation favorisait discrètement, mais concrètement, l'APMJOF, aux dépens de l'AJFo ? Dans l'affirmative, n'était-il pas dangereux et, surtout, illégitime « de mettre à l'emploi d'un certain corps intermédiaire (l'APMJOF) des fonctionnaires de l'État [les animateurs culturels], au détriment [...] d'un autre groupe ou corps intermédiaire (l'AJfo) qui, lui, exist[ait] depuis plus de [vingt] ans et a[vait] déjà fait plus que ses preuves ${ }^{188}$ » ? Demers et Guindon répondirent qu'il n'avait jamais été question de favoriser qui que ce fût et que les subventions gouvernementales avaient été mises à la disposition de l'ensemble de la jeunesse franco-ontarienne, sans discrimination. Le ministre indiqua tout de même son intention de se pencher sur la question afin d'en avoir le cœur net.

Y avait-il anguille sous roche? L'Apmjof et le Service des Loisirs et de la Jeunesse paraissaient entretenir des relations fort cordiales, en effet, comme en témoigne la correspondance entre

186. Ibid., p. 1.

187. Communiqué de presse de Gérard Lévesque, administrateur de l'Asfo, 17 janvier 1970, CRCCF, FAPMJOF, C13-1/1/6.

188. " "Si nous avions $\$ 100.00$ pour chaque effort perdu à la duplication nous serions un peuple très riche..." ", Franc-Jeu, janvier-février 1970, p. 1, CRCCF, FAJFO, C9/36/8. Les parenthèses sont de l'auteur de l'article. 
le secrétaire général de l'Association, Paul-François Sylvestre, et le surintendant adjoint du Service, Louis-Philippe Poirier, luimême un ancien proche de $1^{\prime} A J f O^{189}$. Après avoir été mis au courant du bruit que cette affaire avait fait dans Le Droit, Poirier prit l'initiative de s'expliquer directement auprès du ministre Guindon. Il était vrai que le Service des Loisirs et de la Jeunesse avait accordé à l'ApMJOF une subvention de plus de 20000 \$ et qu'il s'était engagé à lui verser une somme annuelle allant jusqu'à $30000 \$$ sur les trois prochaines années, lui confirma-t-il. De tels montants étaient effectivement substantiels, mais il était impossible que le gouvernement ontarien considérât l'APMJOF tout simplement comme un « groupe particulier ». Il s'agissait plutôt d'une " association "parapluie" » qui regroupait de nombreux organismes régionaux et provinciaux, dont l'AJFO. Cette dernière avait même parrainé la Conférence-consultation de la jeunesse franco-ontarienne qui avait donné naissance à l'APMJOF, deux ans plus tôt. Sous les auspices de l'AJFo, la nouvelle association avait reçu de ses membres un mandat sans équivoque.

C'est donc dire, précisa Poirier, que par le truchement de l'APMJOF, tous les mouvements [f]ranco-[o]ntariens peuvent bénéficier des services généraux et particuliers de l'ApMJOF de même que ceux [de ses] animateurs. Ces animateurs, tel qu'entendu, travaillent dans l'optique de l'APMJOF, c'est-à-dire que l'orientation de leur travail doit être en ligne avec les buts et objets $[$ sic $]$ généraux de l'ApMJOF. [...] [I]l restera $[\ldots]$ à l'ApMJOF d'identifier les besoins communs de toute la jeunesse Franco Ontarienne [sic] et de situer et promouvoir des activités d'ensemble qui devront répondre aux besoins généraux ${ }^{190}$.

Autrement dit, l'APMJOF n'était pas une association comme les autres. Elle était, en réalité, l'organisme le plus clairement autorisé à parler au nom de l'ensemble de la jeunesse franco-ontarienne

189. Échange de correspondances entre Paul-François Sylvestre, secrétaire général de l'ApmJof, et Louis-Philippe Poirier, surintendant adjoint du Service de la Jeunesse et des Loisirs, ministère de l'Éducation de l'Ontario, 14 et 16 janvier 1970, CRCCF, FAJFO, C9/37/5.

190. Lettre de Louis-Philippe Poirier à Fernand Guindon, ministre d'État du gouvernement ontarien, 23 janvier 1970, CRCCF, FAJFO, C9/37/5. 
et de ses mouvements. L'AJFo n'était rien de plus qu'une composante de 1'organisme-parapluie qu'était 1'APMJOF, organisme qu'elle avait elle-même contribué - et puissamment - à mettre sur pied. De quoi se plaignait-elle, alors ? La guerre froide entre les deux associations avait assez duré, de renchérir Poirier, qui avait également expédié cette lettre en copie conforme au secrétaire général de l'A PMJOF, Paul-François Sylvestre, à l'administrateur de l'AJfo, Gérard Lévesque, au secrétaire général de l'AcFo, Roger Charbonneau, et au député Gaston Demers. Il fallait, en dernière analyse, que la jeunesse prît les mesures nécessaires pour mettre un terme au conflit qui la divisait : « À ce moment-ci, il est de la responsabilité de l'APMJOF, de l'AJfo et de tous les autres mouvements de se rencontrer, de discuter de leurs problèmes respectifs, de trouver des solutions et de venir à une entente afin qu'il n'y ait pas dédoublement d'efforts et de services $^{191} »$. À la lumière de ces précisions, le ministre Guindon signifia à Poirier qu'il souhaitait, lui aussi, que la jeunesse francoontarienne réussît à «serrer les rangs » afin de dissiper cette « situation pour le moins nébuleuse », ne fût-ce que pour éviter que, devant tant d'incertitude, l'appui financier du gouvernement ontarien ne leur fût retiré ${ }^{192}$. Les bailleurs de fonds avaient parlé.

Malgré le conflit qui les divisait, l'AJfo et l'ApMJof avaient déjà entrepris des démarches en vue d'une éventuelle réconciliation. À l'automne 1969, elles avaient reçu du Secrétariat d'État une modeste subvention de 1500 \$ pour l'organisation d'un stage conjoint de formation en animation sociale ${ }^{193}$. Mais le temps des grands moyens était venu. Lors du premier congrès annuel de l'APMJOF, en octobre 1969, les délégués avaient proposé que les services des deux mouvements fussent carrément

191. Ibid.

192. Lettre de Fernand Guindon, ministre d'État du gouvernement ontarien, à Louis-Philippe Poirier, surintendant adjoint du Service de la Jeunesse et des Loisirs, ministère de l'Éducation de l'Ontario, 5 février 1970, CrCCF, FAJFO, C9/37/5.

193. Lettre de Gérard Pelletier, secrétaire d'État du Canada, à Rodrigue Landriault, président de l'AJfo, 15 août 1969, CRCCF, FAJfo, C9/37/5 ; lettre de Guy Voisin, du Secrétariat d'État, à Rodrigue Landriault, 18 septembre 1969, CRCCF, FAJFO, C9/37/5. 
fusionnés ${ }^{194}$. Aucune démarche bien concrète n'avait été effectuée à ce moment, mais au lendemain de l'intervention de Gaston Demers au banquet du vingtième anniversaire de l'AJfo, la réalisation du projet parut soudainement beaucoup plus urgente. Ainsi, le comité exécutif de l'APMJOF eut une discussion franche et ouverte pour trouver une solution aux problèmes qui minaient les relations entre les deux associations ${ }^{195}$. Le président, JeanFrançois Aubé, reconnut que les gouvernements et les organismes subventionnaires comprenaient mal que deux associations aussi similaires pussent prétendre représenter l'ensemble de la jeunesse franco-ontarienne, dédoublant ainsi les services et, surtout, les demandes de financement. Il fallait donc faire front commun, pour le plus grand bien de la jeunesse. Les autres membres du comité exécutif partagèrent le sentiment de leur président et se rallièrent à l'idée d'organiser un congrès pour préparer la fusion plus ou moins complète des deux organismes, pour peu que l'AJFO acceptât, bien entendu, de marcher avec sa rivale.

Lors d'une conférence de presse organisée conjointement avec l'Apmjof, l'exécutif de l'AJfo annonça qu'il accordait son aval au projet ${ }^{196}$. Il fut décidé qu'un congrès aurait lieu en mai 1970 dans le dessein de trouver une solution à leur différend et d'étudier la possibilité de mettre sur pied une nouvelle structure qui pût mieux répondre aux besoins de la jeunesse franco-ontarienne et faciliter la collaboration entre les deux organismes ${ }^{197}$. Les dirigeants des deux mouvements indiquèrent qu'ils se soumettraient à la volonté de l'assemblée dans la mesure où les résolu-

194. Assemblée provinciale des mouvements de jeunes de l'Ontario français, « Premier congrès général. 11, 12 et 13 octobre 1969 », CRCCF, FAJFO, C9/37/4.

195. « Procès-verbal de la réunion du comité exécutif de l'APMJoF, tenue à Ottawa, dimanche le 18 janvier $1970 »$, CRCCF, FAPMJOF, C13-1/1/2.

196. Les dirigeants des deux organismes s'étaient réunis à la fin janvier (PaulFrançois Sylvestre, « Rapport du secrétaire général » au Conseil provincial de l'APMJOF, 24 janvier 1970, CRCCF, FAPMJOF, C13-1/1/7).

197. «Avis important », Franc-Jeu, janvier-février 1970, p. 1, CRCCF, FAJfo, $\mathrm{C} 9 / 36 / 8$. Il faut souligner que les procès-verbaux des réunions du conseil provincial de l'AJFo se font de plus en plus rares, après 1968, dans les archives de l'Association. Heureusement, Franc-Jeu semble avoir rendu compte très minutieusement de la plupart des grandes décisions qui furent prises à l'AJFo pendant cette période. 
tions obtiendraient les deux tiers des voix exprimées. L'AJfo faisait preuve, toutefois, d'un enthousiasme plus que mitigé vis-à-vis du projet. Dans un article au titre évocateur paru dans Franc-Jeu, «L'Ajfo ? L'Apmjof ? L'Apjfo ? ou "Vox clamantis in deserto"? », Jacques de Courville Nicol dénonça l'ingratitude des pourfendeurs de l'AJfo qui l'avaient ni plus ni moins acculée au pied du mur ${ }^{198}$. Depuis vingt ans, l'AJfo avait travaillé inlassablement et bénévolement au service des jeunes FrancoOntariens. Certes, elle avait essuyé des échecs, mais ses réussites étaient nombreuses. Parmi les adversaires de l'Association, de Courville Nicol nomma, au premier chef, l'APMJof, qu'il accusa de fomenter sa perte afin de la supplanter une fois pour toutes. Il décocha également quelques flèches en direction de l'État ontarien, qui avait injustement soutenu, de ses deniers, la rivale de l'AJfo. Il s'en prit même au rédacteur en chef du Droit, Marcel Gingras. En effet, dans l'éditorial qu'il consacra au banquet d'anniversaire de l'AJFO, Gingras avait dit s'inquiéter, « avec [...] Gaston Demers, de la quasi-hostilité que [1'Association] manifest[ait] à l'endroit de l'[APMJOF] », et sommé l'AJfo de reconnaître qu'elle devait se voir non pas comme sa concurrente, mais plutôt comme l'un de ses « éléments constituants ${ }^{199}$ ». Pour de Courville Nicol, c'en était trop. Demers avait plutôt appuyé l'AJFo dans son conflit avec l'APMJOF, preuve que le rédacteur du Droit n'avait strictement rien compris à la situation : « On pourrait bien écrire à ce gentil journal pour en aviser le personnel de la rédaction que l'AJFo accepte de se laisser mordre de temps en temps et d'endurer, mais qu'il y a une limite entre se laisser mordre et se faire dévorer ${ }^{200}$. » Pourtant, il reconnut, dans le même souffle, qu'il n'existait plus d'autre recours que de baisser les bras devant l'inévitable, «la parole des tenants de notre AJFO » ayant été

198. Jacques de Courville Nicol, «L'AJfo ? L'Apmjof ? L'ApJfo ? ou "Vox clamantis in deserto"? ", Franc-Jeu, janvier-février 1970, p. 2, CRCCF, FAJfo, C9/36/8.

199. [Marcel Gingras], « Un vingtième anniversaire », Le Droit, 26 janvier 1970, article repris dans Franc-Jeu, janvier-février 1970, p. 2, CRCCF, FAJFO, C9/36/8.

200. Jacques de Courville Nicol, «L'Ajfo ? L'Apmjof? L'Apjfo ? ou "Vox clamantis in deserto"? », Franc-Jeu, janvier-février 1970, p. 2, CRCCF, FAJFO, C9/36/8. 
réduite à « la voix qui cri[ait] dans le désert ». De guerre lasse, l'Association s'était résignée à l'idée de mettre sur pied, de concert avec sa rivale, une « super-structure », pourvu que cette dernière fût assez «souple » pour permettre aux deux organismes de conserver leur identité propre :

L'heure d'une décision est arrivée et s'impose. Il faut ou s'entendre ou continuer à se battre... la dernière alternative [sic] me semble peu plausible. Les esprits sont peut-être échauffés, les allégeances durcies, les émotions trop grandes, les susceptibilités à fleur-de-peau... mais je suis sûr que l'enjeu - l'avenir de notre jeunesse francophone en Ontario - en vaut plus que la peine.

[...] Qu'il y ait divergences d'opinions ou d'idéalogie [sic] à l'intérieur d'une structure rénovée et incluant les deux associations provinciales actuelles est possible et même désirable... mais que ces divergences s'expriment autour d'une même table, à l'intérieur d'une même structure entre jeunes [O]ntariens d'une même appartenance linguistique et culturelle est urgent, essentiel et vital.

[...] [T] entons de nous donner une Association [p]rovinciale de [j]eunes [f]rancophones [o]ntariens (APJFO) au véritable sens du terme, en [n'] excluant personne ${ }^{201}$ !

Pour des raisons qui ne paraissent pas claires, le congrès prévu pour mai 1970 n'aurait lieu qu'en octobre. Selon l'ApmJof, le délai était toutefois imputable au manque d'engagement du Conseil provincial de l'AJFo envers le projet, hypothèse cependant impossible à vérifier ${ }^{202}$. Quoi qu'il en fût, les tergiversations de l'AJFO, si tergiversations il y eut, n'entamèrent aucunement la résolution de l'APMJOF, qui revint à la charge avec la publication d'un « livre blanc » sur le problème des mouvements de jeunesse. La jeunesse pouvait-elle se « payer le luxe » de deux organismes?, se demanda le président Aubé, qui en appela aux jeunes eux-

201. Ibid., p. 2.

202. Conseil provincial de l'Apmuof, « ApmJoF, AJfo et la jeunesse », document [«livre blanc»] annexé à une lettre circulaire de Jean-François Aubé, président de l'ApmJof, avril 1970, p. 2, CRCCF, FAJFO, C9/37/4. 
mêmes pour mettre un terme au conflit. Dans son analyse du problème, l'ApmJof adressa une critique subtile, mais forte à l'endroit de l'AJFO, dont elle remit en cause la « représentativité » :

Née de la consultation populaire et de la décision collective, l'Apmjof regroupe des mouvements de jeunes et des conseils étudiants qu'elle représente auprès des corps intermédiaires et des gouvernements, et à qui elle offre des services. La jeunesse en général a accès à ces services mais l'APMJOF ne représente que les mouvements qui l'ont mandatée. Née en 1949, l'Association de la jeunesse franco-ontarienne a vu le jour grâce à l'intervention de l'Association-mère des organismes francoontariens, soit l'Association canadienne-française de l'Ontario. L'AJFo veut répondre aux besoins de la jeunesse, regroupée ou non, par l'entremise de ses services. Que dire alors de la représentativité de l'AJfo ? Est-il possible de représenter « la jeunesse franco-ontarienne $»^{203}$ ?

Le message des auteurs du « livre blanc » était clair : l'APMJOF et l'AJFo étaient des créatures tout à fait différentes. L'une était née de la « consultation populaire » et de la « décision collective », elle était le fruit de la démocratie, de la volonté des jeunes de se responsabiliser et de participer activement et pleinement à leur propre épanouissement personnel. L'autre n'avait jamais été plébiscitée par la jeunesse franco-ontarienne elle-même, elle n'était guère plus, en définitive, que l'excroissance d'une association d'adultes, l'AcFéo en l'occurrence. La raison d'être et la légitimité des deux mouvements puisaient à des sources entièrement différentes, voire incompatibles. Il n'était pas difficile de deviner lequel des deux organismes était le plus « représentatif » aux yeux des auteurs du « livre blanc ». Contrairement à l'AJFO, l'APMJOF n'avait pas, non plus, l'ambition démesurée de regrouper tous les jeunes Franco-Ontariens de la province. Elle chapeautait, cependant, l'ensemble de leurs associations, tant au niveau provincial qu'au niveau régional. Ses «membres» étaient donc clairement identifiables. L'AJfo pouvait-elle en dire

203. Ibid., p. 2. 
autant? Qui représentait-elle, au juste, avec à peine une poignée de cercles locaux disséminés dans la province ? La jeunesse la boudait et les mouvements de jeunes s'étaient largement affiliés à l'APMJOF. Au nom de qui prétendait-elle parler ? La question de la « représentativité », de poursuivre les auteurs du document, était sans aucun doute le «nœud» du problème, auquel venait s'ajouter celui des divergences idéologiques, « puisque tout porteparole a[vait] un contenu à faire valoir ». Les deux associations étaient à couteaux tirés sur un grand nombre de questions, $\mathrm{y}$ compris le mode de légitimation dont elles se réclamaient, la question de la francisation de l'Université d'Ottawa, leur position respective face à l'ACFO, etc. «La prise de position de l'une entraîne le désaccord de l'autre ", déplora-t-on. La situation était devenue intolérable et risquait de les empêcher d'honorer leur contrat avec la jeunesse.

En réalité, le problème du dédoublement des services offerts à la jeunesse n'était que secondaire, le prétexte d'un affrontement plus large. Ces services étant de même nature, on pouvait facilement remédier au problème en les fusionnant, tout en permettant à chacun des organismes de conserver son autonomie. Cependant, il eût fallu, et c'est là où le bât blessait, qu'ils se partageassent clairement la tâche de représenter la jeunesse. La solution de l'ApMJOF était pour le moins audacieuse : en plus de fusionner leurs « services » culturels respectifs, elle proposa que l'AJFo limitât son champ d'intervention aux « travailleurs » et aux « professionnels » et qu'elle lui cédât, en échange, celui des étudiants ! L'ApMjof présenta sa proposition sous des dehors purement pragmatiques : «L'optique ou l'idéologie de l'un ou de l'autre n'entrerait pas en conflit et les besoins de tous les jeunes seraient comblés ${ }^{204}$. » Il était fort possible, cependant, qu'elle eût cherché à faire sa chasse gardée du milieu étudiant, celui qui fournissait aux mouvements de jeunesse leurs membres les plus actifs et les plus engagés. Que 1'AJFo eût accepté de limiter ses interventions auprès des travailleurs après avoir plutôt visé,

204. Ibid., p. 4. 
historiquement, à former la future élite dirigeante de la nation canadienne-française en Ontario aurait constitué, pour le reste, un revirement étonnant !

Le Conseil provincial de l'APMJOF fit parvenir le « livre blanc » à l'AJFo en même temps qu'une invitation à en débattre avec lui le 26 avril. Le procès-verbal de cette réunion importante est malheureusement introuvable, mais Franc-Jeu rapporta qu'on s'était entendu pour mettre sur pied un « comité de coordination » afin d'étudier les possibilités de rapprochement entre les deux mouvements ${ }^{205}$. L'esprit de collaboration qui avait régné, de toute évidence, lors de cette rencontre les conduisit à entreprendre un premier projet commun, soit l'organisation de la tournée du chansonnier Pierre Calvé en Ontario. L'AJfo et l'APMJof s'engagèrent aussi à tenir leur congrès respectif simultanément à Ottawa en octobre 1970. Ce double rassemblement représente véritablement un point tournant dans l'histoire des mouvements de jeunesse franco-ontariens. En effet, les quelque 400 jeunes qui y participèrent jetèrent les bases - encore une fois ! - d'un nouvel organisme qu'ils baptisèrent «Direction-Jeunesse » (DJ) ${ }^{206}$. Il s'agissait, selon Franc-Jeu, d'un geste de «solidarité » et la « manifestation d'un nouveau dynamisme chez [sic] la jeunesse de l'Ontario français ${ }^{207} »$. La tribune éditoriale de cette livraison du journal, qui revenait normalement à Jacques de Courville Nicol, fut occupée, cette fois, par nul autre que le rédacteur en chef du Droit, Marcel Gingras, celui-là même que de Courville Nicol avait pourfendu six mois plus tôt. La satisfaction et le bonheur de Gingras devant autant de « générosité », d' «abnégation », de « réalisme », de « sérieux », de « volonté » et d' « altruisme » étaient, de toute évidence, sans bornes ${ }^{208}$. Direction-Jeunesse

205. « Comité de coordination APMJOF - AJFo », Franc-Jeu, mai-juin 1970, p. 3, CRCCF, FAJFO, C9/36/8. L'APMJOF fut représentée, à ce comité, par Jean-François Aubé, Alain Picard et David Welch, tandis que l'AJfo y délégua Gérard Lévesque, JeanSerge Quesnel et Pierre Séguin.

206. «Une seule "Direction jeunesse" », Franc-Jeu, septembre-octobre 1970, p. 1, CRCCF, FAJFO, C9/36/8.

207. Ibid., p. 1.

208. Marcel Gingras, « Se tenir debout ! », Franc-Jeu, septembre-octobre 1970, p. 2, CRCCF, FaJfo, C9/36/8. 
n'était, pour l'instant, qu'un bureau intérimaire regroupant cinq délégués de chacune des deux associations qui l'avaient constitué209. L'AJfo et l'APMJOF conservaient chacune leur secrétariat général et continueraient de faire leurs propres demandes de financement auprès des gouvernements, mais DJ était habilitée à nommer un responsable de leurs «services » culturels désormais fusionnés. Le nouvel organisme avait aussi le mandat de rédiger de nouveaux statuts et d'organiser un véritable congrès de fondation en octobre 1971. Dans sa livraison de novembre-décembre 1970, Franc-Jeu publia, en guise d'éditorial, une caricature dans laquelle figuraient un homme et une femme censés représenter l'AJFo et l'APMJOF et se livrant à une étreinte on ne peut plus lascive... ${ }^{210}$ La jeunesse francoontarienne était entrée de plain-pied dans la révolution sexuelle, mais la lune de miel entre ses deux porte-parole provinciaux seraitelle suivie d'une union véritablement durable?

Il ne s'agissait, en réalité, que d'un mariage de raison aux fondements plus que fragiles. Dans le Franc-Jeu de janvier-février 1971, l'un des représentants de l'AJfo à Direction-Jeunesse, Pierre Ouellette, annonça avec fracas qu'il démissionnait de son poste ${ }^{211}$. Dans une longue lettre remplie d'amertume, il dénonça l'APMJOF qu'il accusa de travailler sans relâche à l'anéantissement des services culturels de l'AJFo et d'avoir imposé à cette dernière une dette colossale que ses propres subventions gouvernementales faramineuses n'avaient pas réussi à éponger. Le comble, c'était que l'ancien secrétaire général de l'ApmJof, Paul-François Sylvestre, était passé à l'emploi de la Direction de l'action socioculturelle du Secrétariat d'État, l'un des principaux bailleurs

209. Pour l'AJfo : Luc Charron (Ottawa), Pierre Séguin (Ottawa), Donald Poliquin (Ottawa), Pierre Ouellette (Ottawa) et Yvan Chénier (Timmins) ; pour l'APMJof : JeanPaul Aubé (Toronto), Jean-François Aubé (Toronto), Diane Gibeault (Crysler), Louise Tanguay (Hearst), Lucien Gava (Windsor). Voir "Une seule "Direction jeunesse" ", Franc-Jeu, septembre-octobre 1970, p. 1, CRCCF, FAJFO, C9/36/8.

210. « En guise d'éditorial [caricature de Guy Désilets] », Franc-Jeu, novembredécembre 1970, p. 2, CRCCF, FAJFO, C9/36/8.

211. Pierre Ouellette, «Pierre Ouellette démissionne de "Direction-Jeunesse"... Et pour causes ! », Franc-Jeu, janvier-février 1971, p. 1, 4, CRCCF, FAJFO, C9/36/8. 
de fonds de l'Association, sans jamais avoir renié ses anciennes allégeances. Cela revenait à « mettre la jeunesse à la merci d'un individu qui, par ses antécédents, ne $\mathrm{p}$ [ouvai]t être complètement objectif $^{212} »$. Mais il y avait pire encore : l'APMJOF aurait poussé l'odieux jusqu'à noyauter le congrès d'octobre 1970 ! L'un des cinq représentants de 1'AJfo à DJ, Yvan Chénier, n'était rien d'autre, d'après Pierre Ouellette, qu'une taupe ! Franc-Jeu publia, dans le même numéro, des lettres du président de l'ApMJOF, JeanFrançois Aubé, à plusieurs participants au congrès, dont Chénier, les remerciant du rôle « ingrat » qu'ils avaient joué dans le « coup d'État » qui avait permis la création de Direction-Jeunesse (lettres dont il n'a pas été possible, précisons-le, de vérifier l'authenticité).

Tout cela était absolument invraisemblable et exigeait que l'on prît les grands moyens pour rétablir une situation normale. Même l'éditrice de Franc-Jeu, Colette Langlois, menaça de quitter son poste à la suite des pressions exercées par l'APMJOF pour retirer le journal du contrôle de l'AJfo et le confier à DirectionJeunesse $^{213}$. Ouellette et Langlois reçurent de nombreuses lettres d'appui au lendemain de la parution de cette livraison de Franc$\mathrm{Jeu}$, sans compter une pétition comportant la signature d'une trentaine de jeunes de Vankleek-Hill soutenant 1'AJfo et demandant qu'elle fût réactivée ${ }^{214}$. Ces appuis persuadèrent Langlois de demeurer en poste, en fin de compte, mais elle revint à la charge contre l'APMJOF qu' elle accusa d'avoir censuré et saisi la dernière livraison de Franc-Jeu, celle dans laquelle Pierre Ouellette avait publié son article assassin. « La démocratie, écrivitelle, est actuellement faussée en Ontario par les nombreuses manigances de certains jeunes qui ne représentent pas l'ensemble de notre jeunesse ${ }^{215}$. » Dans le même numéro, le trésorier de DJ, Bernard Bonneville, annonça à son tour qu'il démissionnait en

212. Ibid.

213. Colette Langlois, « J'en ai plein mon casque ! », Franc-Jeu, janvier-février 1971, p. 2, CRCCF, FAJFO, C9/36/8.

214. Voir la correspondance de l'AJfo : CRCCF, FAJFO, C9/19/7.

215. Colette Langlois, « Direction-Jeunesse ou ... Direction-manipulation », avrilmai 1971, p. 2, CRCCF, FAJFO, C9/36/8 
raison des nombreuses irrégularités qu'il avait constatées dans les états financiers de l'organisme ${ }^{216}$. La pugnacité de l'organe de l'AJfo n'avait jamais été aussi grande. Cette livraison de FrancJeu n'en serait pas moins la dernière...

Direction-Jeunesse ne demeura pas impassible devant la fronde de sa rivale. Au printemps 1971, peu de temps après la dernière parution de Franc-Jeu, elle rédigea un document d'une dizaine de pages pour tenter de remettre les pendules à l'heure et de se défendre contre les accusations sérieuses qu'avait portées contre elle l'AJFO ${ }^{217}$. Ces dernières étaient entièrement dépourvues de fondements, expliqua-t-elle, et découlaient d'une volonté explicite de semer la zizanie au sein de la jeunesse. À l'AJfo comme à l'APMJOF, l'autorité suprême demeurait les émissaires que chacune des associations avait délégués à Direction-Jeunesse. Les initiatives que prenait cette dernière étaient donc parfaitement légitimes et consensuelles, aucune de ses deux composantes n'étant en mesure de prendre le dessus sur l'autre. Du demeurait unie et, malgré les coups bas et malhonnêtes qu'elle avait dû encaisser depuis sa fondation, plus solidaire que jamais. En ce qui avait trait à la question financière, elle avait eu recours aux services d'un vérificateur qui devait démontrer sous peu qu'il n'y avait eu aucune anomalie dans l'utilisation des subventions gouvernementales. Somme toute, Du (et l'APMJOF) était innocente des crimes qu'on avait voulu lui imputer ${ }^{218}$.

Le congrès d'octobre 1971 eut lieu comme prévu. Il n'attira, cependant, que 70 jeunes environ, ce qui ne représentait qu'une fraction du nombre total de délégués ayant participé au congrès conjoint de $1970^{219}$. Cela suffit tout de même pour que DirectionJeunesse vînt au monde officiellement. Elle ne s'éteindrait qu'au

216. Bernard Bonneville, « Démission du trésorier de Direction-Jeunesse », Franc-Jeu, avril-mai 1971, p. 1, CRCCF, FAJFO, C9/36/8.

217. «Direction-Jeunesse a six mois d'existence », [printemps 1971], CRCCF, Fonds Direction-Jeunesse, C54/1/11.

218. Il est intéressant de souligner, cependant, que Dı ne répondit pas directement aux accusations de noyautage que Pierre Ouellette avait portées contre l'APMJOF.

219. « Congrès 1971 [de Direction-Jeunesse, 8 au 11 octobre 1971]. Liste des congressistes », CRCCF, FAJFO, C9/42/7. 
bout de trois décennies de labeur, mais l'AJFO, pour sa part, avait déjà rendu l'âme. Une page de l'histoire de la jeunesse francoontarienne venait d'être tournée.

\section{Conclusion}

Dans une brève étude de ce qu'il appelle la « métamorphose » de la communauté franco-ontarienne de 1960 à 1985, Fernan Carrière écrit que les jeunes, à partir des années 1970, avaient « en général tendance à se méfier des discours nationalistes des dirigeants des organisations traditionnelles », et qu'ils « soupçonn[aient] dans ces discours des accents de nostalgie pour une époque révolue, que non seulement des jeunes mais aussi de larges secteurs de la population ne voul[ai]ent pas recréer ${ }^{220} \gg$. En effet, les mouvements de jeunesse franco-ontariens avaient connu, depuis le début des années 1960, une évolution substantielle, tant sur le plan de l'organisation que sur celui des représentations identitaires. Il ne faut pas sous-estimer le rôle qu'ont pu jouer les gouvernements, fédéral et provincial, dans cette évolution. À la fin des années 1960, alors que l'État-providence poursuivait inlassablement son expansion et que les fonctionnaires s'étaient convertis au principe de l'animation socioculturelle, les gouvernements, devenus de véritables vaches à lait pour les associations « de langue officielle », seraient en mesure, en revanche, d'exercer sur elles un ascendant certain. L'intervention des fonctionnaires et de la classe politique ontarienne dans le conflit qui sévissait entre l'AJFO et l'APMJOF, intervention qui contraignit les deux mouvements à lancer DJ, en fournit un exemple éloquent ${ }^{221}$.

220. Fernan Carrière, « La Métamorphose de la communauté franco-ontarienne, 1960-1985», op. cit., p. 324.

221. Certains chercheurs se sont interrogés sur le bien-fondé d'une telle dépendance vis-à-vis des gouvernements. Selon Daniel Savas, l'intervention financière du gouvernement fédéral auprès de la Fédération des Franco-Colombiens eut tendance à professionnaliser et à "fonctionnariser», en quelque sorte, les dirigeants de la communauté, qui tireraient désormais leur légitimité non plus d'un contact « organique » avec les Franco-Colombiens eux-mêmes, mais plutôt de leurs rapports avec l'État. Voir Daniel Savas, « L'Iimpact des politiques d'aide du Secrétariat d'État sur l'évolution 
Cependant, le rôle des gouvernements ne suffit pas à expliquer l'ampleur de l'évolution des mouvements de jeunesse francoontariens, que l'on peut imputer à la profonde mutation culturelle que vivait le monde occidental durant les années 1960. L'idéologie de participation en viendrait à balayer le nationalisme traditionaliste du Canada français au nom d'un idéal égalitariste et anti-élitiste. Le mandat initial de l'AJFo, qui avait été de former la future élite dirigeante de la nation canadienne-française en Ontario, serait de plus en plus perçu comme un archaïsme, le vestige d'une époque révolue qui ne correspondait plus aux valeurs démocratiques et individualistes auxquelles adhéraient les jeunes du baby boom à la fin de la période. L'AJfo, comme nous l'avons vu, avait elle-même pris des distances de plus en plus grandes vis-à-vis de ses anciennes convictions nationalistes et élitistes, mais à l'APMJOF, le rejet du traditionalisme canadienfrançais serait à peu près total. Si la référence nationale traditionaliste du Canada français était fondée sur la thèse de la création providentielle des nations et sur la conviction qu'il existait un ordre hiérarchique dans le monde, la mutation des années 1960 contribua à l'abandon d'une conception encore théocentrique de l'univers au profit d'une conception pleinement anthropocentrique. La jeunesse franco-ontarienne participa activement à la mise au rancart de l'identité canadienne-française, qui n'était pas, du moins en ce qui la concernait, que le résultat de la québécisation du discours nationaliste dans la « vieille province ».

Notre étude comporte cependant des limites. Si nous avons tenté de démontrer que les mouvements de jeunesse, durant les années 1960, définissaient de moins en moins l'identité des Franco-Ontariens en rapport avec la référence nationale canadienne-française, voire en rapport avec l'idée même de nation,

Voir Daniel Savas, « L'Impact des politiques d'aide du Secrétariat d'État sur l'évolution financière de la Fédération des Franco-Colombiens », dans Monique Bournot-Trites (dir.), Les Outils de la francophonie, Saint-Boniface, Centre d'études francocanadiennes de l'Ouest, 1988, p. 11-54; voir aussi Gratien Allaire, « De l'Église à l'État : le financement des organismes des francophones de l'Ouest, 1945-1970 », dans Jean Lafontant (dir.), L'État et les minorités, Saint-Boniface, Éditions du Blé et Presses universitaires de Saint-Boniface, 1993, p. 229-245. 
il n'est pas impossible, toutefois, que leurs revendications politiques aient continué de s'inspirer, jusqu'à un certain point, d'une logique nationaliste. Dès le début de la décennie, l'AJfo, comme nous avons pu le constater, s'était mise à concevoir l'identité « personnelle » du jeune Franco-Ontarien comme s'inscrivant dans un contexte « social », manifestement ontarien ou canadien, et de moins en moins " national », c'est-à-dire canadien-français. L'APMJof, quelques années plus tard, semblait toutefois avoir la fibre nationaliste un peu plus forte, comme en témoignaient ses prises de position sur la loi 63 au Québec et sur l'idée de franciser l'Université d'Ottawa, de même que l'importance qu'elle accordait à la langue et à la culture dans l'expérience collective des peuples. Une étude du discours et des activités de Direction-Jeunesse après 1971 nous permettrait de jauger ce qui devait subsister, dans l'esprit de ses dirigeants, de la logique nationale, de la volonté de continuer de «faire société222 » en Ontario français. Il n'en demeure pas moins, cependant, que les mouvements de jeunesse de la fin des années 1960 cessèrent de mobiliser explicitement la référence nationale canadienne-française, qu'ils associeraient désormais au traditionalisme et à l'élitisme. Force est de constater, dans le même ordre d'idées, que l'évacuation de la référence canadiennefrançaise donna lieu à un grand flou en ce qui avait trait à la définition de l'identité des jeunes Franco-Ontariens, pour peu que soient exclues les considérations plutôt vagues que l'on fit sur l'importance de la langue et de la culture dans la vie des « peuples ». Le phénomène n'avait cependant rien de surprenant. Contrairement à l'AJFo qui, au moment de sa fondation, avait voulu inculquer aux jeunes Franco-Ontariens une conception précise de l'identité canadienne-française, les mouvements de jeunesse de la fin des années 1960, qui ne juraient que par la « participation », étaient plutôt d'avis qu'il revenait au jeune de définir lui-même sa propre identité, que tout modèle imposé « d'en

222. Joseph-Yvon Thériault, Faire société. Société civile et espaces francophones, Sudbury, Prise de parole, 2007, 383 p. 
haut » eût été élitiste et illégitime (ce qui revenait au même, dans leur esprit).

Plutôt que de conduire les jeunes à réfléchir, par le truchement de l'étude, à la condition « nationale » des Franco-Ontariens, les mouvements de jeunesse de la fin des années 1960 voyaient leur rôle comme pourvoyeurs de « services » culturels. Plutôt que de former la future " élite dirigeante " de la nation canadiennefrançaise en Ontario, ils chercheraient à « représenter » la masse des jeunes et à favoriser leur «participation » à leur développement culturel personnel. Entre 1960 et 1971, c'est tout un monde qui bascula, celui de la tradition nationale canadienne-française. 